Editorial

\title{
Acknowledgment to Reviewers of Energies in 2020
}

\section{Energies Editorial Office}

Citation: Energies Editorial Office. Acknowledgment to Reviewers of Energies in 2020. Energies 2021, 14, 574. https://doi.org/10.3390/en1403 0574

Published: 23 January 2021

Publisher's Note: MDPI stays neutral with regard to jurisdictional claims in published maps and institutional affiliations.

\section{(c) (i)}

Copyright: (c) 2021 by the author. Licensee MDPI, Basel, Switzerland. This article is an open access article distributed under the terms and conditions of the Creative Commons Attribution (CC BY) license (http://creativecommons.org/licenses /by/4.0/).

MDPI AG, St. Alban-Anlage 66, 4052 Basel, Switzerland

Peer review is the driving force of journal development, and reviewers are gatekeepers who ensure that Energies maintains its standards for the high quality of its published papers. Thanks to the cooperation of our reviewers, in 2020, the median time to first decision was 17 days and the median time to publication was 39 days. The editors would like to express their sincere gratitude to the following reviewers for their precious time and dedication, regardless of whether the papers were finally published:

Aaheim, Asbjørn

Aaltonen, Jussi

Abad-Segura, Emilio

Abaide, Alzenira Da Rosa

Abarghani, Arash

Abastante, Francesca

Abba, Muhammad

Abbas, Ahmad

Abbas, Mehrabidavoodabadi

Abbasi, Qammer H.

Abbasi, Reza

Abbaspour Tamijani, Ali

Abbassi Monjezi, Alireza

Abbey, Samuel Jonah

Abbood, Sahar

Abdallah, Shaaban

Abdalrahman, Ahmed

Abdel Aleem, Shady H. E.

Abdelaziza, Sherif L.

Abdelhamed, Waleed

Abdelmaksoud, Ahmed

Abdel-Nasser, Mohamed

Abdelrahem, Mohamed

Abdelsalam, Mahmoud

Abdeltawab, Hussein M.

Abdrabou, Atef

Abdulagatov, Ilmutdin M.

Abdul-Aziz, Ali

Abdullah, Nur Azam

Abdur, Rahim

Abe, Rikiya

Abed, Suhail Najem

Abedinia, Oveis

Abeendranath, Abhinav Kalathiparambil

Abejón, Ricardo
Abella, Pura Alfonso

Åberg, Magnus

Abgottspon, Hubert

Abhishta, Abhishta

Abo-Al-Ez, Khaled M.

Abolude, Akintayo Temiloluwa

Abramchuk, Mykola

Abramek, Karol

Abramović, Borna

Abrishambaf, Omid

Abronzini, Umberto

Abu Ghazaleh, Haitham

Abudy, Menachem

Abuhussein, Ahmed

Accardo, Grazia

Accetta, Angelo

Acharya, Anirudh Budnar

Acharya, Bishnu

Achchaq, Fouzia

Achiaga Menor, Beatriz

Achim, Cristina

Achmus, Martin

Aciu, Claudiu

Aciu, Lia E.

Acquah, Moses Amoasi

Acquah-Andoh, Elijah

Acree Jr., William E.

Acznik, Ilona

Adachi, Yoshiaki

Adam, Antonis

Adam, George K.

Adam, Grain

Adam, Mario

Adamczyk, Arkadiusz

Adamczyk, Janusz 
Adamišin, Peter

Adamko, Peter

Adamkowski, Adam Tadeusz

Adamovský, Daniel

Adamski, Andrzej

Adamski, Mariusz Robert

Adamu-Lema, Fikru

Adan-Mas, Alberto

Adda, Mehdi

Addona, Fabio

Adegbohun, Feyijimi

Adegboye, Mutiu Adesina

Adege, Abebe Belay

Adekunle, Timothy O.

Adel, Amr

Aderinto, Tunde

Adhikari, Rajendra Singh

Adhikari, Ram C.

Adochiei, Felix

Adriana, Tokar

Aeberhard, Urs

Aelenei, Laura

Afa Michael, Inikuro

Afenyo, Mawuli

Afgan, Imran

Afghan, Syeda Adila

Aga, Alemu O.

Agana, Norbert

Agartan, Lutfi

Agathokleous, Rafaela

Agaton, Casper Boongaling

Agee, Philip

Agelin-Chaab, Martin

Aggarwal, Geetika

Aghaei, Mohammadreza

Aghazada, Sadig

Agonafir, Mesfin Belayneh

Ágoston, Katalin

Agresti, Filippo

Aguey-Zinsou, Kondo-Francois

Aguilar-Meléndez, Armando

Aguilera, Miguel Ángel Egido

Aguirre, Aitor Saenz

Agundis Tinajero, Gibran David

Agustin, Dominique

Ahac, Saša

Aharon, Ilan

Ahlström, Peter

Ahlswede, Erik

Ahmad, Muhammad Sajjad

Ahmad, Shafiq
Ahmad, Tanveer

Ahmadi, Esmaeil

Ahmadi, Maryam

Ahmadi, Mikaeel

Ahmadi, Mohammadali

Ahmadi, Seyed Farzad

Ahmadiahangar, Roya

Ahmadvand, Seyedsaeid

Ahmadyar, Ahmad Shabir

Ahmed, Abdullahi

Ahmed, Ahfaz

Ahmed, Fayez-Shakil

Ahmed, Mohamed

Ahmed, Sheikh

Ahmmad, Bashir

Ahn, Byung Tae

Ahn, Hyung Keun

Ahn, Jin-woo

Ahn, Joon

Ahn, Kyubok

Ahn, Seongho

Ahn, Seon-Ju

Ahokangas, Petri

Ahsan, Md Mominul

Ahsani, Vahid

Ahualli, Silvia

Aiello, Giuseppe

Aiello, Orazio

Aitkenhead, Matt

Aitkulov, Almas

Aivazidou, Eirini

Ajanovic, Amela

Ajib, Salman

Akan, Cigdem

Akander, Jan

Akanji, Lateef

Akbari Garakani, Mohammad

Akhmetzyanova, Uliana

Akhtar, Hussain

Akhtar, Saqib

Akhtarmanesh, Saman

Akhundjanov, Sherzod

Aki, Masahiko

Akiba, Etsuo

Akitsu, Takashiro

Akitsu, Tetsuya

Akkari, Nissrine

Akkerman, Ido

Aksanli, Baris

Al Ameri, Ahmed

Al Qubeissi, Mansour 
Al Siyabi, Idris

Al Sumaiti, Ameena

Ala, Guido

Al-Abadi, Ali

Al-Aboosi, Fadhil

Alabrudziński, Sławomir

Al-Ahmad, Alaa Yassin

Alahmad, Mahmoud

Alahyari, Arman

Al-Ali, Abdulrahman

Alam, K. C. Amanul

Alam, Miah Md Ashraful

Alam, Mollah Rezaul

Alam, Morshed

Alamaniotis, Miltiadis

Alamaniotis, Miltos

Alameda-Hernandez, Enrique

Al-Ameri, Talib

Al-Anssari, Sarmad

Alarco, Jose

Alasali, Feras

Alashloo, Yaser

Alazab, Mamoun

Alazne, Gutierrez

Albano, Maurizio

Albarracin, Ricardo

Albatici, Rossano

Albers, Albert

Albert, Ryan J.

Alberti, Anthony

Alberti, Luigi

Alberto, José Miguel

Alberto, Veses Roda

Alboteanu, Laurentiu

Alcaraz, Lorena

Alcarria, Ramón

Alcaso, Adérito Neto

Alcázar-Ortega, Manuel

Aldega, Luca

Aldeman, Matthew R.

Aldieri, Luigi

Alduse, Bejoy

Aleixo, Ana Marta

Alejandra, Boto Álvarez

Alejo Eleuterio, Roberto

Aleksandrova, Mariya

Aleksandrowicz-Trzcińska, Marta

Alemayehu, Fisseha M.

Alepuz, Salvador

Aleroev, Temirkhan
Aleshin, Andrey

Aleshin, Sergey

Alessandri, Angelo

Alessandro, Sbrana

Alevras, Panagiotis

Alexander, Aaron S.

Alexandersen, Joe

Alexandra, Jason

Alexandri, Eleftheria

Alexandridis, Antonio T.

Alexandrova, Larissa

Alexandru-Bogdan, Tatomir

Alexiev, Kiril

Alfano, Brigida

Alfaro, José Luis

Alfonso Piña, William $\mathrm{H}$.

Alfonso, Gago-Calderón

Alfranca, Oscar

Alfredo, Testa

Al-Ghussain, Loiy

Algieri, Angelo

Alhamdani, Wasim

Alhasan, Ahmad

Alhasheem, Mohammed

Alho, André Romano

Ali Abbas Kazmi, Syed

Ali Ahmed, Awadelrahman

Mohamedelsadig

Ali Ansari, Sajid

Ali, Babkir

Ali, Haider

Ali, Hassan

Ali, Husni Rois

Ali, Muhammad

Ali, Muhammad Umair

Ali, Naser

Ali, Nazakat

Alibakhshikenari, Mohammad

Alibašić, Haris

Aliberti, Alessandro

Alimonti, Claudio

Alin, Bosioc

Alińska, Agnieszka

Alishah, Rasoul Shalchi

Aliyu, Aliyu

Aliyu, Musa

Alizadeh Kordabad, Reza

Alizadeh, Morteza

Aljohani, Tawfiq

Alkasrawi, Malek

Alkhalaf, Haitham 
Allahdadi, Mohammad Nabi

Allam, Zaheer

Allard, Bruno

Allard, François

Allen, Kenneth

Allison, Gordon

Allmark, Matthew

Alloisio, Isabella

Allouis, Christophe

Ally, Moonis Raza

Al-Madhhachi, Abdul-Sahib

Almadhor, Ahmad

Almeida, Aitor

Almeida, Anibal

Almeida, Rita

Almenárez, Florina

Almodovar-Melendo, Jose-Manuel

Almonacid, Boris

Al-Muqdadi, Sameh W.

Al-Musaed, Amjad

Almutairi, Ghzzai

Alnajdi, Othman

Alobaid, Falah

Al-Obaidi, Karam

Alonso Tristán, Cristina

Alonso, Alberto Rodríguez

Alonso, Alicia

Alonso, Alvaro Hernandez

Alonso, Daniel Fernández

Alonso, Julio San José

Alonso, Monica

Alonso, Serafín

Alonso-Fariñas, Bernabé

Alonso-Marroquín, Fernando

Alothman, Amani

Alothman, Zeid

Al-Saadi, Mohammed

Al-Sadoon, Mohammed

Al-Sallami, Waleed

Al-Shannaq, Refat

Alshayeb, Mohammed J.

Alskaif, Tarek

Altan, Hasim

Altarawneh, Mohammednoor

Altarejos-García, Luis

Aluha, James

Alvarado-Barrios, L.

Álvarez Pardiñas, Ángel

Álvarez, Ana

Álvarez, Eduardo

Álvarez, Federico
Alvarez, Jon

Álvarez, María S.

Álvarez, Paloma

Alvarez-Diaz, Marcos

Alves, Ana

Alves, Luís Manuel

Alwaeli, Mohamed

Aly, Hamed

Alzahrani, Ahmad

Alzoubi, Mahmoud

Amadio, Emanuele

Amado, Miguel

Amaechi, Chiemela Victor

Amalevičiūtè Volungè, Kristina

Amalu, Emeka H.

Amancio, Diego

Amanianpong, Prince Nana

Amanowicz, Łukasz

Amara, Yacine

Amaral, António Luís

Amarandei, George

Amarasiri, Mohan

Amaris, Hortensia

Amasyali, Kadir

Amatya, Reja

Amavilah, Voxi Heinrich

Ambrosetti, Matteo

Ambrosio-lazaro, Roberto Carlos

Amedeo, Amoresano

Ameen, Arman

Amenta, Carlo

Amer, Mohammed

Ameur, Khaled

Ameyaw, Ernest Effah

Amezquita-Sanchez, Juan Pablo

Amin, Mohamad Hassan

Amin, Sakib-Bin

Aminnaji, Morteza

Amirat, Yassine

Amirkhani, Mehdi

Amirlatifi, Amin

Amiryar, Mustafa

Amnache, Amrid

Amores, Marco

Amoretti, Michele

Amosu, Adewale

Ampomah, William

Amro, Moh'd M.

Amuakwa-Mensah, Franklin

An, Hyosung

An, Jae-Sung 
An, Kwangjin

$\mathrm{An}, \mathrm{Lu}$

Anagnostopoulou, Afroditi

Anand, Prashant

Anani, Nader

Ananthakrishnan, Soundaram Jeevarathinam Angelov, Borislav

Anastasiadis, Anestis G.

Anastasiu, Livia

Ancellin, Matthieu

Ancona, Maria Alessandra

Anđelković, Aleksandar

Andersen, Pål

Anderson, Ben

Anderson, Brian

Anderson, C. Lindsay

Anderson, Nathan

Anderson, William A.

Andersson, Bengt

Andersson, Maria

Ando Junior, Oswaldo Hideo

Ando, Takao

Andoga, Rudolf

Andrada, Pedro

Andrada, Pere

Andras, Andrei

Andras, Iosif

Andrea, Bisignano

Andrea, Veronika

Andreasen, Casper Schousboe

Andreasen, Rasmus

Andreev, Valeriy

Andrei, Andreia

Andrei, Horia

Andrei, Jean-Vasile

Andrei, Mariana

Andreić, Željko

Andreini, Antonio

Andrenacci, Natascia

Andreoli, Enrico

Andrews-Speed, Philip

Andriamanohiarisoamanana, Fetra

Andric, Jelena

Andrievsky, Boris

Andriollo, Mauro

Andronic, Luminita

Androvitsaneas, Vasilios P.

Andruszkiewicz, Jerzy

Andrychowicz, Mateusz

Andrzej Kolator, Bronisław

Andrzej, Michalski

Andrzejczyk, Rafał
Andrzejewski, Jacek

Andwari, Amin Mahmoudzadeh

Ang, Yee Sin

Angelotti, Adriana

Angeloudis, Athanasios

Angelova, Mina

Angelova, Radostina A.

Angers, Jean-François

Anghel, Dragos-Victor

Anghel, Elena

Angrecka, Sabina

Anguera, Jaume

Anido Rifón, Luis E.

Aniello, Gianmarco

Annamalai, Kalyan

Annesini, Maria Cristina

Annuk, Andres

Anquetin, Sandrine

Ansart, Renaud

Anseán, David

Ansorge, Cedrick

Antal, Marcel

Antaluca, Eduard

Antchev, Mihail

Antczak, Elzbieta

Antelmi, Matteo

Anthony, Edward

Anticoi, Hernan

Antón, Javier Muñoz

Anton, Sorin

Antonini, Alessandro

Antonini, Enrico

António Correia, José

Antonopoulos, Angelos

Antonopoulou, Georgia

Antonov, Liudmil

Antonovskaya, Galina

Antuñano, Aitor Ciarreta

Antunes, Carlos Henggeler

Antunes, Mário

Anuchin, Alecksey

Anukam, Anthony

Anvar, Amir M.

Anvari-Moghaddam, Amjad

Anwar, Mohammad

Anweiler, Stanisław

Anysz, Hubert

Aoki, Mutsumi

Apel, Derek

Apollo, Michal 
Apostoluk, Aleksandra (Alexandra)

Appelbaum, Joseph

Aprea, Ciro

Aprilia, Ernauli

Apse-apsitis, Peteris

Apsley, Judith M.

Aquino, Andrea

Arabzadeh, Vahid

Arad, Susana

Araki, Kenji

Aram, Farshid

Aramendia, Iñigo

Arampatzis, Ioannis

Aranda, Miguel

Arandjelovic, Ognjen

Arango-Miranda, Raúl

Aranha, Jose

Arantzazu Gomez, María

Araoz, Joseph A.

Arato, Elisabetta

Araújo, António

Araújo, Noelia

Araújo, Rui Esteves

Aravelli, Aparna

Arbabi, Hadi

Arbabi, Sepehr

Arbizzani, Catia

Arčabić, Vladimir

Arcese, Gabriella

Archetti, Renata

Arcia-Moret, Andrés

Arcos-Vargas, Angel

Ardakani, Omid

Ardelean, Mircea

Arefin, Shezan

Arellano, Marisol Manzano

Arena, Maurizio

Arenas, Eva

Arenas, Luis Fernando

Arendas, Peter

Arentsen, Maarten

Aresti, Lazaros

Ariaratnam, Samuel

Arias Trujillo, Juana

Arias, Borja

Ariga, Katsuhiko

Arima, Hirofumi

Arionfard, Hamid

Ariyur, Kartik B.

Armaselu, Bogdan

Armstrong, Andrea
Armstrong, Paul

Arnaltes Gómez, Santiago

Arnaoutakis, Georgios E.

Arnau, Carles B.

Arnaudov, Dimitar

Arnesano, Marco

Arnold, Bruce Baer

Arora, Divyesh R.

Arora, Shashank

Arpino, Fausto

Arrabito, Giuseppe Domenico

Arrasmith, William Wolfgang

Arrebola, Manuel

Arredondo, Francisco

Arregi, Beñat

Arrhenius, Karine

Arrinda, Amaia

Arrizabalaga, Eneko

Arroyo, Alberto

Arroyo, Oscar

Arsalis, Alexandros

Arsenio, Pedro

Arsenovic, Pavle

Arshad, Adeel

Arshad, Ali

Arsiccio, Andrea

Artale, Giovanni

Artal-Sevil, Jesús Sergio

Artemov, Mikhail

Artiukhina, Ekaterina

Aruga, Kentaka

Arul, Narayanasamy Sabari

Arumugam, Saravanakumar

Arunachalam, Prabhakarn

Arvanitis, Antonios

Arvanitis, Konstantinos

Aryal, Ashrant

Aryal, Nabin

Aryana, Saman

Arza-García, Marcos

Arzamendi, Gurutze

Arzoumanidis, Ioannis

Asadi, Amin

Asadi, Somayeh

Asadzadeh, Mohammad Zhian

Asai, Masayasu

Asamoah, Richmond

Asano, Kohta

Asawa, Takashi

Ascencio-Vásquez, Julián

Aschilean, Ioan 
Aschonitis, Vassilis

Ascoli, Alon

Aseeva, Anna

Asem, Pouyan

Asghar Heidari, Ali

Ashrafi, Amirmansoor

Ashrafi, Nariman

Ashrafur Rahman, S. M.

Ashtine, Masao

Ashuri, Maziar

Asif, Amir

Asif, Muhammad

Asim, Taimoor

Asimakopoulou, Eleni

Askari, Radwin

Aslansefat, Koorosh

Asllani, Besar

Asorey-Cacheda, Rafael

Asprou, Markos

Assadian, Francis

Assimonis, Stylianos D.

Assirelli, Alberto

Astaneh, Majid

Astiaso Garcia, Davide

Astolfi, Davide

Åstrand, Per-Olof

Astrouski, Ilya

Asuka, Jusen

Asumadu-Sarkodie, Samuel

Atam, Ercan

Atanase, Leonard

Atanasoae, Pavel

Ataollahi, Narges

Atkins, Anthony S.

Atkins, Martin

Atkinson, Seddon

Atla, Veerendra

Atsonios, Konstantinos

Attaianese, Ciro

Attili, Antonio

Attya, Ayman

Auer, Hans

Auger, François

Augustine, Sijo

Augusto, Andres

Aune, Vegard

Aung, Kendrick

Aurangzeb, Khursheed

Ausiello, Gigliola

AuYeung, Nick

Avci, Civan
Avenas, Yvan

Avendaño-Valencia, Luis David

Averbukh, Moshe

Averfalk, Helge

Avgouropoulos, George

Avila, Deivis

Ávila, José Pablo Chaves

Avolio, Roberto

Avramova, Maria

Awan, Usama

Awasthi, Mayanka

Awni, Rasha A.

Awolayo, Adedapo

Awoleke, Obadare

Awtoniuk, Michał

Awual, Md Rabiul

Axaopoulos, Petros J.

Axon, Stephen

Ayachit, Agasthya

Aydin, Burchan

Ayed, Samah Ben

Ayub, Ahmad Syahrir

Azar, Ahmad Taher

Azarova, Olga A.

Azevedo Guedes, André Luis

Azevedo, Afonso

Azevedo, Alcino

Azib, Toufik

Aziz, Asma

Aziz, Muhammad

Azizi, Aydin

Azizi, Mohsen

Azman, Samet

Azmat, Muhammad

Azpúrua, Marco A.

Azuma, Takashi

Azzolini, Damiano

Azzuni, Abdelrahman

Babalola, Micky A.

Babazadeh, Davood

Babiarz, Bożena

Babiloni, Adrián Mota

Baborska-Narozny, Magdalena

Bacanin, Nebojsa

Baccarani, Giorgio

Baccioli, Andrea

Bacco, Manlio

Baccoli, Roberto

Bacher, Jean-Philippe

Bach-Oller, Albert

Baczyński, Dariusz 
Badami, Marco

Bade, Mehar

Badea, Dorel

Badea, Gheorghe

Badenes, Borja

Badesa, Luis

Badescu, Gabriel

Badescu, Viorel

Badilescu, Simona

Badyda, Krzysztof

Bae, Dowon

Bae, Youngchul

Baek, Changryong

Baek, Donkyu

Baek, Gahyun

Baek, Jeihoon

Baek, Kwang-Hyun

Baek, Seung-Mook

Baek, Soo-Whang

Baena, Francisco Manuel

Baeyens, Jan

Baeza Moyano, David

Bagalkot, Nikhil

Bagdanavicius, Audrius

Bagdžiūnas, Gintautas

Baggio, Paolo

Baglivo, Cristina

Bagnato, Giuseppe

Baguley, Craig

Bahar, Mohammad

Bahat-Treidel, Eldad

Baheri, Ali

Bahmani, Hamed

Bahmanteymouri, Elham

Bahrami, Sina

Bahramipanah, Maryam

Bai, Attila

Bai, Feifei

Bai, Hua

Bai, Jinbo

Bai, Lei

Bai, Linquan

Baiardi, Donatella

Baidar, Binaya

Baig, Zubair

Baigmohammadi, Mohammadreza

Baimel, Dmitry

Baïri, Abderrahmane

Baiutti, Federico

Bajguz, Andrzej

Bajpai, Ankur
Bak, Yeongsu

Baker, Kyri A.

Baker, Nick

Baker, Thar

Bakhshian, Sahar

Bakierska, Monika

Bakke, Rune

Bakon, Tomasz

Bakos, Levente

Bakran, Mark

Balacco, Gabriella

Balachandran, Ramanarayanan

Balado Frias, Jesus

Balaguru, Raja

Balamurugan, Rathinam

Balan, Mugur C.

Balaras, Constantinos A.

Balas, Valentina E.

Balasingam, Balakumar

Balasingam, Suresh Kannan

Balasubramanian, Karthik

Balato, Marco

Balaz, Ivan

Balazsi, Csaba

Balcázar, Néstor

Baldean, Doru

Baldi, Simone

Baldick, Ross

Baldinelli, Arianna

Baldini, Luca

Baldini, Mario

Baldovín, Francisco López

Bałdowska-Witos, Patrycja

Balduzzi, Francesco

Baleta, Jakov

Baležentis, Tomas

Balibrea, José

Bálint, Erika

Balis, Nikolaos

Ball, Christopher Stephen

Ballarini, Ilaria

Ballesteros, Rosario

Balling, Niels

Balocco, Carla

Balogh, Péter

Baloi, Alexandru

Băloi, Felicia-Ionela

Balsalobre-Lorente, Daniel

Baltakys, Kęstutis

Baltazar, Juan-Carlos

Ban, Marko 
Banaś, Jan

Banaszak, Szymon

Banaszek, Jerzy

Banaszkiewicz, Mariusz

Banaszkiewicz, Tomasz

Banerjee, Amitava

Banerjee, Anil

Banerjee, Aparajita

Banerjee, Arindam

Bang, Inkyu

Bang, Sungsig

Bangga, Galih

Banguero, Edison

Banias, George

Banionis, Karolis

Bannov, Alexander

Bañón, Fermín

Bañón, Luis

Baños Navarro, Raúl

Bansah, Kenneth

Banu, Ioan-Viorel

Bao, Jia

Bao, Jiming

Bao, Yi

Baptista, José Manuel Ribeiro

Barabas, Sorin

Barakat, Mahmoud R.

Barakhtenko, Evgeny

Baran, Jolanta

Baranski, Mariusz

Baranwal, Rohit

Baranyai, Nóra

Barasinski, Anais

Barata, Filipe

Barata, Jorge

Barati, Masoud

Barati, Reza

Barbanti, Lorenzo

Barbara, Uliasz-Misiak

Barbarelli, Silvio

Barbaresi, Alberto

Barbato, Marco

Barbato, Maurizio

Barbera, Elvira

Barbera, Orazio

Barberes, Gabriel

Barbeş, Lucica

Barbieri, Riccardo

Barbón, Arsenio

Barbosa, Ricardo

Barbour, Edward
Bărbuţă-Mişu, Nicoleta

Barca, Emanuele

Barcellona, Simone

Barchi, Grazia

Barelli, Linda

Bares, Pau

Barez, Fred

Bargiotas, Dimitrios

Bari, Saiful

Barić, Tomislav

Bark, Chung Wung

Barkai, Allatchi Hassan

Barlik, Roman

Barltrop, Nigel

Barmada, Sami

Barmpatza, Alexandra C.

Barna Iantovics, Laszlo

Barnes, Frank S.

Barnes, Jake

Barnett, Allen

Barone, Giuseppe

Baronti, Federico

Baroutaji, Ahmad

Barpaga, Dushyant

Barradas, Filipe M.

Barrado-Rodrigo, Jose A.

Barragán, V. María

Barragán-Villarejo, Manuel

Barreca, Francesco

Barreras, Felix

Barreras, Jorge

Barrero, Federico

Barrero-Gil, Antonio

Barreto, Germilly

Barrios, Pedro Vergara

Barro, Christophe

Barron, Robert

Barry, Matthew

Barsi, Dario

Barta, Dalibor

Barth, Kurt L.

Bartkowiak, Tomasz

Bartlomiejczyk, Mikolaj

Bartniczak, Bartosz

Bartocci, Pietro

Bartoletti, Cipriano

Bartoli, Mattia

Bartolomeo, Antonio Di

Bartolucci, Lorenzo

Bartoňová, Lucie

Bartoszewicz, Jarosław 
Bartyś, Michał

Barukčić, Marinko

Baruque Zanon, Bruno

Barwicki, Jan

Barz, Cristian

Barz, Tilman

Basavarajappa, Manjunath

Basbas, Socrates

Bascompta, Marc

Basha, Omar

Bashian, Amir

Bašić, Josip

Bašić, Mateo

Basińska, Małgorzata

Basioli, Lovro

Basso, Daniele

Basso, Gianluigi Lo

Bastiaans, Rob

Bastida Domínguez, María

Bastidas, David M.

Bastola, Ebin

Bastos, Alvaro Furlani

Basu-Gupta, Sudeshna

Baszynski, Marcin

Batista, Maria João

Batmunkh, Munkhbayar

Batog, Jolanta

Batool, Munira

Batrakov, Alexander

Batrancea, Ioan

Battarra, Mattia

Battineni, Gopi

Battista, Federico

Battistel, Postdoc Alberto

Battisti, Alessandra

Batuecas, Esperanza

Bauer, Anton Johann

Bauer, Christian

Bauer, Jan (Czech Republic)

Bauer, Jan (Germany)

Bauer, Martin

Bauer, Pavol

Bauer, Stephen

Baum, Seth

Baumann, Marcus

Baumgarten, Martin

Bäumle, Roland

Baure, George

Bautista, Luis Fernando

Bauwens, Pieter

Bavasso, Irene
Bayati, Navid

Bayram, Islam Safak

Bayuk, Irina

Bazan, Peter

Bazbauers, Gatis

Bazilevskaya, Katya

Bazmohammadi, Najmeh

Bazooyar, Bahamin

Bazydło, Grzegorz

Bazzaz, Mohammad

Bazzi, Ahmad

Bazzo, Thiago

Beach, Thomas H.

Bean, Richard

Beath, Andrew

Beaudoin, Anthony

Beben, Damian

Bechhoefer, Eric

Beciu, Silviu

Beck, Hauke

Beck, Krzysztof

Becker, Maik

Beckers, Koenraad F.

Beckingham, Lauren E.

Beckmann, Volker

Bécsi, Tamás

Beddiar, Karim

Będkowski, Bartłomiej

Bednar, Slavomir

Bednarek, Karol

Bednarz, Lukasz

Bedon, Chiara

Bee, Elena

Beer, Martin

Beer, Martin D.

Beganovic, Nejra

Beghetto, Valentina

Begines Ruiz, Belén

Behrendt, Cezary

Behrens, Sam

Beidaghy Dizaji, Hossein

Beik, Omid

Beirão, Pedro

Bejaković, Predrag

Bejan, Adrian

Bejan, Iustinian

Belan, Marco

Belardini, Elisabetta

Beleiu, Horia Gheorghe

Belenguer, Enrique

Belgiorno, Giacomo 
Belhaj, Hadi

Beliatis, Michail J.

Belibassakis, Kostas

Beligiannis, Grigorios N.

Belke, Ansgar H.

Bell, Ian H.

Bell, John

Bell, Simon

Bella, Federico

Bella, Gino

Bellan, Diego

Bellanger, Gilbert

Belleudy, Cecile

Bellia, Laura

Bellini, Alberto

Bellos, Evangelos

Bellucci, Alessandro

Belman-Flores, Juan Manuel

Belmont, Mike

Belousov, Evgeny V.

Beltrán, Héctor

Beltrán, Juan

Belu, Nadia

Belu, Radian

Belussi, Lorenzo

Belvederesi, Chiara

Ben Elghali, Seifeddine

Beňa, Lubomír

Benassai, Guido

Benato, Alberto

Benbouzid, Mohamed

Beneda, Károly

Benedetti, Ilaria

Bengoa, Christophe

Benigni, Andrea

Benim, Ali Cemal

Benito, Inés García

Beniuga, Razvan

Bennamoun, Lyes

Bennewitz, John

Bennici, Simona

Beňová, Mariana

Benseman, Timothy

Bentsen, Niclas Scott

Benvenuto, Giovanni

Beraldi, Patrizia

Bercu, Ana-Maria

Bercu, Mioara

Berenblyum, Roman

Berenguel, Manuel

Berger, Matthias
Berggren, Björn

Bergillos, Rafael J.

Bergland, Olvar

Bergman, Anders

Bergot, Thierry

Berhausen, Sebastian

Berlowska, Joanna

Bernacki, Krzysztof

Bernard, Brian

Bernard, Geffroy

Bernard, Pauline

Bernardi, Bruno

Bernardino, Mariana

Bernardo, Luís

Bernaś, Marcin

Bernasconi, Giancarlo

Bernasowski, Mikołaj

Bernat, Jakub

Bernesson, Sven

Bernhoff, Hans

Berni, Rossella

Bernstein, David H.

Bernuy-Lopez, Carlos

Berrittella, Maria

Bertacchini, Alessandro

Bertaina, Sylvain

Bertelsen, Nis

Bertoldi, Paolo

Bertolini, Marina

Bertrand, Raquel García

Berzina, Kristina

Besagni, Giorgio

Beschkov, Venko

Bessac, Julie

Bessler, Sandford

Beszédes, Sándor

Betancur, Stefanía

Beu, Dorin

Bevilacqua, Carmelina

Beyabanaki, S. Amir Reza

Beyer, Hans Georg

Bezha, Minella

Bhaduri, Moinak

Bhagavathy, Sivapriya M.

Bhagia, Samarthya

Bhandari, Janarjan

Bhandari, Khagendra

Bhandari, Manohar Prasad

Bharadwaj, Vivek

Bharati, Guna R.

Bhatia, Divij 


\begin{tabular}{|c|c|}
\hline Bhatia, Shashi Kant & Birdwell, Justin \\
\hline Bhattacharya, Devanjan & Birke, Kai Peter \\
\hline Bhattacharya, Sumit & Birnie, Dunbar P. \\
\hline Bhattacharyya, Subhes C. & Birou, Iulian M. T. \\
\hline Bhattarai, Arjun & Birrell, Stewart \\
\hline Bhattarai, Susma & Bisello, Adriano \\
\hline Bhochhibhoya, Silu & Bishop, Wade \\
\hline Bhuiyan, Ehsan & Biswas, Soumava \\
\hline Bhuiyan, Md Al Masum & Biswas, Soumyajyoti \\
\hline Bhuiyan, Mohammad & Biswas, Sumana \\
\hline Bhuyan, Arupjyoti & Biswas, Swarup \\
\hline Biagetti, Giorgio & Bithas, Petros S. \\
\hline Białowiec, Andrzej & Bitoleanu, Alexandru \\
\hline Biały, Witold & Bizhani, Majid \\
\hline Bianchi, Alberto & Bizmark, Navid \\
\hline Bianchi, Giuseppe & Bizon, Katarzyna \\
\hline Bianchi, Massimo & Bizon, Nicu \\
\hline Bianchi, Valentina & Bjelić, Ana \\
\hline Bianchini, Alessandro & Bjørkevoll, Knut \\
\hline Bianco, Davide & Błachuta, Marian \\
\hline Bianco, Nicola & Black, Geoffrey \\
\hline Bibra, Mohit & Black, Leon \\
\hline Bica, Dorin & Blaha, Petr \\
\hline Bicek, Matej & Blair, Niebert \\
\hline Biel, Carmen & Blake, Lynsay \\
\hline Bielecki, Sławomir & Blakers, Andrew \\
\hline Bień, Andrzej & Blanchard, Richard \\
\hline Bieniek, Tomasz & Blanco, Jesús Maria \\
\hline Bienvenido-Huertas, David & Blanco, Juan \\
\hline Bierhoff, Michael & Blanco-Fernandez, Pilar \\
\hline Biernat, Krzysztof & Blandin, Gaetan \\
\hline Bifaretti, Stefano & Blanes, Jorge \\
\hline Bijaieh, Mehrzad M. & Blaschke, Paul \\
\hline Bila, Alberto & Błasiak, Przemysław \\
\hline Bilan, Yuriy & Blaszczuk, Artur \\
\hline Bilandžija, Nikola & Błażejczyk-Majka, Lucyna \\
\hline Bilas, Vedran & Błażejewski, Ryszard \\
\hline Bilbao, Julia & Blazevic, Zoran \\
\hline Bilík, Petr & Blažič, Boštjan \\
\hline Bilski, Piotr & Blecharz, Krzysztof \\
\hline Bimbela, Fernando & Blecich, Paolo \\
\hline Bin Tarek, Md Tawhid & Blekhman, David \\
\hline Biňasová, Vladimíra & Bless, Frédéric \\
\hline Binci, Daniele & Bliūdžius, Raimondas \\
\hline Bindzár, Peter & Bloemendal, Martin \\
\hline Biniak-Pieróg, Małgorzata & Blomberg, Sara \\
\hline Binkowski, Tomasz & Blondeau, Julien \\
\hline Binzhong, Zhou & Blum, Helcio \\
\hline Biondi, Elisabetta & Boada, Maria Jesus Lopez \\
\hline Birau, Ramona & Boansi, David \\
\hline Birbas, Michael & Boaro, Marta \\
\hline
\end{tabular}


Bobadilla, Luis F.

Boban, Luka

Bober, Peter

Bobi, Miguel Angel Sanz

Bobinger, Marco

Bocart, Fabian

Boccaletti, Chiara

Boccardo, Gianluca

Boccarusso, Luca

Bocchetta, Patrizia

Bocci, Enrico

Bocîi, Liviu Sevastian

Böckl, Benjamin

Bocklisch, Thilo

Boddapati, Loukya

Bode, Arndt

Bode, Christoph

Bode, Florin

Bodini, Nicola

Bodisco, Timothy

Bodkhe, Sampada

Bodo, Enrico

Boenzi, Francesco

Boer, Dieter

Bogacki, Jan

Bogdan Mihai, Niculescu

Bogdan, Paul

Bogdanovich, Valeriy I.

Bogdanowicz, Krzysztof Artur

Bogovalov, Sergey

Bogoyavlensky, Vasily

Bogusz, Piotr

Bohaienko, Vsevolod

Bohdal, Tadeusz

Bohinc, Klemen

Böhm, Benjamin

Böhm, Michał

Bojnec, Stefan

Bokalič, Matevž

Bokde, Neeraj

Bokel, Regina

Bokor, Balázs

Boldyryev, Stanislav

Bolgov, Viktor

Bollino, Carlo Andrea

Bolonio, David

Bombac, Andrej

Bombek, Gorazd

Bomberg, Mark

Bon, Volodymyr

Bonavolontà, Francesco
Bonduà, Stefano

Bone, Gary

Bonelli, Francesco

Bonfio, Claudia

Bonomo, Matteo

Bonomolo, Marina

Bontoux, Laurent

Booker, Edward

Bordet, Alexis

Borecki, Michal

Borg, Simon

Borge-Diez, David

Borges, Cruz E.

Borggaard, Jeff

Borisov Todorov, Ivan

Borodinas, Sergejus

Boronin, Sergei

Borovik, Igor

Borovinskaya, Ekaterina S.

Borowicz, Marcin

Borowski, Marek

Borowski, Sebastian

Borowski, Sylwester

Borozan, Djula

Borquist, Eric

Borri, Emiliano

Borrmann, Thomas

Borsukiewicz-Gozdur, Aleksandra

Bortolini, Lucia

Borugadda, Venu Babu

Borunda, Mónica

Borychowski, Michał

Borz, Stelian Alexandru

Bosich, Daniele

Bosioc, Ilie Alin

Bosisio, Alessandro

Bosma, Bret

Bosnar, Sanja

Bošnjaković, Mladen

Bossola, Filippo

Boswell, Ray

Both, Arend-Jan

Botor, Dariusz

Botzheim, Janos

Boucherif, Abderraouf

Boucheron, Romuald

Boudissa, Rabah

Boufadel, Michel

Bouguelia, Mohamed-Rafik

Bouhouras, Aggelos S.

Boulama, Kiari Goni 
Boulogeorgos, Alexandros-Apostolos A.

Bouloumpasis, Ioannis

Bounaceur, Roda

Bourikas, Leonidas

Bouris, Demetri

Bourtsalas, Athanasios

Bousbaine, Antonia

Boutayeb, Mohamed

Bouzid, Allal

Bouzourâa, Montassar Billeh

Bovo, Cristian

Bowman, Robert C.

Boxman, Raymond L.

Boy, Virginie

Boyer, Robert H. W.

Bozalakov, Dimitar

Bozhko, Yulia Yu

Bozorg, Mokhtar

Brachi, Paola

Bracinik, Peter

Bradac, Zdenek

Bragança, Luís

Bragg-Sitton, Shannon

Bragin, Mikhail

Braithwaite, Nicholas

Bramesfeld, Goetz

Brameshuber, Mario

Branca, Teresa Annunziata

Branch, Philip

Branchini, Lisa

Brandell, Daniel

Brandštetter, Pavel

Brandusoiu, Ionut Bogdan

Brasiello, Antonio

Bratu, Polidor

Bratuškins, Uğis

Bravi, Marco

Brazdil, James F.

Brcic, David

Brdulak, Anna

Breard, Arnaud

Breban, Stefan

Brecha, Robert J.

Brecl, Kristijan

Breen, Michael

Breesch, Hilde

Breitschopf, Barbara

Bremdal, Bernt Arild

Bremer, Jörg

Breńkacz, Łukasz

Brenna, Stefano
Brent, Alan

Breschi, Valentina

Bresciani, Etienne

Bressot, Christophe

Bretfeld, Mario

Bretón, Julia Griselda Cerón

Brezesinski, Torsten

Briat, Olivier

Brideau, Sebastien

Bridgwater, Anthony

Brillard, Alain

Brinkerhoff, Joshua

Briongos, Samira

Briševac, Zlatko

Briso, Cesar

Brito, Miguel Centeno

Brito, Moisés

Brito, Paulo

Brito, Paulo S. D.

Briz, Fernando

Brkić, Dejan

Brock, Philippa M.

Brodal, Eivind

Brodie, Graham

Brodnianska, Zuzana

Brodny, Jarosław

Brojboiu, Maria

Broniewicz, Elzbieta

Bronin, Dimitriy

Bronstein, Svetlana

Bronzaft, Arline

Brooks, Adria

Brooks, Robert

Broseta, Daniel

Bross, Lisa

Brown, Anthony

Brown, Kristen

Brown, Philip N.

Brown, Robert Keith

Brozyna, Jacek

Brtník, Bohumil

Brumerčík, František

Brun, Nicolas

Brunerová, Anna

Brunesi, Emanuele

Bruninx, Kenneth

Brunklaus, Gunther

Brunner, Samuel

Bruno, Roberto (University of Bologna, Italy)

Bruno, Roberto (University of Calabria, Italy)

Brunoro, Silvia 


\author{
Brus, Grzegorz \\ Brusca, Sebastian \\ Brutti, Sergio \\ Bruzzese, Claudio \\ Bryant, John \\ Bryant, Steven \\ Bryja, Leszek \\ Bryk, Maja \\ Brzezińska, Dorota \\ $\mathrm{Bu}$, Siqi \\ Bubach, Bailey \\ Bubnov, Alexey \\ Buccella, Concettina \\ Bucci, Giovanni \\ Bucci, Vittorio \\ Buccino, Mariano \\ Buchan, Andrew \\ Buchman, Attila \\ Buciakowski, Mariusz \\ Bučinskas, Vytautas \\ Bück, Andreas \\ Buckman, Jim \\ Bucko, Samuel \\ Bucovețchi, Olga Maria Cristina \\ Budaciu, Cristina \\ Budinski, Vedran \\ Budžaki, Sandra \\ Budzianowski, Wojciech \\ Bueno, Emilio J. \\ Bueso, María C. \\ Buffa, Simone \\ Bugała, Artur \\ Bui, Binh \\ Bui, Ngoc-Tam \\ Buick, James \\ Buijs, Wim \\ Buire, Jérôme \\ Buiu, Catalin \\ Bujok, Petr \\ Bujoreanu, Carmen \\ Bukhari, Syed Basit Ali \\ Bukowski, Przemyslaw \\ Buła, Dawid \\ Bulic, Neven \\ Bumiller, Gerd \\ Bunea, Andrea \\ Bungau, Constantin \\ Bungau, Simona \\ Bunster, Victor \\ Burchett, Bradley T. \\ Burduhos, Bogdan-Gabriel
}

Burdziakowski, Paweł

Bureika, Gintautas

Bureš, Vladimír

Bureva, Veselina

Burganos, Vasilis N.

Burgas, Llorenç

Burgio, Alessandro

Burlacu, Andrei

Burlakovs, Juris

Burlea-Schiopoiu, Adriana

Burlica, Radu

Burlikowski, Wojciech

Burman, Esfandiar

Burmester, Daniel

Burnete, Nicolae Vlad

Burnley, Stephen

Burns, Jonathan

Burugupally, Sindhu Preetham

Burzyński, Damian

Buschmann, Matthias H.

Busu, Cristian

Busu, Mihail

Butala, Vincenc

Butcher, Daniel

Buticchi, Giampaolo

Butkus, Mindaugas

Butrylo, Boguslaw

Butrymowicz, Dariusz

Buttay, Cyril

Butu, Alina

Bütün, Hür Ebuzer

Butusov, Denis

Butzlaff, Felix

Buzási, Attila

Buzatu, Andrei

Buzio, Marco

Buzna, Lubos

Byeon, Seongjoon

Byrne, Julie

Byrne, Paul

Byun, Sangjin

Byun, Yung-Cheol

Bzducha-Wróbel, Anna

Cabaleiro, David

Caballero, Damian

Caballero, Víctor

Caban, Jacek

Cabeza-Lainez, Jose M.

Cablé, Axel

Cabral Pinto, Marina

Cabrera Tobar, Ana 
Cacabelos, Antón

Cacuci, Dan

Cadelano, Gianluca

Cadenas, Erasmo

Čađenović, Rade

Cadez, Simon

Cadoux, Florent

Caesarendra, Wahyu

Caetano, Nídia

Caggiano, Antonio

Caglayan, Dilara Gülcin

Cagnano, Alessia

Cahoy, Dexter

Cai, Hanmin

Cai, Jianchao

Cai, Jihua

Cai, Mengmeng

Cai, $\mathrm{Wu}$

Cai, Yuhang

Caignaert, Guy

Cailean, Alin-Mihai

Cała, Marek

Calabrò, Paolo S.

Calabrò, Roberto

Calado, Maria Do Rosário Alves

Calamante, Massimo

Calamita, Giuseppe

Calandrini, Sara

Calatroni, Alberto

Calbó, Josep

Călbureanu, Mădălina

Caldeira, Laura

Caleiro, António

Caliciotti, Andrea

Calif, Rudy

Calin, Adrian Cantemir

Calle Cancho, Jesús

Callejo, Miguel Ángel Manso

Calvert, Kirby

Calvo Herrero, Luis Miguel

Calvo, Francisco

Calvo-Silvosa, Anxo

Calzaferri, Gion

Camacho, Eduardo F.

Camarinha-Matos, Luis M.

Camci, Cengiz

Camelia, Gabor

Camm, Joseph

Campagnolo, Filippo

Campello-Vicente, Hector

Campillo, Jose Miguel
Campisi, Tiziana

Campo, Celeste

Campo, Riccardo

Campos, Urso

Campu, Vasile

Canals, Vincent

Cañas, Ignacio

Cancelliere, Piergiacomo

Cancelo, Maite Teresa

Candamano, Sebastiano

Candelieri, Antonio

Canders, Wolf

Candra, Dodiek Ika

Candrea, Adina Nicoleta

Canepa, Edward

Canhoto, Paulo

Caniato, Marco

Canivell, Jacinto

Canizes, Bruno

Cannarile, Francesco

Cannavale, Alessandro

Cannistraro, Giuseppe

Cano-Espinosa, Carlos

Cano-Ortega, Antonio

Cansado, Pablo

Cansino, José M.

Cantero, Alvaro Serna

Cao, Bingyang

Cao, Bo

Cao, Jianyun

Cao, Liangzhi

Cao, Mengqiu

Cao, Tao

Cao, Wen-Ping

Cao, Yiding

Cao, Yuan

Cao, Yunqi

Capaldo, Luca

Capata, Roberto

Capezzali, Massimiliano

Capitani, Renzo

Capitman, John Amson

Capizzi, Giacomo

Capodaglio, Andrea G.

Capogni, Marco

Caporali, Stefano

Caporin, Massimiliano

Capote, Rene

Capozzoli, Alfonso

Cappai, Francesco

Cappuzzello, Francesco 
Capraro, Valerio

Capuano, Francesco

Capuano, Paolo

Capuder, Tomislav

Caputo, Fabio

Carabano, Rocio

Cárabe, Julio

Carabineiro, Sonia

Caraiani, Petre

Cara-Jiménez, Jorge

Carapeto, Ana

Carbone, Alessandra

Carbone, Lorenzo

Carboni, Gianluca

Carcadea, Elena

Cardaci, Alessio

Cardador-Martínez, Anaberta

Cardona, Fabio

Cardoso, José Roberto

Caricchi, Federico

Čarija, Zoran

Cariow, Aleksandr

Carleer, Robert

Carli, Raffaele

Carlo, Petrarca

Carlsson, Anders E.

Carlucci, Claudia

Carmen Carnero, María

Carmona, Josep

Carnì, Domenico

Carnieletto, Laura

Caro, Eduardo

Carole, Coufort

Carozza, Antonio

Carpenter, Adam T.

Carpentier-Postel, Samuel

Carpinelli, Guido

Carpita, Mauro

Car-Pusic, Diana

Carr, Stephen

Carratù, Marco

Carravetta, Armando

Carreño-Ortega, Angel

Carreon, Moises

Carretero, Claudio

Carrier, Marion

Carril, Jose Carbia

Carrillo, Alfonso J.

Carrión, Miguel

Carriveau, Rupp

Carta, Daniele
Carter, David A.

Cartland-Glover, Gregory

Caruana, Cedric

Carulli, Francesco

Caruso, Giulia

Caruso, Ugo

Carutasiu, Mihail-Bogdan

Carutasu, George

Caruzzo, Amaury

Carvalho, Carlos

Carvalho, José Beleza

Carvalho, Luisa M. H.

Carvalho, Mónica

Carvalho, Rosário

Casado, Alejandro

Casado-Belmonte, María Del Pilar

Casado-Coterillo, Clara

Casadonte, Dominick

Casale, Giuseppe Rocco

Casaleiro, João

Casasso, Alessandro

Cascardi, Alessio

Cascella, Giuseppe Leonardo

Cascone, Stefano

Caseiro Rocha, Alfredo Moreira

Caseiro, Luís

Casella, Patrizia

Casini, Marco

Casisi, Melchiorre

Casoli, Paolo

Casolino, Giovanni Mercurio

Cassarino, Tiziano Gallo

Cassetti, Gabriele

Castaing-Lasvignottes, Jean

Castaldo, Anna

Castañeda-Miranda, Alejandro

Castaño, Fernando

Castaño, Maria-Soledad

Castaño-Rosa, Raúl

Castedo, Ricardo

Casteleiro-Roca, Jose Luis

Castellani, Beatrice

Castellani, Francesco

Castellano, Nuria Novas

Castellanos, Sergio

Castelletto, Nicola

Castelli, Ivano E.

Castelli-Dezza, Francesco

Castelló, Ramon Costa

Castiglione, Teresa

Castilla, Roberto 
Castillo Olea, Cristian

Castillo, Ismael

Castillo, Tony

Castro Dominguez, Bernardo

Castro, Edison

Castro, Eulogio

Castro, Jesús

Castro, José Raúl

Castro, Pablo

Castro-García, M. P.

Castronuovo, Edgardo D.

Castro-Osma, José A.

Castro-Santos, Laura

Catalano, Agostino

Catalano, Antonio Pio

Catalin Daniel, Galatanu

Catalina-Lucia, Cocianu

Cataliotti, Antonio

Ćatipović, Ivan

Catita, Cristina

Catizzone, Enrico

Catrini, Pietro

Catry, Filipe

Cattanei, Andrea

Cattani, Luca

Cavaco Mendes, Mário José Gonçalves

Cavagnaro, Robert

Cavaliere, Giuseppe

Cavalieri, Salvatore

Cavallaro, Antonio

Cavallaro, Fausto

Cavallaro, Luca

Cavallo, Alberto

Cavazzini, Giovanna

Cavenaghi, Marcos

Cayeux, Eric

Cazacu, Emil

Cazan, Cristina

Cazzaniga, Noemi E.

Cebrucean, Dumitru

Cebula, Richard

Cecchini, Massimo

Cecconet, Daniele

Cecconi, Manuela

Ceci, Michelangelo

Cecílio, José

Cecotti, Marco

Cecrdle, Jiri

Cedano, Karla G.

Cedomir, Milosavlevic

Čegar, Saša
Ceglia, Francesca

Čekon, Miroslav

Celadyn, Magdalena

Celani, Alberto

Celik Girgin, Sinem

Celik, Ilke

Celikbilek, Ozden

Celli, Daniele

Celli, Gianni

Cen, Yinjie

Cenký, Matej

Centeno, Miguel Angel

Centobelli, Piera

Čeponis, Andrius

Ceran, Bartosz

Cerasi, Pierre Rolf

Cerciello, Francesca

Cerda, Emilio

Cerdeira, Fernando

Cereceda, Oihane

Čereška, Audrius

Ceresoli, Davide

Cerezo-Narváez, Alberto

Čermák, Jiří

Cernat, Mihai

Černeckienè, Jurgita

Cerone, Nadia

Cerretti, Giacomo

Cerri, Giovanni

Cerro-Prada, Elena

Cervi, Eric

Cesare Redondi, Alessandro Enrico

Cespi, Daniele

Cha, Ho-Young

Cha, Hyeongyun

Chaabane, Wassim

Chabane, Sofiane

Chaberek, Grazyna

Chaczykowski, Maciej

Chadha, Kanwal

Chae, Junjae

Chai, Wei

Chai, Zhenhua

Chakirov, Roustiam

Chakrabarti, Amartya

Chakrabarti, Sambuddha

Chakraborty, Debapriya

Chakraborty, Manali

Chakraborty, Nirjhor

Chakraborty, Uday

Chakrapani, Sunil 
Chalikias, Miltiadis

Chalkias, Dimitris A.

Chalupka, Karolina

Chamakos, Nikolaos

Chambers, Jonathan

Chamorro Vera, Harold R.

Chamorro, César R.

Champier, Daniel

Chan, Chien Aun

Chan, Fan Liang

Chan, Steve

Chan, Sunney

Chan, Ying-Chieh

Chand, Shyam

Chandra Rao, Varikuti Purna

Chandrahalim, Hengky

Chandrala, Lakshmana

Chandramouli, Kulshreshtha

Chandrasekhar, Arunkumar

Chang, Byungik

Chang, Cheng-Hsun-Tony

Chang, Chia-Lin

Chang, Choong-Koo

Chang, Daejun

Chang, Der-Wen

Chang, Han

Chang, Hsueh-Hsien

Chang, Hsueh-Sheng

Chang, Jui-Cheng

Chang, Kuo-Jen

Chang, Liann-Be

Chang, Pearl Chun

Chang, Ping

Chang, Rui

Chang, Seongju

Chang, Shuchih Ernest

Chang, Tsangyao

Chang, Victor

Chang, Ya-Ju

Chang, Yao-Feng

Chang, Yeong-Hwa

Chang, Yi-Kuo

Chang, Yuan-Chih

Chang, Yu-Chia

Chang, Yuhua

Chao, Ru-Min

Chaouch, Mounir

Chapman, Andrew John

Chappidi, Chandrakanth

Charalampakos, Vassilios

Charalampopoulos, Ioannis
Charisiou, Nikolaos

Charles, Komboigo

Charpentier, Jean-Claude

Charvat, Karel

Charvát, Pavel

Charvátová, Hana

Chase, Alison

Chassiakos, Athanasios

Château, Pierre-Alexandre

Chatterjee, Atanu

Chatterjee, Tanmoy

Chaturvedi, Tanmay

Chatzakis, John

Chatzitheodoridis, Fotios

Chau, Kwok-Wing

Chau, Tat Kei

Chaubey, Saurabh

Chaudhari, Ashvinkumar

Chaudhary, Sanjay K.

Chaudhry, Hassam

Chaudhry, Muhammad Umar

Chauhan, Aneesh

Chauhan, Kapil

Chava, Rama Krishna

Chaves Ávila, José Pablo

Chavez-Angel, Emigdio

Chayaamor-Heil, Natasha

Chazallon, Bertrand

Che, Yuzhang

Cheah, Lynette

Cheba, Katarzyna

Cheluszka, Piotr

Chen, Bang-Fuh

Chen, Bolin

Chen, Chen

Chen, Cheng-I

Chen, Cheng-Wei

Chen, Cheng-Yi

Chen, Chiachung

Chen, Chien-Sheng

Chen, Chih-Chiang

Chen, Chin-Chao

Chen, Chin-Shan

Chen, Chun

Chen, Daniel

Chen, Han-Shen

Chen, Haoyong

Chen, Hongtian

Chen, Hsuan-Chu

Chen, Hung-Cheng

Chen, Jiann-Jong 
Chen, Jian-Zhang

Chen, Jia-Shiun

Chen, Jiefu

Chen, Jim Shih-Jiun

Chen, Jinhua

Chen, Jun

Chen, Jyh-Jian

Chen, Kun-Yung

Chen, Lea-der

Chen, Leo

Chen, Li

Chen, Liang-Bi

Chen, Liang-Yu

Chen, Lin

Chen, Lingen

Chen, Lung-Chien

Chen, Ming-Hung

Chen, Pao Chi

Chen, Peirong

Chen, Peng

Chen, Po-Tuan

Chen, Ruei-Tang

Chen, Shang-Yuan

Chen, Shengen

Chen, Shin-Ju

Chen, Shujian

Chen, Tao

Chen, Tao-Hsing

Chen, Ti

Chen, Tsung-Chia

Chen, Wei-Bo

Chen, Weiqiang

Chen, Wenfu

Chen, $\mathrm{Xi}$

Chen, Xiao

Chen, Yan (Hawaii Natural Energy

Institute, USA)

Chen, Yan (Pacific Northwest National

Laboratory, USA)

Chen, Yang

Chen, Yi-Chung

Chen, Yi-Yung

Chen, Yong-Song

Chen, Yuan-Ho

Chen, Yukai

Chen, Yung-Chung

Chen, Zengshun

Chen, Zhi-Gang

Chen, Zhixi

Cheng, Chun-An

Cheng, Eric Ka-Wai
Cheng, Hsu-Yung

Cheng, Huanyu

Cheng, Jui-Hung

Cheng, Ka Wai Eric

Cheng, Liang

Cheng, Pei

Cheng, Ruiqi

Cheng, Tao

Cheng, Weiying

Cheng, Xiaodong

Cheng, Yang-Tse

Cheng, Yu-Huei

Cheng, Yuk Shing

Cheong, Jae Hak

Cheraghian, Goshtasp

Cherednichenko, Oleksandr

Cheremisin, Alexey

Cherian, Chinchu

Chernoray, Valery

Chernyak, Sergei

Chernyi, Sergei

Cheskis, Dima

Cheung, Ocean

Cheval, Sorin

Chevallier, Julien

Cheverda, Vladimir A.

Chew, Nick Guan Pin

Chi, Ting

Chiacchio, Ferdinando

Chiampo, Fulvia

Chiandone, Massimiliano

Chianese, Simeone

Chiang, Chen-Yu

Chiappini, Daniele

Chiatti, Giancarlo

Chiba, Kazuhisa

Chibiryaev, Andrey

Chicco, Jessica Maria

Chicea, Dan

Chicet, Daniela

Chiementin, Xavier

Chien, Nguyen Ba

Chiesa, Giacomo

Chilet, Salvador Seguí

Chin, Alan H.

Chin, Cheng Siong

Chin, Jit Kai

Chinnici, Marta

Chiodo, Elio 
Chioncel, Cristian Paul

Chiriac, Radu

Chirkov, Denis

Chirumamilla, Manohar

Chisholm, William A.

Chitale, Kedar

Chitnis, Mona

Chiu, Huang-Jen

Chiu, Lai Fong

Chiu, Linus Y. S.

Chiu, Wei-Yu

Chiu, Yong-Hu

Chivelet, Nuria Martín

Chmiel, Jarosław

Chmielewski, Adrian

Chmielewski, Maciej

Chmielewski, Tomasz

Chng, Samuel

Cho, Chungyeon

Cho, Dae Won

Cho, Guyoung

Cho, Gyu-Jung

Cho, Hwanhee

Cho, Il-Hyeong

Cho, In Sun

Cho, Jung Sang

Cho, Kyu Taek

Cho, Seokwon

Cho, Soo-hwan

Cho, Young-Hoon

Chodakowska, Ewa

Chodkowska-Miszczuk, Justyna

Choe, Yoonsik

Choi, Bong Jun

Choi, Byungcho

Choi, Dae-Hyun

Choi, Daiwon

Choi, Donguk

Choi, Hojong

Choi, Jong Min

Choi, Jungwon

Choi, Jun-Ho

Choi, Kwang Hwan

Choi, Kyunghwan

Choi, Minsuk

Choi, Myong Yong

Choi, Myoung Shik

Choi, Nag Jung

Choi, Sang-Il

Choi, Seok Min

Choi, Sungyong
Choi, Sungyun

Choi, Suyong

Choi, Ui-Min

Choi, Wonik

Choi, Wooyeol

Choi, Woo-Young

Choi, Yeong-Jun

Choi, Yong Chan

Choi, Yongho

Choi, Yong-Seok

Choi, Yosoon

Choi, Youngjin

Choiński, Dariusz

Chojnacki, Andrzej Lukas

Chojnacki, Jerzy

Cholewa, Tomasz

Chomát, Miroslav

Chondrogiannis, Stamatios

Chong, Adrian

Chong, Heap-Yih (John)

Choo, Kyosung

Choon-Man, Jang

Choroszucho, Agnieszka

Chorowski, Maciej

Chou, Cheng-Tung

Chou, Eric Ting-Kuei

Chou, Jung Chuan

Choubar, Mohammad Akbari

Choudhary, Hemant

Chouhan, Shailesh Singh

Chow, David

Chow, Wan-ki

Chowdhury, Dhiman

Chowdhury, Jaideep

Chowdhury, Satyajit

Chowdhury, Towhid Hossain

Chrenko, Daniela

Chríbik, Andrej

Christanis, Kimon

Christen, Thomas

Christian, Ostertag-Henning

Christidis, Panayotis

Christodoulides, Paul

Christodoulou, Christos A.

Christoforidis, Georgios

Christophe, Sauvey

Christos, Kordulis

Chruszcz-Lipska, Katarzyna

Chrysoulas, Christos

Chrzan, Krystian Leonard

Chrzan, Piotr 
Chrzanowski, Łukasz

$\mathrm{Chu}$, Hongqing

Chu, Hung-Chi

Chua, Beelee

Chuang, Ching-Chun

Chuang, Ming-Chin

Chuang, Po-Ya Abel

Chudy-Laskowska, Katarzyna

Chuffart, Thomas

Chung, Haegeun

Chung, Ilyong

Chung, Jae Dong

Chung, Jaewoo

Chung, Kung-Ming

Chung, Sheng-Heng

Chung, Yao-Liang

Chung, Yi-Nung

Chung, Young Mo

Chuvilin, Evgeny

Chybowski, Leszek

Ciabattoni, Lucio

Ciaburro, Giuseppe

Ciancaleoni, Gianluca

Ciancetta, Fabrizio

Ciani, Adriano

Ciani, Lorenzo

Čibinskienè, Akvilè

Cican, Grigore

Cicconi, Paolo

Cicea, Claudiu

Cicone, Antonio

Cienfuegos-Suárez, Pablo

Ciesielski, Wojciech

Cignini, Fabio

Cinà, Lucio

Cinefra, Maria

Cinocca, Andrea

Cinteza, Otilia Ludmila

Ciobotaru, Mihai

Cioccolanti, Luca

Cioncolini, Andrea

Ciontea, Catalin-Iosif

Ciornei, Irina

Ciortan, Sorin

Cipek, Mihael

Cipollone, Roberto

Cirà, Andrea

Ciri, Umberto

Ciric, Rade M.

Cirimele, Vincenzo

Cirlugea, Mihaela
Cirstea, Marcian

Cirstea, Stefan

Cirtoaje, Cristina

Cisek, Piotr

Cislaghi, Alessio

Ciszewski, Mateusz

Citroni, Rocco

Ciuciu, Ioana

Ciuonzo, Domenico

Ciupitu, Liviu

Ciurzyńska, Agnieszka

Clairand, Jean-Michel

Clancy, Joy

Clar, Christoph

Clark, David

Clark, Thomas A.

Clarkson, Peter

Claudio, Gianfranco

Clausen, Niels-Erik

Clausen, Philip

Clauss, John

Clavero, Maria

Clavier, Nicolas

Cleary, Patricia

Clematis, Davide

Clemens, Markus

Clements, Adam

Climent, Javier

Climente-Alarcon, Vicente

Cloete, Schalk

Clough, Peter

Cobianu, Cornel

Coca, Eugen

Coccia, Gianluca

Cocho-Bermejo, Ana

Cochrane, Logan

Coclanis, Peter

Coclite, Alessandro

Cocolios, Thomas Elias

Codecasa, Lorenzo

Coderoni, Silvia

Codetta Raiteri, Daniele

Coe, Ryan

Coelho, Leandro Dos Santos

Coelho, Luis

Coelho, Paulo

Coelho, Pedro J.

Cogato, Alessia

Cohen, Boiko

Čoko, Duje

Colafranceschi, Stefano 
Colangelo, Gianpiero

Colantoni, Andrea

Colbertaldo, Paolo

Colclough, Shane

Coldea, Teodora Emilia

Cole, Wesley

Coletti, Chiara

Coli, Massimo

Colla, Valentina

Collado, José Villar

Collares-Pereira, Manuel

Colli, Alejandro Nicolás

Collins, John

Colom, Xavier

Coloma, Juan Francisco

Colorado-Garrido, Dario

Colson, Gregory

Colter, Peter

Coman, Mihaela Denisa

Coman, Vasile

Combes, Francois

Combrinck, Madeleine

Conceição, Eusébio Z. E.

Concli, Franco

Conejo, Antonio

Conesa, Juan A.

Conflitti, Paolo

Congedo, Paolo Maria

Congestri, Roberta

Coni, Mauro

Čonka, Zsolt

Connolly, James P.

Consolini, Giuseppe

Consorti, Lorenzo

Constable, Edwin

Constantin Neagu, Bogdan

Constantin, Gabriel-Alexandru

Constantina, Ciprian

Constantinescu, Radu

Constantinescu-Dobra, Anca

Conte, Francesco

Contestabile, Pasquale

Conti, Massimo

Contini, Daniele

Continillo, Gaetano

Cook, Ann

Cook-Chennault, Kimberly

Copping, Andrea E.

Coppo, Massimiliano

Coppola, Gennaro

Corazza, Maria Vittoria
Córcoles-Tendero, Juan I.

Corcoran, Peter

Cordes, Florian

Cordiner, Stefano

Čorejová, Tatiana

Corey, Gabriel

Corgnale, Claudio

Corizzo, Roberto

Corkish, Richard

Cornaro, Cristina

Cornet, Iris

Coronas, Alberto

Čorović, Selma

Corrado, Rindone

Corrado, Vincenzo

Corral Bobadilla, Marina

Corrales-Serrano, Mario

Corrao, Rossella

Correa, Lina Brand

Corrêa, Tomás P.

Correia, Sandra F. H.

Corsini, Alessandro

Cortellessa, Gino

Cortes, Domingo

Corti, Fabio

Cortiços, Nuno D.

Cortignani, Raffaele

Corvacho, Helena

Cosanti, Barbara

Cosentino, Valentina

Cosimbescu, Lelia

Cosoiu, Costin Ioan

Cossu, Marco

Costa, Aniko

Costa, Carlos Miguel

Costa, Fiammetta

Costa, Francisco

Costa, Paulo Moisés

Costa, Susana

Costa, Vítor Antõnio Ferreira

Costa-Castelló, Ramon

Costall, Aaron W.

Costamagna, Paola

Costanzo, Paola

Costanzo, Vincenzo

Costea, Monica

Costinas, Sorina

Cotas, Carla

Cote, Gérard

Cotfas, Petru

Cotorcea, Alexandru 
Cottis, Panayotis

Cottone, Giulio

Couenne, Francoise

Coufal, Oldřich

Coughlin, Michael

Coulter, John

Couraud, Benoit

Coutelieris, Frank A.

Coutinho, Joana T.

Couto, António

Couto, Luis D.

Couto, Paulo

Covaci, Stefan

Coz, Alberto

Cozzolino, Raffaello

Craciun, Eduard-Marius

Craciunescu, Aurelian

Craciunescu, Teddy

Craig, Michael T.

Crandall, Dustin

Cravero, Carlo

Crawley, Jenny

Creager, Stephen E.

Crebier, Jean-Christophe

Crepeau, John

Crespi Llorens, Damian

Crespo Cabillo, Isabel

Crespo, Nuno

Cressman, John R.

Cretan, Remus

Cretu, Mihaela

Creux, Patrice

Crijns-Graus, Wina

Crippa, Paolo

Crisan, Gloria Cerasela

Crisp, Ryan W.

Crispino, Gaetano

Criste, Adina

Cristea, Ciprian

Cristian, Dumitru

Cristiani, Cinzia

Cristian-Ioan, Leahu

Cristina Rada, Elena

Cristina, Rodriguez

Croce, Pietro

Crocker, Mark

Cronin, Leroy

Croonenbroeck, Carsten

Cross, Jeffrey S.

Crossland, Andrew F.

Crotti, Gabriella
Crovetti, Paolo Stefano

Crozier, Constance

Cruz, Sérgio

Cruz-Romero, Pedro

Csaba, Fogarassy

Csaba, Toth

Csáky, Imre

Csiszár, Csaba

Csonka, Bálint

Cuadrado, David Gonzalez

Cuadras, Angel

Cubukcuoglu, Beste

Cucchiella, Federica

Cucinotta, Filippo

Cucinotta, Giuseppe

Cuculić, Aleksandar

Cucuzzella, Michele

Cude, Brenda

Cuenot, Bénédicte

Cuevas, Fermin

Cuevas-Martínez, Juan Carlos

Cugliari, Jairo

Cui, Minming

Cui, Qingyu

Cui, Ryna Yiyun

Cui, Yi

Cui, Yuyan

Cukrov, Neven

Cultrera, Matteo

Cumming, Adam S.

Cumming, Douglas

Cunningham, Rebecca

Cupiał, Michał

Curado, António

Curbelo, Jezabel

Curcio, Mariangela

Curling, Simon F.

Curtis, Robin

Curto, Domenico

Cusenza, Maria Anna

Cusidó, Jordi

Cvetković, Marko

Cvetkovic, Milos

Cvitic, Ivan

Ćwieląg-Piasecka, Irmina

Cyklis, Piotr

Cyran, Katarzyna

Cyrus, Václav

Czajka, Krzysztof Marek

Czapla, Tomasz

Czarnecki, Lech 
Czarnota, Joanna

Czarnota, Robert

Czekała, Wojciech

Czermański, Ernest

Czerwinski, Robert

Czop, Monika

Czujko, Tomasz

Czumbil, Levente

Czyż, Zbigniew

D'Accadia, Massimo

D'Acci, Luca

D'Acierno, Luca

D'Adamo, Idiano

D'Agostino, Fabio

D'Amato, Egidio

D'Ambrosio, Francesca Romana

D'Ambrosio, Stefano

D'Andrea, Antonio

D'Andreagiovanni, Fabio

D'arpino, Matilde

D'Auria, Francesco

D'Errico, Gianluca

D'Eusanio, Manuela

D’Urso, Sebastiano

Da Costa Branco, Paulo Jose

Da Costa Neves, Carlos Guilherme

Da Costa, Cesar

Da Costa, Ricardo M. F.

Da Graça, Guilherme Carrilho

Da Rosa, Leonardo Machado

Da Silva, Liliane Severino

Da Silva, Luís Pinto

Da Silva, Márcio José

Da Silva, Patrícia Pereira

Da Silva, Paula F.

Da Silva, Wilton

Da Silveira Petruci, João Flávio

Dabija, Ana-Maria

Dabija, Dan-Cristian

Dabrowska, Dominika

Dabrowska, Magdalena

Dąbrowski, Wojciech

Dach, Jacek

Dadashzadeh, Nima

Dadic, Martin

Dadzie, John

Dafnis, Spiros D.

Dagnon, Soleya

Dagoumas, Athanasios

Dahle, Sebastian

Dähler, Fabian
Dahmen, Nicolaus

Dai, Baiqian

Dai, Ching-Liang

Dai, Jian

Dai, Jiejian

Dai, Jing

Dakka, Sam M.

Dal Borgo, Mattia

Daliento, Santolo

Dalla Chiara, Bruno

Dalla Santa, Giorgia

Dama, Murali

Damacharla, Praveen

Damasevicius, Robertas

DÁmato, Roberto

Damiani, Leonardo

Damiano, Alfonso

Damma, Devaiah

Damodaran, Krishnan

Damodaran, Murali

Dan, Horatiu

Daneshazarian, Reza

Dani, Samir

Daniel, Lyrian

Daniele, Elia

Danielewicz, Jan

Danielewski, Marek

Danielis, Peter

Danielis, Romeo

Danilevičius, Algimantas

Danish, Mir Sayed Shah

Danko, Matúš

Dann, Sandie

Dannier, Adolfo

Danso-Boateng, Eric

Danza, Ludovico

Danzer, Michael

Daprà, Irene

Darab, Pompei-Cosmin

Darani, Shadi

Darby, Sarah J.

Darbyshire, Robyn

Daripa, Prabir

Darko, Amos

Darmon, Ruxandra

Daroch, Maurycy

Darvini, Giovanna

Das, Biplab

Das, Narottam

Das, Prodip

Dascalescu, Lucian 
Dashti, Hossein

Dashtian, Hassan

Dashtipour, Kia

Daskalopoulou, Aspasia

Daskiran, Cosan

Daunys, Gintautas

Davarikia, Hamzeh

Davaritouchaee, Maryam

Davarpanah, Afshin

David, Christin

Davidescu, Adriana Ana Maria

Davidson, Josh

Davidy, Alon

Davies, Ian

Davino, Daniele

Davis, Maggie R.

Davis, Mary

Davis, R. Benjamin

Davis, Ryan D.

Davoudi, Masoume

Dawid, Leszek

Dawidowski, Wojciech

Dawson, Grant

Dawson, James

De Blasio, Gabriele S.

De Carlo, Filippo

De Carne, Giovanni

De Caro, Fabrizio

De Carvalho, Rogerio Atem

De Cássia Oliveira Gomes, Fátima

De Castro Rodríguez, M ${ }^{\mathrm{a}}$ Teresa

De Cauwer, Cedric

De Crescenzo, Carmen

De Diego Poza, Luis Francisco

De Dikdan, Licia Diana Pietrosemoli

De Dios, José Carlos

De Donato, Giulio

De Falco, Domenico

De Falco, Pasquale

De Faria, Luciano Caruggi

De Francisco, Antônio Carlos

De Giorgi, Maria Grazia

De Gooyert, Vincent

De Gregorio Hurtado, Sonia

De Haart, L. G. J.

De Iuliis, Silvana

De Jesus Romero-Troncoso, Rene

De Jonge, Nadieh

De La Calle, Alberto

De La Casa Higueras, Juan

De La Franier, Brian
De La Morena, Joaquin

De La Nieta, Agustín Sanchez

De La O Serna, José Antonio

De La Vega, David

De Lorenzo, Giuseppe

De Luca, Luigi

De Luca, Pierantonio

De Mingo López, Luis Fernando

De Monte, Filippo

De Nicola, Antonio

De Oliveira, Helena Maria Santos

De Oliveira, Helinando Pequeno

De Oliveira, Leise Kelli

De Oliveira, Regiane Alves

De Oliveira-De Jesus, Paulo

De Pablo Valenciano, Jaime

De Pablo, Santiago

De Paepe, Michel

De Paepe, Ward

De Palma, Pietro

De Paola, Pierfrancesco

De Paoli, Marco

De Pascale, Andrea

De Paulis, Francesco

De Ridder, Fjo

De Rubeis, Tullio

De Santiago, Juan

De Santiago-Perez, J. Jesús

De Santis, Michele

De Sequeira, César Augusto Correia

De Simone, Marco Claudio

De Simón-Martín, Miguel

De Sousa Figueira Barata, Filipe André

De Sousa Oliveira, Gisela Marta Teixeira

De Sousa, Fernando Rangel

De Souza E Silva, Rogério Diogne

De Tommasi, Luciano

De Tuglie, Enrico

De Vero, Jeffrey

De Vita, Mariangela

De Vries, Gerdien

De Vries, Jan

De, Arijit

De, Shuvodeep

Deac, Teodora

Deaconu, Adrian

Deaconu, Ioan-Dragos

Deaconu, Sorin Ioan

Deakin, Elizabeth A.

Deangeli, Chiara

Debnath, Kumar Biswajit 
Deboucha, Walid

Dębowski, Marcin

Decaix, Jean

Dechouniotis, Dimitris

Deeba, Shohana Rahman

Defilippis, Paolo

Defoe, Jeff

Degano, Michele

Degiuli, Nastia

Deguchi, Yoshihiro

Dehabadi, Leila

Dehdarian, Amin

Dehghani, Hamidreza

Dehghani-Sanij, Alireza

Dei, Michele

Deilami, Sara

Deja, Mariusz

Dejaco, Mario Claudio

Dejhalla, Roko

Del Ama Gonzalo, Fernando

Del Hierro, Isabel

Del Olmo Larrañaga, Jon

Del Rio Oliveira, Santiago

Del Rio, Gabriel

Del Sarto, Daniele

Delač, Boris

De-Las-Heras, Ana

Delcea, Camelia

Delfs, Jens-Olaf

Delgado Galván, Xitlali

Delgado Mendinueta, José Manuel

Delgado Sobrino, Daynier Rolando

Delgado, Antonio

Delgado, Carmen

Delgado, Fernando

Delgado, João M. P. Q.

Delgado, Sergio Nogales

Delgado-Bonal, Alfonso

Delgado-Sanchez, Jose-Maria

Delichatsios, Michael A.

Deligant, Michael

Delinchant, Benoit

Dell'Agli, Gianfranco

Dell' Anna, Federico

Dell' Anna, Maria Michela

Dell'Isola, Marco

Dell'Ovo, Marta

Della Torre, Augusto

Delle Monache, Maria Laura

Delle Piane, Massimo

Delnavaz, Aidin
Delponte, Ilaria

Delzendeh, Elham

Dematteis, Erika

Dembińska, Izabela

Demidova, Galina L.

Demir, Sumeyra

Demirci, Umit B.

Demirhan, Haydar

Demková, Lenka

Demuren, Ayodeji

Denac, Matjaz

Denai, Mouloud

Dénarié, Alice

Deng, Mingcong

Deng, Tong

Deng, Xiong

Denholm, Paul

Deniau, Virginie

Denisiewicz, Arkadiusz

Dennington, Simon

Denzer, Anthony S.

Deppman, Airton

Dergiades, Theologos

Deriszadeh, Ali

Derugo, Piotr

Desgranges, Lionel

Deshmukh, Suhrid

Deskos, Georgios

Desmaële, Denis

Desmet, Bernard

Desmet, Jan

Desmulliez, Marc

Despalatovic, Marin

Dessante, Philippe

Dettori, Marco

Deur, Josko

Deveci, Muhammet

Deveerasetty, Kranthi Kumar

Devlin, John Paul

Devlin, Mark T.

Dey, Shreya

Dhakal, Chandra

Dhaliwal, Gurpinder Singh

Dhariwal, Rohit

Dhaundiyal, Alok

Dhimish, Mahmoud

Di Battista, Davide

Di Blasio, Gabriele

Di Capua, Francesco

Di Cara, Dario

Di Cocco, Vittorio 
Di Corato, Luca

Di Fraia, Simona

Di Giacomo, Gabriele

Di Ilio, Giovanni

Di Leonardo, Lino

Di Lillo, Paolo

Di Marcoberardino, Gioele

Di Masi, Sabrina

Di Nardo, Armando

Di Naso, Vincenzo

Di Natale, Francesco

Di Noia, Luigi Pio

Di Nunzio, Luca

Di Pace, Roberta

Di Piazza, Maria Carmela

Di Pretoro, Alessandro

Di Rienzo, Roberto

Di Sarli, Valeria

Di Schio, Eugenia Rossi

Di Silvestre, Maria Luisa

Di Turi, Silvia

Di Turo, Francesca

Diab, Youssef

Diaconita, Vlad

Diaconu, Bogdan

Diaconu, Daniel Constantin

Diakakis, Michalis

Diallo, Demba

Diamantopoulou, Vasiliki

Dias Pereira, Luisa

Dias, João

Dias, Marta

Dias, Rui

Diaz Blanco, Manuel Jesus

Diaz Gomez, César

Díaz Pérez, Francisco Javier

Diaz, Jose

Díaz, Juan José Vinagre

Díaz, Katia María Argüelles

Diaz, Sarai

Díaz-Blanco, Ignacio

Díaz-Dorado, Eloy

Díaz-Madroñero, Manuel

Diaz-Roldan, Carmen

Diaz-Sarachaga, Jose Manuel

Diaz-Somoano, Mercedes

Dicarlo, Anthony A.

Dick, Erik

Didaskalou, Eleni

Diederichsen, Kyle

Diego, Isidro
Diémoz, Henri

Dien, Bruce

Diesendorf, Mark

Diez, José M.

Diez, José Ramón

Díez, Maria Antonia

Díez-Jiménez, Efrén

Digiesi, Salvatore

Dijkstra, Atze

Dilonardo, Elena

Dima, Stefan-Ovidiu

Dimakis, Nicholas

Dimeas, Aris

Dimino, Ignazio

Dimitrakopoulos, Georgios

Dimitrijevic, Aleksandra

Dimitrijevic, Branka

Dimitrios, Bikas

Dimitrios, Dimitrakis

Dimitrios, Kogias

Dimitriou, Dimitrios

Dimitrov, Borislav Hristov

Dimyadi, Johannes Alwi Wibisono

Dinamarca, Carlos

Dincă, Marius Sorin

Dincer, M. Sezai

Dindorf, Ryszard

Ding, Kai

Ding, Xinrui

Ding, Zhao

Dinh, Toan

Dini, Pierpaolo

Dinis Gaspar, Pedro

Dinis, Maria Alzira Pimenta

Dinita, Alin

Dinulovic, Dragan

Dion, Lukas

Dionisi, Davide

Diouf, Boucar

Dipasquale, Chiara

Dipu, Arnoldus Lambertus

Dishari, Shudipto Konika

Ditl, Pavel

Diwekar, Urmila M.

Dixon, David A.

Dixon, James

Dizqah, Arash M.

Djagarov, Nikolay

Djerdir, Abdeslem

Djeziri, Mohand

Djordjevic, Boban 
Djourelov, Nikolay

Djurović, Siniša

Długosz, Adam

Dmitrievskii, Vladimir

Dmitruk, Anna

Do Paço, Ana

Do, Jing-Shan

Do, Thang Nam

Do, Wanglok

Doan, Dat

Dobák, Samuel

Dobre, Alexandru

Dobre, Ciprian

Dobrescu, Lidia

Dobrev, Ivan

Dobreva, Antoaneta

Dobrocky, David

Dobroka, Mihaly

Dobrucky, Branislav

Dobrzanska, Magdalena

Dobrzycki, Arkadiusz

Dobzhanskyi, Oleksandr

Dogančić, Dragana

Dogaru, Vasile

Dolacek-Alduk, Zlata

Dolega, Boguslaw

Dołęga, Waldemar

Dolfing, Jan

Dolinska, Silvia

Dolnik, Bystrik

Dolníková, Erika

Dolz, Vicente

Dołżyńska, Magdalena

Domagała, Sławomir

Domanska, Joanna

Domański, Adam

Dombek, Grzegorz

Domicián, Máté

Domine, Marcelo E.

Domingo-Irigoyen, Silvia

Domingues, Nuno

Dominguez, Carlos Ortiz

Dominguez, Ruth

Dominguez, Sergio

Domínguez-Amarillo, Samuel

Domínguez-García, José Luis

Domínguez-Navarro, Jose Antonio

Dominguez-Robles, Juan

Domjan, Suzana

Domokos, Gyore

Donaldson, A. Burl
Donat, Felix

Donateo, Teresa

Dong, Feng

Dong, Jianning

Dong, Longjun

Dong, Ming

Dong, Mingdong

Dong, Qiuchen

Dong, Yiwei

Dong, Zhizhong

Dongare, Pratiksha

Donnal, John

Donskoy, Igor

Dooner, Mark

Doost Mohammadian, Hamid

Dopazo, Gonzalo Astray

Dorado, M. Pilar

Dorao, Carlos A.

Doretti, Luca

Dòria-Cerezo, Arnau

Dorneles Callegaro, Alan

Doroudchi, Elahe

Dos Santos, Pedro Mendonça

Dose, Wesley

Dosoftei, Constantin Catalin

Dosta, Joan

Dostal, Leo

Doucek, Petr

Douglas, Christopher

Doujak, Eduard

Doulos, Lambros T.

Doumiati, Moustapha

Dounis, Anastasios

Doustdar, Omid

Douthwaite, Mark

Douziech, Melanie

Dovì, Vincenzo

Dovjak, Mateja

Drabczyk, Anna

Drachal, Krzysztof

Drąg, Paweł

Dragan, Dejan

Drago, Dolinar

Dragomir, Alin

Dragomir-Stanciu, Daniel

Dragos, Machidon

Drava, Giuliana

Draxl, Caroline

Drezewski, Rafal

Drgoňa, Ján

Drgona, Peter 
Driha, Oana Madalina

Drikakis, Dimitris

Drisya Alex, Thumba

Droutsa, Kalliopi

Drozd, Jan

Drozd, Zdenek

Drozdz, Stanislaw

Druetta, Pablo

Druica, Elena

Drulis, Henryk

Drury, Dave

Drusa, Marián

Drygała, Aleksandra

Drygała, Izabela Joanna

Drzeżdżon, Joanna

Drzymała, Tomasz

Dsoke, Sonia

$\mathrm{Du}$, Boxue

$\mathrm{Du}, \mathrm{Hu}$

$\mathrm{Du}$, Jiangtao

Du, Sijun

Du, Yang

Du, Ya-Ping

Du, Yuncheng

Du, Zhiyan

Duan, Dongliang

Duan, Jie

Duan, Lunbo

Duan, Nan

Duan, Shengyu

Duarte Correia, Sérgio

Duarte Marafona, José

Dube, Patrick

Dubé, Yves

Dubey, Deepak Kumar

Dubey, Harishchandra

Dubuis, Guy

Duchaud, Jean-Laurent

Duchesne, Marc

Duchon, Markus

Duda, Kamil

Duda, Piotr

Dudás, László

Dudek, Grzegorz

Dudek, Magdalena

Dudhia, Jimy

Dudojc, Boleslaw

Dudyński, Marek

Duer, Stanisław

Dufo-López, Rodolfo

Dufour, Anthony
Duić, Neven

Duine, Gert-Jan

Dulău, Lucian Ioan

Dulebenets, Maxim A.

Dulf, Eva H.

Dumbrava, Stefan

Dumbrava, Virgil

Dumciuviene, Daiva

Dumic, Emil

Dumitrache, Alexandru

Dumitran, Laurenţiu Marius

Dumitras, Diana

Dumitrascu, Monica

Dumitrescu, Bogdan Andrei

Dumitrescu, Mariana

Dumitriu, Mădălina

Dumitru, Cristian-Dragoş

Dunderdale, Gary J.

Dundulis, Gintautas

Dunford, William G.

Dung, Le The

Dung, Nguyen Dinh

Dunn, Robert O.

Duong, Minh Quan

Duplančić Leder, Tea

Duprat, Véronique

Dupuis, Pascal

Duque-Perez, Oscar

Duraes, Joao

Durán Aranda, Eladio

Durana, Pavol

Durčanský, Peter

Durczak, Karol

Đurđević, Dinko

Durdyev, Serdar

Đurin, Bojan

Ďuriš, Lukáš

Ďuriš, Stanislav

Durmus, Dorukalp

Duro Carralero, Natividad

Durovsky, Frantisek

Durrani, Faisal

Dushaishi, Mohammed F. Al

Dusseault, Maurice

Dutt, Nilanjana

Dutta, Animesh

Dutta, Biswanath

Dutta, Gaurab

Dutta, Rabijit

Dutta, Rukmi

Dutta, Saikat 
Dutta, Sandip

Duval, Michel

Dvorkin, Jack

Dwamena, Amos

Dwivedi, Ashutosh Dhar

Dyatkin, Boris

Dybkowski, Mateusz

Dyjakon, Arkadiusz

Dyka, Ireneusz

Dylewski, Robert

Dzhonova, Daniela

Dzida, Marzena

Dziechciarz, Józef

Dziedzic, Andrzej

Dziedzic, Krzysztof

Dziekońska-Kubczak, Urszula

Dzierwa, Piotr

Dzikuć, Maciej

Dzikuć, Maria

Dziok, Tadeusz

Džiugys, Algis

Dziurdzia, Piotr

Dziurzyński, Wacław

Eames, Matt E.

Eberle, Ulrich

Ebong, Abasifreke U.

Ebrahimi Salari, Mahdi

Ebrahimi, Amin

Ebrahimi, Kambiz

Ebrahimi, Mehdi

Ebrahimiaqda, Elham

Echarri-Iribarren, Victor

Eckert, Andrew

Eden, Tim

Edlmann, Katriona

Edoh, Ayaboe

Edomwonyi-Otu, Lawrence C.

Edu, Tudor

Eduardo Espitia, Helbert

Eensaar, Agu

Effenberger, Mathias

Eftekhari, Behzad

Efthimiou, George

Efthymiou, Loizos

Efthymiou, Venizelos

Egea Alvarez, Agusti

Egedy, Attila

Egerer, Jonas

Egi, Yunus

Eguchi, Kei

Egwebe, Augustine
Ehnberg, Jimmy

Ehrenberg, Helmut

Ehrmann, Andrea

Ehsan, Ali

Ehsan, Mostafavi

Eichberger, Bernd

Eisa, Sameh A.

Eitelberg, Georg

Ejsmont, Krzysztof

Ekanayake, Janaka

Ekkad, Srinath

Ekonomou, Lambros

Ekpeni, Leonard

Ekpo, Sunday

El Aroudi, Abdelali

El Hariri, Mohamad

El Mariachet, Jorge

El Sachat, Alexandros

Elahi, Hassan

Elakneswaran, Yogarajah

Elasha, Faris

El-Bayeh, Claude

Elbouchikhi, Elhoussin

Elduque, Daniel

Elena, Lucchi

Elena, Tiuc Ancuţa

Elforjani, Mohamed

El-Hadedy, Mohamed

Elhadidi, Basman

Elia, Stefano

Elias, Said

Eliche Quesada, Dolores

Elkamel, Ali

Elkatatny, Salaheldin

El-kharbachi, Abdel

El-Kharouf, Ahmad

Elle, Morten

Ellis, Eugenia Victoria

Elmasides, Costas

Elmi, Chiara

Elmogy, Ahmed

Elmore, R. Douglas

Elmoselhy, Salah

Elnajjar, Emad

Elreedy, Ahmed

Elsaiah, Salem

El-Shahat, Adel

Elsner, Witold

Elsts, Atis

Elwell, Cliff

EL-Zahar, Essam R. 
Elzohairy, Ayman

Emad, Muhammad Zaka

Emadi, Arezoo

Emblen-Perry, Kay

Emery, Isaac

Emes, Matthew

Emilia, Paone

Emmanuel, Tatakis

Emodi, Nnaemeka

Enalou, Hossein Balaghi

Encinar, José María

Endreny, Theodore

Enescu, Diana

Engelberger, Antonia

Engelen, Peter Jan

English, Niall

Enoki, Koji

Enríquez Pérez, Esther

Enyashin, Andrey

Eramo, Vincenzo

Eras-Almeida, Andrea A.

Erdem, Emre

Erden, Fatih

Erdogan, Nuh

Eregno, Fasil Ejigu

Eremin, Mikhail O.

Erhart, Tobias

Ermoshkin, Alexey V.

Erokhin, Vasilii

Errandonea, Daniel

Errico, Vito

Ersland, Geir

Ersoy, Aksel

Escalante, Jaime

Escalera, Alberto

Escorihuela, Jorge

Eskandari Torbaghan, Mehran

Eskandari, Mohsen

Eskander, Shaikh

Esmaeely Neisiany, Rasoul

Esmaeili, Sajjad

Espinosa Fernandez, Almudena

Espinosa, Antonio Garcia

Espinosa, Eduardo

Espinosa, Manuel Jesús

Esposito, Christian

Esquivias, Paula

Estanqueiro, Ana

Esteban, Maria Dolores

Estel, Lionel

Estevadeordal, Jordi
Esteves, Bruno M.

Estokova, Adriana

Estrada-López, Johan J.

Eszter, Hubainé Tóth

Esztergár-Kiss, Domokos

Etacheri, Vinodkumar

Etz, Radu

Euiseok, Hwang

Euler-Rolle, Nikolaus

Euverink, Gert-Jan

Evangelista, Pietro

Evangelisti, Luca

Evans, Nathaniel

Evdokinov, Oleg

Eveloy, Valerie

Evola, Gianpiero

Evsutin, Oleg

Ewert, Pawel

Exizidis, Lazaros

Exposito, Antonio Jose

Ezazi, Mohammadamin

Eze, Vincent

Ezzati, Sattar

Fabbri, Kristian

Fabio, Mandrile

Fabrizio, Enrico

Fábry, Stanislav

Facchini, Francesco

Fachrizal, Reza

Faddel, Samy

Fagarasan, Ioana

Faggianelli, Ghjuvan Antone

Fahim, Muhammad

Fahs, Marwan

Faia, Ricardo

Failla, Giuseppe

Failler, Pierre

Faiz, Mohinudeen

Fajri, Poria

Fala, Nicoletta

Falabretti, Davide

Falahi, Ghazal

Falcão, D. S.

Falcucci, Giacomo

Faleiro, Eduardo

Falini, Antonella

Falsafioon, Mehdi

Falsone, Giovanni

Famoso, Fabio

Fan, Huan-Jung

Fan, Jiajie 
Fan, Liyang

Fan, Miao

Fan, Xiaoyuan

Fan, Xin

Fan, Yee-Van

Fan, Yilin

Fancher, Chris

Fanciulli, Carlo

Fandi, Ghaeth

Fandino, Olivia

Fang, Chen

Fang, Ruixian

Fangrat, Jadwiga

Fanizza, Elisabetta

Fanizzi, Francesco Paolo

Fantozzi, Fabio

Fara, Laurentiu

Faraci, Carla

Farag, Hany

Farago, Paul

Farah, Paolo Davide

Farahani, Abolfazl Sousanabadi

Faranda, Roberto

Farantatos, Evangelos

Farcas, Anca Corina

Farcasanu, Ileana C.

Farhangi, Mohsen

Faria, Pedro

Farina, Ilenia

Farinha, Fatima

Farinha, José Torres

Farjana, Shahjadi Hisan

Farkaš, Branimir

Farkas, Csaba

Farkoush, Saeid Gholami

Farlik, Jan

Farmann, Alexander

Farough, Aida

Farouq Ali, S. M.

Farraji, Hossein

Farrier Alexander, Luke

Farris, Thomas N.

Farrokhifar, Meisam

Farroni, Flavio

Faruque, Md Omar

Farzan Moghaddam, Ali

Farzaneh, Hooman

Fasano, Matteo

Fascista, Alessio

Fassas, Athanasios P.

Fatás, Enrique
Fathi, Soheil

Fatigati, Fabio

Fatih Tuğan, Murat

Fattah, Islam Md Rizwanul

Favero, Alice

Fayaz, Mohammadreza

Fazeli, Hossein

Fazeli, Reza

Fecarotta, Oreste

Feczkó, Tivadar

Fedele, Lorenzo

Fediuk, Roman

Fedorko, Gabriel

Fedorov, Ruslan V.

Feier, Ioan

Feigl, Gábor

Feijóo Lorenzo, Andrés Elías

Feijóo, Andrés

Fekete, Krešimir

Fekete, Mária Magdolna

Fekih, Afef

Feldhoff, Armin

Feldhoff, Thomas

Felez, Jesus

Felgueiras, Carlos

Felis Enguix, Ivan

Feliziani, Mauro

Felseghi, Raluca Andreea

Femenias, Paula

Feng, Cong

Feng, Guojin

Feng, Huicheng

Feng, Ju

Feng, Qicheng

Feng, Ruimin

Feng, Wentao

Feng, Xiang

Feng, Yanbiao

Feng, Yin

Feng, Zhiyuan

Fennell, Pamela

Fentaye, Amare Desalegn

Feo, Alessandra

Fera, Marcello

Ferdelji, Nenad

Ferdes, Mariana

Ferdous, Wahid

Fereidountabar, Amirhossein

Fernandes, Célio

Fernandes, Marco S.

Fernandes, Paulo J. T. 
Fernández Gámez, Manuel Angel

Fernández García, Francisco Javier

Fernandez Martinez, Roberto

Fernandez Oro, Jesus Manuel

Fernandez, Carlos

Fernandez, Efren

Fernández, Francisco José Vivas

Fernández, Rafael Sebastián

Fernández, Roberto Álvarez

Fernandez, Rosa Maria

Fernández-Agüera, Jessica

Fernandez-Balbuena, Antonio Alvarez

Fernandez-Basso, Carlos

Férnandez-Batanero, Jose M.

Fernández-Campusano, Christian

Fernández-Cardador, Antonio

Fernández-Cerero, Damián

Fernandez-Gamiz, Unai

Fernandez-Garcia, Javier

Fernandez-Garcia, Raul

Fernández-Isabel, Alberto

Fernández-Jiménez, Luis Alfredo

Fernández-Lázaro, Fernando

Fernandez-Marchante, Carmen M.

Fernández-Ramírez, Luis M.

Fernandez-Vidal, Severo Raul

Fernández-Vilas, Ana

Fernández-Yáñez, Pablo

Fernando, Gerard

Féron, Damien

Feron, Krishna

Ferradosa, Tiago

Ferrández, Daniel

Ferrando, Riccardo

Ferrantelli, Andrea

Ferranti, Francesco

Ferrara, Giuseppe

Ferrara, Massimiliano

Ferrari, Federica

Ferrari, Lorenzo

Ferrarini, Giovanni

Ferraris, Giovanni

Ferreira Lousado, José Paulo

Ferreira Morais, Lenin Martins

Ferreira, Alexandre

Ferreira, Angela

Ferreira, António

Ferreira, Fernando J. T. E.

Ferreira, Filipa Maria Santos

Ferreira, Francisco

Ferreira, Joao
Ferreira, Joaquim

Ferreira, Jorge

Ferreira, Luís Andrade

Ferreira, Paulo

Ferrero, Andrea

Ferrero, Domenico

Ferretti, Ivan

Ferrieux, Jean-Paul

Ferro, Giulio

Ferruzzi, Gabriella

Few, Sheridan

Fezi, Kyle

Fialová, Simona

Fiaschi, Daniele

Ficarella, Antonio

Ficco, Giorgio

Fichera, Alberto

Ficker, Tomas

Fidani, Cristiano

Fidelibus, Corrado

Fidlerova, Helena

Fifariz, Reynaldy

Fijałkowski, Karol

Fijorek, Kamil

Fike, Matej

Fiket, Zeljka

Filgueira-Vizoso, Almudena

Filip, Ioan

Filipiak, Michał

Filipiak, Sara

Filipović, Vladimir

Filipowicz, Mariusz

Filippov, Oleg A.

Fillion, Marie-Hélène

Filo, Grzegorz

Filonenko, Konstantin

Finck, Steffen

Findorák, Róbert

Fine, Jamie

Finegan, Donal P.

Finesso, Roberto

Finn, Donal

Finzi, Alberto

Fioravanti, Ambra

Fiore, Pierfrancesco

Fiore, Salvatore

Fiore, Silvia

Fiori, Chiara

Fiori, Luca

Fiorillo, Fausto

Fioriti, Davide 
Fiorito, Francesco

Firețeanu, Virgiliu S.

Firlag, Szymon

Firoozabadi, Abbas

Fisch, Michael

Fischer, Hartmut R.

Fischer, Helen

Fischer, Stefan

Fischer, Szabolcs

Fischer, Thomas

Fischlschweiger, Michael

Fiser, Rastko

Fitamo, Temesgen

Fitzgerald, Breiffni

Fitzpatrick, Colin

Fixen, Kathryn

Flamini, Fulvio

Flanagan, Ted

Flandin, Lionel

Flegner, Patrik

Fleming, Paul

Fletcher, Tom

Fleur, Lina La

Flizikowski, Jozef

Floors, Rogier

Floquet, Pascal

Florencias Oliveros, Olivia

Flores, Robert J.

Flores-Arias, Jose-Maria
Fonod, Robert

Fonseca I Casas, Pau

Fonseca, José

Fonseca, Luis

Font Palma, Carolina

Fontana, Daniela

Fontanesi, Claudio

Fontani, Daniela

Foraboschi, Paolo

Forniés, Eduardo

Foroozandeh, Zahra

Fort, Ada

Fořt, Jan

Fortier, Marie-Odile

Fortmann, Charles

Fortuna, Luigi

Fossa, Marco

Foteinis, Spyros

Foti, Daniel

Foti, Salvatore

Fotouhi, Abbas

Foucher, Fabrice

Fouilloy, Alexis

Founti, Maria

Fourneau, Jean-Michel

Fox, Richard

Főző, Ladislav

Fracastoro, Giovanni Vincenzo

Fraczyk, Justyna

Florescu, Adriana (Politehnica University of

Bucharest, Romania)

Florescu, Adriana (Transilvania University

of Brasov, Romania)

Florez, Horacio

Floricau, Dan

Florimond, Guéniat

Florio, Ciro

Florita, Anthony

Florkowski, Marek

Floros, George

Flotats, Xavier

Flox, Cristina

Fnadi, Mohamed

Fragkos, Panagiotis

Fragliasso, Francesca

Fragos, Vassilios

Fraile-Ardanuy, Jesus

Franchina, Nicoletta

Francik, Sławomir

Franco, Alessandro

Franco, Christian

Franco, John F.

Franco, Vicente

François, Baptiste

Francois, Bruno

François, Gregory

Frank, Andrew A.

Franković, Dubravko

Frankowski, Jakub

Franquet, Erwin

Frantz, Jesse

Franzitta, Vincenzo

Frasca, Francesca 
Frate, Claudio Albuquerque

Frattini, Domenico

Frattolillo, Andrea

Fratu, Octavian

Frazier, Robert Scott

Frazzica, Andrea

Frederick, Robert A.

Fredianelli, Luca

Frei, Stephan

Freidoonimehr, Navid

Freire-Lista, David

Freitas Dutra, Lorena

Freitas, Manuel João Sepúlveda Mesquita

Freixa Terradas, Jordi

Fresner, Johannes

Frias, Pablo

Friedrichs, Jens

Friess, Wilhelm Alexander

Frigaard, Peter

Frigura-Iliasa, Flaviu Mihai

Friis, Henrik

Friman, Hen

Frincu, Marc

Fritzkowski, Paweł

Frivaldsky, Michal

Frizziero, Leonardo

Frizzo Stefenon, Stéfano

Frodeson, Stefan

Froio, Antonio

Frosini, Lucia

Fruggiero, Fabio

Früh, Wolf-Gerrit

Fryc, Irena

$\mathrm{Fu}, \mathrm{Bo}$

$\mathrm{Fu}$, Chao

$\mathrm{Fu}$, Guopeng

$\mathrm{Fu}$, Hailing

$\mathrm{Fu}$, Jingying

$\mathrm{Fu}, \mathrm{Li}$

$\mathrm{Fu}$, Wei

$\mathrm{Fu}$, Weinong

$\mathrm{Fu}$, Xingang

$\mathrm{Fu}$, Xiuwen

$\mathrm{Fu}$, Yangyang

$\mathrm{Fu}$, Yingkun

$\mathrm{Fu}$, Zhuojia

Fuchs, Gerhard

Fuente, María J.

Fuentes-Bargues, José Luis

Fujii, Yoshiaki

Fujimoto, Daisuke
Fujimoto, Hiroyuki

Fujimoto, $\mathrm{Yu}$

Fujita, Goro

Fulignati, Sara

Fumagalli, Alessio

Funari, Marco Francesco

Furgal, Jakub

Furió Ortega, María Dolores

Furlani Bastos, Alvaro

Furse, Samuel

Fürst, Jiří

Furusato, Tomohiro

Fusco, Giuseppe

Futa, Barbara

Fydrych, Dariusz

Gabaldón, Antonio

Gabaldon-Estevan, Daniel

Gabash, Aouss

Gabbar, Hossam A.

Gabdullin, Nikita

Gabioud, Dominique

Gabitto, Jorge

Gabl, Roman

Gábor, Szepesi L.

Gabriel, Velu

Gabriela, Dobrotă

Gabrielli, Fabrizio

Gad-Briggs, Arnold

Gadedjisso-Tossou, Agossou

Gadipelli, Srinivas

Gadola, Marco

Gaetani, Isabella

Gafurov, Marat

Gagaoudakis, E.

Gagliano, Antonio

Gaiceanu, Marian

Gajewski, Andrzej

Gajic, Dragoljub

Gałaj, Jerzy

Galamboš, Michal

Galan, Ana-Maria

Galán, Andrés Ramos

Galanis, Athanasios

Galati, Antonino

Gałczyńska, Małgorzata

Galiano, Antonio Berná

Galić, Ivo

Galić, Mario

Galindo, Gloria Gea

Galindo-Torres, Sergio A.

Galinsky, Nathan 
Galipeau, David W.

Galkin, Ilya A.

Gałko, Grzegorz

Galkowski, Krzysztof

Galla, Stanislaw

Gallerano, Francesco

Gallo, Daniele

Gallo, Mauro

Galluzzi, Renato

Galos, Joel

Galos, Krzysztof

Galvan Diez, Eduardo

Galvão, João Rafael

Galvin, Raymond

Galvita, Vladimir V.

Gamaleev, Vladislav

Gambaruto, Alberto

Gambelli, Alberto Maria

Gamboa, Paulo José Duarte Landeiro

Gamez Montero, Pedro Javier

Gamidullaeva, Leyla

Gamiño-Arroyo, Zeferino

Gamulin, Ozren

Gancarz, Marek

Gandiaga, Iñigo

Gandoman, Foad

Gandzha, Segey

Ganea, Daniel

Ganesan, A.

Ganesh, R. Sankar

Gani, Terry

Ganjkhanlou, Yadolah

Gao, Fei

Gao, Feng

Gao, Jianbing

Gao, Linda

Gao, Ningshengjie

Gao, Shangce

Gao, Wei

Gao, Weijun

Gao, Yabiao

Gao, Yue

Gao, Zhiwei

Garapati, Nagasree

Garau, Michele

Garbayo, Inés

Garbowski, Tomasz

Garbuio, Lauric

Garcia Cascales, Maria Socorro

García Fernández, José Luis

García Márquez, Fausto Pedro
García Martín, Juan Francisco

García Rodríguez, Juan

García Sánchez, Manuel

García, Ana Salomé

Garcia, Antoni

García, Carmelo

Garcia, Edenise

Garcia, Gonzalo

Garcia, João

García, Manuel Pérez

García, Miguel

García, Miguel Torres

García, Roberto

Garcia, Sebastián

Garcia-Almiñana, Daniel

Garcia-Amorós, Jordi

García-Armingol, Tatiana

Garcia-Bravo, José M.

García-Cerrada, Aurelio

García-Contreras, Reyes

García-Delgado, Carlos

García-Díaz, Juan Carlos

García-Fernández, Berta

García-Fogeda, Pablo

García-Fuentes, Miguel Á.

García-García, Agustín

García-Gracia, Miguel

García-Gusano, Diego

García-Herrero, Susana

García-López, Francisco De Paula

García-Magariño, Iván

García-Ortega, Beatriz

Garcia-Perez, Arturo

García-Torres, Félix

Gardijan Kedžo, Margareta

Gardner, Matthew

Gardy, Jabbar

Garibaldi, Luigi

Garicano-Mena, Jesús

Garinei, Alberto

Garma, Tonko

Garmendia, Iñaki

Garrido Jurado, Juan

Garrido Picazo, María Piedad

Garrido, Gustavo Nofuentes

Garrido, Lara Febrero

Garriga, Rosa

Garro, Unai

Garroni, Sebastiano

Garrote, Gil

Garsztka, Przemysław 
Gaska, Krzysztof

Gaspar, Cristina

Gaspar, Peter

Gaspari, Jacopo

Gašparović, Mateo

Gatt, Peter

Gatteschi, Valentina

Gatti, Manuele

Gatti, Teresa

Gattia, Daniele Mirabile

Gatto, Andrea

Gatto, Gianluca

Gaudioso, Elena

Gaurina-Međimurec, Nediljka

Gautam, Sneha

Gava, Oriana

Gavaises, Manolis

Gavanas, Nikolaos

Gavioso, Roberto M.

Gavrilas, Mihai

Gavrilova, Natalia

Gavrilova, Tatiana A.

Gawin, Dariusz

Gawlik, Lidia

Gawlińska, Katarzyna

Gawor, Łukasz

Gawrylczyk, Konstanty Marek

Gayner, Chhatrasal

Gazdzicki, Pawel

Ge, Baoming

Ge, Cui

Ge, Jun Cong

$\mathrm{Ge}$, Xinlei

Ge, Yunfeng

Gebhardt, Paul

Gebremariam, Shemelis Nigatu

Gebremedhin, Alemayehu

Géczy, Attila

Gedikli, Ersegun Deniz

Gediminas, Vaičiūnas

Geethanjali, M.

Geisbüsch, Jörn

Geise, Andrzej

Gejdoš, Miloš

Gejji, Rohan

Gelashvili, Vera

Gelegenis, John

Gelman, Len

Gemar, Mason

Genco, Filippo

Gendek, Arkadiusz
Generowicz, Agnieszka

Geng, Guangchao

Geng, Jianzhao

Genovese, Francesco

Genovese, Giuseppa

Genovese, Maria Erminia

Gentile, Sabrina

Gentili, Francesco

Georgakellos, Dimitrios

Georgantzinos, Stelios K.

George, Ioannidis

George, Thomas F.

Georgescu, Andrei-Mugur

Georgeta, Soava

Georgiadis, Teodoro

Georgiev, Daniel G.

Georgilakis, Pavlos S.

Georgiou, Emmanuel

Georgious, Ramy

Georgoudas, Ioakeim

Gerbens-Leenes, Winnie

Gerber, Philippe

Gerboni, Raffaella

Gercek, Cihan

Gerding, Enrico

Geri, Alberto

Gericota, Manuel

German, Konstantin E.

German-Galkin, Sergey

Germanos, Vasileios

Gerogiorgis, Dimitrios

Geszke-Moritz, Małgorzata

Geth, Frederik

Gevorkov, Levon

Geza, Husi

Ghaani, Mohammad Reza

Ghafouri, Mohsen

Ghaisas, Niranjan

Ghamari, Mohsen

Ghanbarian, Behzad

Ghanes, Malek

Ghani, Arfan

Ghannoum, AbdulRahman

Ghedira, Sami

Gheitaghy, Amir Mirza

Gheju, Marius

Gheorghe, Grigoras

Gheorghiu, Andrei

Gherardi, Francesca

Ghezehei, Solomon

Ghiani, Emilio 
Ghiara, Giorgia

Ghods, Masoud

Ghofrani, Ali

Gholamalizadeh, Ehsan

Gholami Farkoush, Saeid

Gholikhani, Mohammadreza

Gholizad, Babak

Gholz, Eugene

Ghorbani, Morteza

Ghoreishi, Seyede Fatemeh

Ghoreyshi, Seyed Mohammad

Ghosh, Aritra

Ghosh, Prasanta K.

Ghosh, Ruchira

Ghuiaus, Christian

Giacomin, João Carlos

Giacomo Prina, Matteo

Giancola, Emanuela

Gianfranco, Fenu

Gianluca, Palermo

Giannakis, Konstantinos

Giannakopoulos, Dionysios

Giannella, Venanzio

Giannetti, Niccolò

Gianni, Bidini

Giansoldati, Marco

Giaourakis, Dimitrios G.

Giardina, Mariarosa

Giasin, Khaled

Giberti, Hermes

Gibiino, Gian Piero

Gichuhi, Wilson

Gidaspow, Dimitri

Gidon, Dogan

Giebel, Gregor

Giebeler, Lars

Gierszewski, Mateusz

Gies, Valentin

Giglio, Carlo

Giglio, Emanuele

Giglioli, Romano

GIl, Bartosz

Gil, David

Gil, Nuno

Gilbert, James M.

Gil-de-Castro, Aurora

Gil-Garcia, Isabel Cristina

Gilioli, Andrea

Gill, Sukhpal Singh

Gillard, Philippe

Gilles, Notton
Gil-Lopez, Sergio

Gillot, Frédéric

Gilmore, Colin

Gilsu, Choi

Giltinan, Joshua

Gimenez, Antonio

Giménez-Gómez, Pablo

Ginart, Antonio

Giordano, Nicolo

Giorgi, Giuseppe

Giorgio, Guariso

Giorgio, Ivan

Giorno, Lidietta

Giostri, Andrea

Giosuè, Chiara

Giouri, Katerina

Giovannelli, Ambra

Girnik, Ilya

Girolami, Laurence

Girolami, Marco

Girovský, Peter

Giubileo, Filippo

Giuffre, Ottavia

Giuffrida, Nadia

Giuffrida, Salvatore

Giuliano, Aristide

Giungato, Pasquale

Giuseppe, Gibertini

Giuseppi, Alessandro

Giustiniani, Michela

Givaki, Kamyab

Gkavanoudis, Spyros

Gkoumas, Konstantinos

Gkritza, Konstantina

Gladysz, Pawel

Gładyszewska-Fiedoruk, Katarzyna

Glaser, Rainer

Glassley, William Edward

Glavas, Hrvoje

Glavic, Mevludin

Glazar, Vladimir

Glebov, Evgeni M.

Gleich, Dusan

Glema, Adam

Glensk, Barbara

Glicksman, Allen

Glicksman, Leon

Gliedt, Travis

Gligor, Adrian

Gloaguen, Frederic

Glos, Jan 
Glover, Paul

Głowacki, Tadeusz

Glowacz, Adam

Glushkov, Dmitrii O.

Glynn, Peter

Gnaciński, Piotr

Gnoni, Maria Grazia

Go, Yun Ii

Gobert, Julie

Gockenbach, Ernst

Godbout, Stéphane

Godina, Radu

Godinez, Fernando

Godlewska, Joanna

Godoy, Antonio José Calderón

Godyń, Katarzyna

Gogolewska, Anna

Gogonea, Rodica-Manuela

Gogos, Christos

Göhlich, Dietmar

Goit, Jay Prakash

Goktas, Mustafa

Golak, Sławomir

Gołaszewski, Janusz

Goldmann, Daniel

Gołębiewski, Jarosław

Gołębiowska, Justyna

Golebiowski, Leslaw

Gołębiowski, Piotr

Golewski, Grzegorz Ludwik

Golgovici, Florentina

Goli, Preetham

Golijanek-Jędrzejczyk, Anna

Golimowski, Wojciech

Golmohamadi, Hessam

Golob, Marjan

Golovanov, Dmitry

Golub, Igor

Golub, Victor V.

Golubev, Oleg V.

Golubovskii, Yuri

Golzar, Farzin

Gombarska, Daniela

Gomes, Ana P.

Gomes, Daniel

Gomes, João Castro

Gomes, Luis

Gomes, Phillipe Vilaça

Gómez Barrios, Xiomar

Gomez Camacho, Carlos Enrique

Gómez Fernández, Juan F.
Gomez Melgar, Sergio

Gómez Vela, Francisco A.

Gómez Vilchez, Jonatan J.

Gómez, Manuel Romero

Gomez, Ricardo S.

Gómez, Víctor Alonso

Gómez, Xabier

Gómez-Calvet, Roberto

Gómez-Llanos, Eva

Gómez-Martín, M. Esther

Gómez-Ortiz, David

Gomez-Plana, Antonio

Gomez-Soriano, Josep

Gömöry, Fedor

Goncalves, Alexandre

Gonçalves, Ana L.

Gonçalves, Artur

Goncalves, Olivier

Gonçalves, Renato

Gondolini, Angela

Gonet, Andrzej

Gong, Jian

Gong, Jiawei

Gong, Jue

Gonos, Ioannis F.

González De La Rosa, Juan-José

Gonzalez De Los Reyes, Rafael Corsino

González García, Mariano

González Horcas, Sergio

Gonzalez Junca, Arnau

González Marcos, Ana

González Pérez, Isaías

González Pino, Iker

González Prieto, José Francisco

González, Ana Tejero

González, Aránzazu Galán

González, Asier

González, Fermín Barrero

Gonzalez, Fernando

González, Hernán

González, Javier Serrano

González, Jose

Gonzalez, Juan Diaz

González, Juan Félix

Gonzalez, Maria Manuela Prieto

González-Briones, Alfonso

González-Díaz, Benjamín

González-Díaz, Diego

González-Domínguez, Pablo Ignacio

González-Gómez, Manuel

Gonzalez-Lezcano, Roberto Alonso 
Gonzalez-Longatt, Francisco

González-Morán, Cristina

Gonzalez-Pedro, Victoria

González-Peña, David

González-Prieto, Ángel

González-Prieto, Ignacio

González-Vila, Álvaro

Goodman, Angela

Goodship, Vannessa

Gopalan, Harish

Gopalan, Kaliappan

Goraj, Weronika

Gorawska, Anna

Gorbenkova, Elena

Gorbunova, Ella M.

Gordillo, Francisco

Gordon, Jeffrey

Górecki, Krzysztof

Górecki, Paweł

Gori, Elena

Gori, Paola

Gorji, Nima E.

Gorji, Saman Asghari

Gormally, Alexandra

Górniak, Aleksander

Górnicki, Krzysztof

Górski, Jarosław

Gortschakow, Sergey

Goryl, Wojciech

Gosar, Žiga

Gośliński, Tomasz

Gosselin, Louis

Goswami, Arghya

Goswami, Rajib

Göteman, Malin

Goteti, Naga Srujana

Goto, Mika

Goto, Satoru

Gotor-Fernández, Vicente

Gotovtsev, Pavel

Gotti, Claudio

Gottschalk, Axel

Goubej, Martin

Goudarzi, Navid

Gough, Hannah

Goula, Maria A.

Goulas, Konstantinos

Gould, Benjamin D.

Goulet, Marc-Antoni

Goushcha, Oleg

Gouveia, Eduardo M.
Gouveia, Maria Do Castelo

Govaerts, Jonathan

Gozalbo Rovira, Roberto Vicente

Gozdowiak, Adam

Graba, Mariusz

Grabara, Janusz

Grabarczyk, Robert

Graber, Giuseppe

Graber, Lukas

Grabowski, Paweł

Grabowski, Wojciech

Grab-Rogaliński, Karol

Gradeci, Klodian

Gradov, Oleg M.

Gradova, Margaret

Gradziuk, Piotr

Graf, Frank

Gragnani, Gian Luigi

Grajek, Henryk

Grajewski, Jakub

Grammelis, Panagiotis

Grams, Jacek

Grana, Anna

Graña-López, Manuel Ángel

Grasby, Stephen E.

Grasso, Emanuele

Gravalidis, Christoforos

Gravdahl, Jan Tommy

Grazieschi, Gianluca

Grażyna, Żukowska

Grdenić, Goran

Greco, Adriana

Greco, Marco

Grecu, Eugenia

Greenman, John

Greenough, Rick

Grega, Robert

Gregov, Goran

Greppi, Matteo

Grewal, Kuljeet Singh

Grgic, Davor

Grieco, Giuseppe

Grieves, Michael

Griffiths, Luke

Griffiths, Philip

Griggs, Thomas

Grigolato, Stefano

Grigore, Roxana

Grigorev, M. A.

Grigorova-Shtarbeva, Tsvetana

Grillo, Federico 
Grillo, Samuele

Grimaccia, Francesco

Grimm, Alejandro

Grindeanu, Iulian

Grishagin, Vladimir A.

Grisolia, Giulia

Gritsenko, Dmitry

Griva, Giovanni Battista

Grochowicz, Marta

Gródek-Szostak, Zofia

Gróf, Gyula

Gromada, Magdalena

Gromek, Pawel

Gronchi, Paolo

Groniewsky, Axel

Groot, Jens

Gros, Ioana-Cornelia

Grossi, Luigi

Grossmann, Ignacio

Groza, Robert

Grube, Thomas

Grudzińska, Magdalena

Gruenwald, Johannes

Grün, Thorsten

Gruszka, Konrad

Gryaznevich, Mikhail P.

Grygierek, Joanna

Gryning, Sven-Erik

Grzebielec, Andrzej

Grzeczka, Grzegorz

Grzejszczak, Piotr

Grzybowski, Hubert

$\mathrm{Gu}$, Chunyang

$\mathrm{Gu}$, Fengshou

$\mathrm{Gu}$, Jianyu

$\mathrm{Gu}$, Wenting

Gualous, Hamid

Gualtieri, Giovanni

Guariso, Giorgio

Guarnieri, Massimo

Gubina, Andrej

Guchhait, Rekha

Guérard, Guillaume

Guerin, Katia

Guerreiro, Maria João Simas

Guerrero-Rodríguez, Néstor Francisco

Guerrieri, Marco

Guevara Lopez, Pedro

Gui, Yonghao

Guida, Giulia

Guida, Marco
Guijarro, Francisco

Guilbert, Damien

Guilizzoni, Roberta

Guillaud, Xavier

Guinjoan Gispert, Francesc

Guirao, Begoña

Gul, Mehreen

Gulkowski, Slawomir

Gullo, Paride

Gullon, Beatriz

Gullon, Patricia

Gumbyte, Milda

Gunasekara, Saman

Gunasooriya, G. T. Kasun Kalhara

Gunawardana, Upul

Guo, Boyun

Guo, Brian

Guo, Dejun

Guo, Jingli

Guo, Jinhua

Guo, Qiandong

Guo, Siwei

Guo, Wei

Guo, Xiangfeng

Guo, Xiaofeng

Guo, Youguang

Guo, Zhanjun

Gupta, Ipsita

Gupta, Pragya Kirti

Gupta, Shubhangi

Gurau, Vladimir

Gurgenci, Hal

Gurung, Thulo Ram

Gusev, Michail

Gu-Stoppel, Shanshan

Gutiérrez, Álvaro

Gutiérrez, Claudia

Gutiérrez, Roberto

Gutiérrez-Avilés, David

Gutiérrez-Martín, Fernando

Guziałowska-Tic, Joanna

Guzik, Maciej

Guzmán, Eduardo

Guzman, Marcelo

Guzzini, Alessandro

Gwendoline, Christophe

Gwozdz, Michal

Gwozdziewicz, Maciej

Gyore, Attila

Ha, Dong Sam

Ha, Heung Yong 
Haar, Lawrence

Habek, Patrycja

Haben, Stephen

Haber, Rodolfo

Häber, Thomas

Habib, Hany

Habibi, Ali

Habibi, Hamed

Habibi, Shahryar

Habrych, Marcin

Habuda-Stanic, Mirna

Haces Fernandez, Francisco

Hacker, Viktor

Hadad, Yossi

Hadas, Zdenek

Haddad, Abderrahmane

Haddad, Diego Barreto

Hadi, Pradita

Hadjadj, Aomar

Hadjistassou, Constantinos

Haerinia, Mohammad

Hafezi, Hossein

Hagar, Mohamed

Hagem, Rabee

Haghbin, Saeid

Haghighi, Arman

Hagishima, Aya

Hagiwara, Manabu

Hagner, Marleena

Haider, Azad

Haider, Noman

Hailu, Getu

Hajduga, Arkadiusz

Hajej, Zied

Hajilou, Mohammadhadi

Hajiyev, Natig Qadim-Oglu

Hajizadeh, Amin

Hakula, Harri

Halawa, Edward

Haldi, Frédéric

Hale, Rip

Haleem, Naushath M.

Halicioglu, Ferda

Halicka, Katarzyna

Halim, Md Abdul

Haliniarz, Małgorzata

Halinka, Adrian

Halisch, Matthias

Halkos, George

Hall, Carrie

Hall, Joshua
Hall, Monika

Haller, Alina

Hallinan, Kevin

Halm, Damien

Hałon, Tomasz

Halunga, Simona Viorica

Hamadani, Behrang

Hamanaka, Akihiro

Hamawand, Ihsan

Hamdani, Ari

Hamdi, Hamidreza

Hamed, Ali M.

Hameed, Saqib

Hamilton, Nicholas

Hammadi, Moncef

Hammarström, Thomas

Hammerstrom, Donald J.

Hamori, Shigeyuki

Han, Jeong Min

Han, Kunjun

Han, Kyoungseok

Han, Mengjie

Han, Sangwook

Han, Sekyung

Han, Soohee

Han, Weiji

Han, Won-Sik

Han, Yongming

Hanaoka, Toshiaki

Hand, Jon

Handayani, Kamia

Hangos, Katalin M.

Hanif, Asad

Hanif, Muhammad

Hanifi, Hamed

Hann, Christopher

Hann, Veryan Anastasia Joan

Hannan, Mohammed Abdul

Hansen, Clifford W.

Hanzelka, Zbigniew

Hao, Yong

Haoyuan, Hong

Haq, Anwar Ul

Haq, MuhammadAziz Ul

Haque, Abu Niyam M. M.

Harahap, Charles Ronald

Harangi-Rákos, Mónika

Harangozo, Gábor

Hardenbicker, Ulrike

Hardie, Mary

Hardy, Adam 
Hardy, Tomasz

Hare, Colin

Hariharan, Deivanayagam

Harirchian, Ehsan

Hariri, Diako

Hariri-Ardebili, Mohammad Amin

Harja, Maria

Harley, Joel B.

Harlow, John

Haroun, Reham

Harper, Gavin

Harries, David

Harrington, Julie

Harris, Daniel

Harrisson, Simon

Hart, Kyle

Hartley, Damon

Hartmann, Christoph

Hartvigsson, Elias

Haruki, Mitsuru

Hasan, Ahmed

Hasan, Faruque

Hasan, Md Mubashwar

Hasan, Reaz

Hasanspahić, Nermin

Hasegawa, Makoto

Haseli, Yousef

Haseltalab, Ali

Haseneder, Roland

Hashemi, Saeed

Hashemi, Shervin

Hashizume, Yoichiro

Haslam, Andrew

Hasmatuchi, Vlad

Hassan, Abdelrahman $\mathrm{H}$.

Hassan, Mahmoud

Hassan, Marwa

Hassan, Sammer-UI

Hassanalian, Mostafa

Hassanein, Amro

Hassani, Hossein

Hassanin, Hany

Hassanpouryouzband, Aliakbar

Hassanzadeh-Barforoushi, Amin

Hata, Hiroo

Hatiegan, Cornel

Hatziargyriou, Nikos D.

Havelka, Juraj

Hawash, Zafer

Hawila, Abed Al Waheed

Hawkins, Adam J.
Haworth, Kristina M.

Hayajneh, Hassan S.

Hayami, Hitoshi

Hayati, Farzad

Haydary, Juma

Hayward, Jennifer A.

Haywood, John Stephen

Hazari, Md. Rifat

Hazra, Dibyendu

$\mathrm{He}$, Bao-Jie

$\mathrm{He}$, Chengyuan

He, Guanming

He, Jian

He, Nafei

He, Peiwen

$\mathrm{He}$, Xia

He, Xiaoyi

$\mathrm{He}$, Ximin

He, Yingdong

He, Yingxin

He, Zhouying

Healy, William M.

Heberle, Florian

Hebner, Robert

Hedvičáková, Martina

Heere, Michael

Hefele, Peter

Heffernan, Emma

Hegedűs, László

Hegler, Sebastian

Heibati, Seyedmohammadreza

Heidari, Hamidreza

Heidarinejad, Mohammad

Heidarpour Shahrezaei, Iman

Heiderhoff, Ralf

Heim, Dariusz

Heinlein, Reinhold

Heinonen-Tanski, Helvi

Heinz, Erbe

Heinze, Christian

Heinze, Thomas

Helenbrook, Brian

Helistö, Niina

Hellmuth, Olaf

Helmers, Eckard

Helseth, Arild

Hemes, Susanne

Hemmati, Arman

Hemmes, Kas

Hemmingsen, Tor

Hemraz, Usha D. 
Henao, Nilson

Henderson, Alan D.

Hendrick, Patrick

Hendrix, Marcel A. M.

Henein, Naeim

Henke, Markus

Henriksson, Malin

Henriques, Carla Oliveira

Henriques, Joao

Henry, Daniel

Henshall, Paul

Henzel, Maciej

Hepburn, Donald

Heras, Francisco

Herath, H. M. Ayala S.

Herder, Paulien

Herdzik, Jerzy

Herencsar, Norbert

Herghiligiu, Ionut Viorel

Herisanu, Nicolae

Herman, Emilia

Herman, Grigore

Hermanns, Miguel

Hermansen, John Erik

Hermoso-Orzáez, Manuel Jesús

Hernández, César

Hernandez, J. C.

Hernández-Callejo, Luis

Hernandez-Escobedo, Quetzalcoatl

Hernández-Jiménez, Fernando

Hernandez-Mejia, Jean Carlos

Hernández-Minguillón, Rufino J.

Hernández-Pérez, Iván

Hernes, Marcin

Hernik, Bartłomiej

Herrando, María

Herrera, Miguel Angel

Herrera, Rafael

Herrera-May, Agustin L.

Herrera-Orozco, Israel

Herrero, Juan Manuel

Herrero, María Josefa

Herrero-de Lucas, Luis Carlos

Herreros, José Martín

Herres, Gerhard

Herrmann, Christiane

Herrmann, Frank

Heshmati-alamdari, Shahab

Hester, Keith

Heubner, Christian

Heurtebise, Jean Yves
Heusinger, Jannik

Heuss-Aßbichler, Soraya

Heydari Gharahcheshmeh, Meysam

Heydari, Azim

Heydari, Rasool

Heydari, Shoeib

Heydari-doostabad, Hamed

Heymann, Fabian

Heyne, Joshua

Hidaka, Kishio

Hidalgo Herrador, José Miguel

Hidalgo-Betanzos, Juan María

Hidy, George

Hiernaux, Stéphane

Hiete, Michael

Higashikawa, Kohei

Higinbotham, Douglas

Hijikata, Yasuto

Hijosa-Valsero, María

Hilairet, Mickael

Hilfer, Michael

Hill, Aaron J.

Hillis, Andrew J.

Hilloulin, Benoit

Hilpert, Simon

Himpe, Christian

Hincapie, Rafael E.

Hinov, Nikolay

Hinz, Hartmut

Hirayama, Yukari

Hirose, Kei

Hirotaka, Takano

Hirsch, Matías

Hirz, Mario

Hitch, Michael

Hittinger, Eric

Hiziroglu, Huseyin

Hiziroglu, Salim

Hjertager, Bjørn

Hlava, Jaroslav

Hlaváč, Libor M.

Hlaváčová, Irena M.

Hloch, Sergej

Hlosta, Jakub

Hnatiuc, Mihaela

Ho, Carr

Ho, Chii-dong

Ho, Jörg

Hoang, Khoa Dang

Hoang, Nam V.

Hoang, Nguyen Tien 
Hoarau, Yannick

Hobara, Yasuhide

Hoblos, Ghaleb

Hočevar, Brigita

Hocko, Marian

Hodaka, Ichijo

Hodge, Harrison

Hoecker-Martinez, Martin

Hoes, Pieter Jan

Hoff, Craig J.

Hoffmann, Daniel

Hofmann, Lutz

Hogan, Rita

Hogan, William W.

Höger, Marek

Hohmann, Sören

Hoicka, Christina E.

Hojabri, Mojgan

Holderbaum, William

Holderer, Olaf

Holec, Josef

Holmberg, Henrik

Holmes, Ralph

Holstenkamp, Lars

Holubcik, Michal

Holubec, Viktor

Holubnyak, Yevhen

Holze, Rudolf

Hong, Boe-Shong

Hong, Jin-Long

Hong, Jongin

Hong, Junho

Hong, Min

Hong, Seung Ho

Hong, Sugwon

Hong, Tao

Hong, Tianqi

Hong, Tianzhen

Hong, Wei-Chiang

Hong, Ying-Yi

Hong, Youn-Sik

Hönig, Vladimír

Honus, Stanislav

Hooftman, Nils

Hoogsteen, Gerwin

Hooper, Jim

Hooper, Justin B.

Hooper, Peter

Hopf, Konstantin

Hopkins, Brandon James

Hopper, Richard
Horabik, Józef

Horák, Jakub

Horisawa, Sakae

Horita, Hiroki

Horla, Dariusz

Hornak, Jaroslav

Horňák, Peter

Horne, Robert James

Horng, Shih-Cheng

Horrocks, Tom

Horttanainen, Mika

Horváth, Ilona Sárvári

Horváth, Krisztián

Horváth, Miklós

Hosein, Ian Dean

Hossain, Abul

Hossain, Akter

Hossain, Mamdud

Hossain, Md Alamgir

Hossain, Md Ashraf

Hossain, Md Moinul

Hossain, Md Sazzad

Hossain, Md. Alamgir

Hossain, Md. Zakaria

Hossain, Mofazzal

Hossain, Mohataz

Hossain, Nazia

Hossein Mardi, Ali

Hosseini, Matin

Hosseini, Seyed Ehsan

Hosseini, Seyed Saeid

Hosseinzadeh, Mehdi

Hosseinzadeh, Nasser

Hostettler, Silvia

Hoteit, Hussein

Hou, Jason

Hou, Ping

Houari, Azeddine

Houde, Sébastien

Houska, Jiri

Hovde, Blake T.

Howell, Bob

Hower, James

Hoyos Velasco, Fredy

Hożejowska, Sylwia

Hoznedl, Michal

Hożyń, Stanisław

Hraska, Jozef

Hrdlicka, Frantisek

Hrdlicka, Jan

Hredzak, Branislav 
Hren, Alenka

Hristova, Snezhana

Hroncová, Emília

Hruska, Karel

Hrzina, Pavel

Hsiao, Fu-yuen

Hsiao, Kai Long

Hsieh, Bieng-Zih

Hsieh, Hsin-Hsin

Hsieh, Jui-Ching

Hsieh, Yao-Ching

Hsu, Hsin-Wei

Hsu, Hung-Chu

Hsu, Ming-wei

Hsu, Yuan-Yih

Hsu, Yu-Kuei

$\mathrm{Hu}$, Aixue

$\mathrm{Hu}$, Changjian

$\mathrm{Hu}$, Changwei

$\mathrm{Hu}$, Gang

$\mathrm{Hu}$, Guoping

$\mathrm{Hu}$, Howard H.

$\mathrm{Hu}$, Jianan

$\mathrm{Hu}$, Jianjun

$\mathrm{Hu}$, Jin-Li

$\mathrm{Hu}$, Maomao

$\mathrm{Hu}$, Ming

$\mathrm{Hu}$, Xiaosong

$\mathrm{Hu}$, Yukun

Hua, Rong

Hua, Wei

Huang, Chao

Huang, Chao-Ming

Huang, Chia-Hua

Huang, Chih-Fang

Huang, Chung-Chi

Huang, Chung-Neng

Huang, Gan

Huang, Gordon

Huang, Haiyan

Huang, Hsin-Haou

Huang, Jenny

Huang, Jih-Jeng

Huang, Jing

Huang, Kevin

Huang, Ko-Wei

Huang, Kuo-Tsang

Huang, Nantian

Huang, Pei

Huang, Peihua

Huang, Peipei
Huang, Ping

Huang, Sen

Huang, Sunan

Huang, Tzu-Yen

Huang, Weimin

Huang, Wei-Tzer

Huang, Wen Chang

Huang, Wenjing

Huang, Wu-Jang

Huang, Xiaoxue

Huang, Yu-Hsi

Huba, Mikuláš

Hubačíková, Věra

Hubenova, Yolina

Huber, Julian

Huber, Miłosz

Hübner, Renate

Hudişteanu, Valeriu Sebastian

Huerta Sánchez, Francisco

Huerta, Nicolas

Huertas, Rosa

Hughes, Tom

Hugi, Christoph

Huh, Chun

Huh, Jun-Ho

Huh, Taewook

Hui, Sam C. M.

Hui, Teo Tee

Hulme-Smith, Christopher Neil

Humar, Miha

Humelnicu, Costel

Huminic, Gabriela

Hummel, Karin Anna

Humphries, Terry

Hung, Chia-Hung

Hung, Nguyen Tuan

Hunicz, Jacek

Huo, Jiajie

Huo, Yiming

Huot, Jacques

Hupez, Martin

Huque, Ziaul

Hur, Deog Jae

Hur, Jin

Hur, Kyeon

Hure, Nikola

Hurnik, Maria

Hursin, Mathieu

Hurth, Victoria

Husev, Oleksandr

Husmann, Anke 
Hussain Nengroo, Sarvar

Hussain, Amjad

Hussain, Babar

Hussain, Essam

Hussain, Ghulam Amjad

Hussain, Majid

Hussain, Muhammad Imtiaz

Hussain, Muhammad Majid

Hussain, Niamat

Hussain, Sajjad

Hussain, Shahid

Hussein, Hussam

Huu Nguyen, Trung

Huynh, Van Khang

Huzlik, Rostislav

Hvelplund, Frede

Hwang, Eenjun

Hwang, Hsiu-Ying

Hwang, Myun Joong

Hwang, Pyeong-Ik

Hwang, Seon-Hwan

Hwang, Young Jin

Hwangbo, Hyunwoo

Hwu, Kuo-Ing

Hybská, Helena

Hyhlík, Tomáš

Hytiris, Nicholas

Hyun, Seung-Ho

Iacobucci, Riccardo

Ianakiev, Anton

Iancovici, Mihail

Ianniello, Roberto

Ianole-Calin, Rodica

Iantovics, Laszlo Barna

Iasiello, Marcello

Iba, Chiemi

Ibáñez Mendizábal, Raquel

Ibáñez, Santiago E.

Ibarra, David

Ibarra-Berastegi, Gabriel

Ibrahim, Hussameldin

Ibrahim, Mohamed Nabil Fathy

Ibrahimi, Abdul Matin

Iclodean, Calin

Idehen, Ikponmwosa

Idsø, Johannes

Idzkowski, Adam

Iero, Demetrio

Ieropoulos, Ioannis A.

Ifelebuegu, Augustine Osamor

Igathinathane, Cannayen
Iglauer, Stefan

Ignat, Maria

Igreja, José

Igual, Jorge

Ihara, Takeshi

Ihsanullah, Ihsanullah

Iio, Jun

lioka, Daisuke

Ijaz, Muhammad Fazal

Ikegami, Masashi

Ikegaya, Naoki

Ikeo, Shigeru

Ikhide, Monday

Ikram, Awais

Ilak, Perica

Ileana Corcau, Jenica

Ileš, Šandor

Ilic-Stojanovic, Snezana

Ilie, Constantin

Ilie, Marcel

Ilinca, Adrian

Ilinčić, Petar

Ilinova, Alina

Ilo, Albana

Ilomäki, Jukka

Iluk, Artur

Ilves, Risto

Im, Piljae

Im, Seong-Kyun

Imburgia, Antonino

Imperatore, Stefania

Imran, Muhammad

Imre, Attila R.

Imtiaz, Masudul Haider

Inaba, Atsushi

Inagaki, Atsushi

Inard, Christian

Incremona, Gian Paolo

Inderberg, Tor-håkon

Indrawan, Natarianto

Ingale, Nilesh

Ingber, Lester

Inhestern, Lukas Benjamin

Inokuchi, Tsutomu

Introna, Vito

Inukai, Junji

Ioakimidis, Christos

Ioannides, Theophilos

Ioannou, Konstantinos

Ioannou, Stelios

Iodice, Paolo 


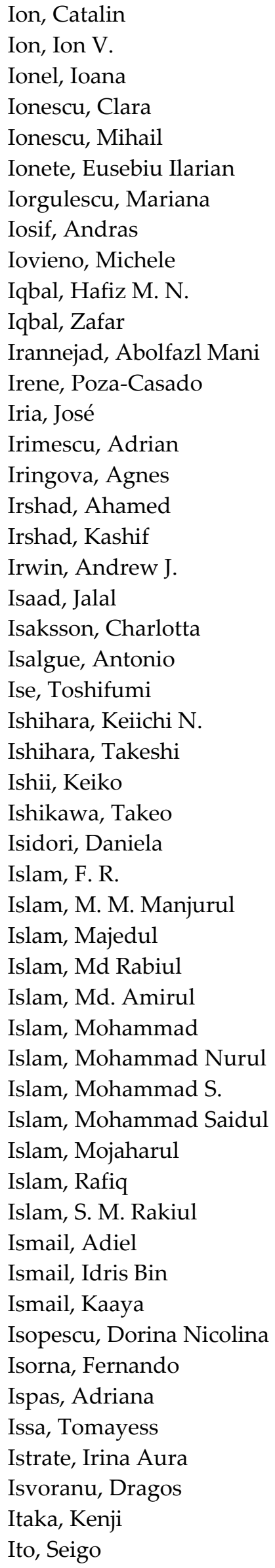

\author{
Ito, Tadashi \\ Ityokumbul, M. Thaddeus \\ Iudice, Ivan \\ Iulia-Alina, Anton \\ Iulianelli, Adolfo \\ Ivančić Šantek, Mirela \\ Ivanescu, Mircea \\ Ivanković, Igor \\ Ivannikov, Alexander \\ Ivanov, Ovidiu \\ Ivanov, Roman V. \\ Ivanov, Toni \\ Ivanov, Valentin \\ Ivanov, Vladimir K. \\ Ivanova, Diana \\ Ivanovich, Tyurin Yury \\ Ivascu, Larisa \\ Ivšinović, Josip \\ Iwafune, Yumiko \\ Iwan, Agnieszka \\ Iwaniszyn, Marzena \\ Iwanski, Grzegorz \\ Iwaszkiewicz, Jan \\ Iwata, Shinya \\ Iyer, Lakshmi Varaha \\ Izadgoshasb, Iman \\ Izbassarov, Daulet \\ Izydorczyk, Jacek \\ Jabbari, Faryar \\ Jabbari, Hadi \\ Jaber, Mona \\ Jaberg, Helmut \\ Jabłoński, Adam \\ Jabłoński, Paweł \\ Jacak, Janusz \\ Jack, Michael W. \\ Jackowski, Mateusz \\ Jacob, Isaac \\ Jacob, Paul \\ Jacobs, David E. \\ Jacobs, Jan P. A. M. \\ Jacques, Sebastien \\ Jacquod, Philippe \\ Jąderko, Andrzej \\ Jadhav, Shrikant \\ Jadhawar, Prashant \\ Jadidi, Mehdi \\ Jadlovska, Slavka \\ Jadwiszczak, Piotr \\ Jaeckel, Bengt \\ Jaen Sola, Pablo
}




\author{
Jafari Kaleybar, Hamed \\ Jafari, Mohammad (Australia) \\ Jafari, Mohammad (USA) \\ Jafari, Raheleh \\ Jafari, Soheil \\ Jafarinejad, Shahryar \\ Jafri, Yawer \\ Jägerbrand, Annika K. \\ Jäger-Waldau, Arnulf \\ Jagodziński, Jacek \\ Jagtap, Sujit \\ Jahanbakhsh, Amir \\ Jahanbin, Aminhossein \\ Jahromi, Hossein \\ Jaime, Zapata \\ Jain, Ankur \\ Jain, Himanshu \\ Jaiswal, Priyank \\ Jajczyk, Jarosław \\ Jakica, Nebojsa \\ Jakovac, Pavle \\ Jaksic, Vesna \\ Jakub, Čedík \\ Jakubowski, Marek \\ Jakubowski, Robert \\ Jakus, Damir \\ Jalakas, Tanel \\ Jalali, Mandana \\ Jaliu, Codruta \\ Jamal, Taskin \\ Jamalabadi, Mohammad Yaghoub \\ Abdollahzadeh \\ James, Corey M. \\ James, Geoffrey \\ James, Patrick \\ James, Sagil \\ Jamrozik, Arkadiusz \\ Jamshidi, Reihaneh \\ Jamshidpour, Ehsan \\ Janas, Dawid \\ Janczura, Joanna \\ Janda, Tomas \\ Janecki, Ryszard \\ Jang, Seon-Jun \\ Jangi, Mehdi \\ Janicka, Anna \\ Janicki, Marcin \\ Janiga, Damian \\ Janik, Przemyslaw \\ Janiszewski, Dariusz \\ Janiszewski, Mateusz
}

Janjic, Aleksandar

Janko, Samantha A.

Jankowska-Mihułowicz, Marzena

Jankowski, Marcin

Jankowski, Piotr

János, Szanyi

Janošovský, Ján

Janot, Raphael

Janous, Stepan

Janovec, Michal

Janssen, Hans

Jansto, Steven G.

Janus, Rafał

Janus, Thorsten

Janusas, Giedrius

Januševičius, Karolis

Jaramillo-Morán, Miguel A.

Jarczewski, Sebastian

Jaremkiewicz, Magdalena

Jareño, Francisco

Jaroslav, Vrchota

Jaroszewski, Maciej

Jaroszuk-Ściseł, Jolanta

Jarquin Laguna, Antonio

Jarzebowicz, Leszek

Jarzebski, Maciej

Jarzyna, Jadwiga A.

Jarzyna, Wojciech

Jasa, John

Jasieniak, Jacek

Jasińska, Elżbieta

Jasiński, Mariusz

Jasinski, Michal

Jasiński, Tomasz

Jasiulewicz, Malgorzata

Jasiunas, Lukas

Jaskiewicz, Marek

Jaskólski, Marcin

Jastrzebska, Agnieszka

Jastrzębska, Małgorzata

Jauhiainen, Jussi

Javadi, Shahram

Javaid, Saher

Javanroodi, Kavan

Javed, Saqib

Javid, Roxana J.

Javorski Eckert, Jony

Jawad, Ghulam

Jaworek, Anatol

Jaworski, Maciej 
Jayachandran, K. P.

Jayakumar, Arunkumar

Jayaraman, Sundaramurthy

Jayathurathnage, Prasad

Jayatissa, Ahalapitiya H.

Jayne, Richard

Jechow, Andreas

Jeddi, Babak

Jedynak, Katarzyna

Jee, Solkeun

Jeffs, James

Jefimowski, Wlodzimierz

Jegla, Zdenek

Jelemenský, L'udovít

Jeleński, Tomasz

Jelley, Nick

Jemcov, Aleksandar

Jenčová, Edina

Jendruś, Rafał

Jendryś, Marek

Jensen, Ole MIchael

Jensen, Per Anker

Jeong, Chang Kyu

Jeong, Hyun-dam

Jeong, Jongryeol

Jeong, Soo Jin

Jeong, Yeonho

Jereb, Borut

Jeremiáš, Michal

Jerković Štil, Vedrana

Jerzak, Wojciech

Jesenik, Marko

Jeske, Tim

Jesus, Isabel

Jewell, Eifion Huw

Jewiarz, Marcin

Jezierski, Jan

Jezowski, Pawel

Jha, Birendra

Jha, Nand

Jha, Pankaj

Jheng, Li-Cheng

Ji, Dongsheng

Ji, Dongxu

Ji, Jinchen

Jia, Bao

Jia, Boru

Jia, Charles

Jia, Guanbo

Jia, Junbo

Jia, Mengda
Jia, Wei

Jia, Xingyun

Jia, Yu

Jia, Yunyan Andrea

Jiang, Chengmin

Jiang, Jieke

Jiang, Jinwei

Jiang, Junke

Jiang, Liangliang

Jiang, Lin

Jiang, Long

Jiang, Qiu

Jiang, Ruinian

Jiang, Shan

Jiang, Wei

Jiang, Yajie

Jiang, Yeu-Long

Jiang, Zhiyu

Jiao, Kui

Jiao, Yan

Jibrin, Abubakar Abbas

Jicsinszky, László

Jige, Daisuke

Jimenez Alonso, Javier Fernando

Jiménez Millán, Juan

Jimenez, Emilio

Jiménez, Felipe

Jiménez, Jorge Cara

Jimenez-Bescos, Carlos

Jiménez-Bravo, Diego M.

Jiménez-Buendía, Francisco

Jiménez-Espadafor, Francisco José

Jimenez-Hornero, Jorge E.

Jin, Jiangliang

Jin, Li

Jin, Wencheng

Jin, Yaqing

Jin, Young Gyu

Jin, Zhiyang

Jindo, Keiji

Jing, Qingshen

Jing, Xia

Jing, Xingjian

Jirásek, Jakub

Jirásko, Daniel

Jirout, Tomas

Jiu, Jinting

Jnr, Bokolo Anthony

Jo, Sae Byeok

Jo, Young Min

Jodar-Abellan, Antonio 


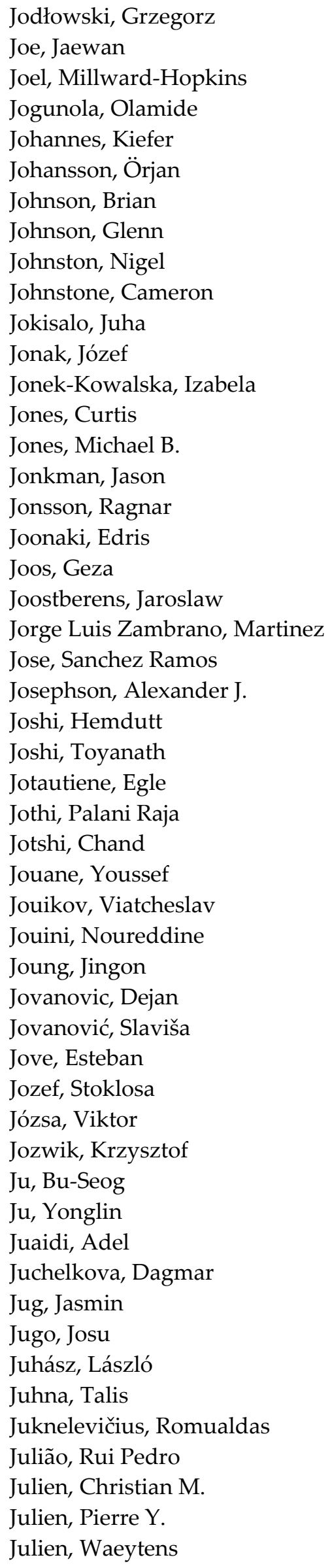

Jun, Sunghae

Jun, Yongseok

Jun, Young-Si

Jung, Christopher

Jung, Heejung

Jung, Kyung Hwan

Jung, Kyung-Hye

Jung, Minsu

Jung, Seok-Ki

Jung, Sokhee Philemon

Jung, Sungdo

Jung, Sunghun

Jung, Sung-Moon

Jung, Wooyong

Jung, Young Hoon

Junga, Robert

Junghans, Lars

Junker, Rune Grønborg

Junyent-Ferré, Adrià

Juodkazytè, Jurga

Jurado, Maria Jose

Juráš, Peter

Jurasz, Jakub

Jurca, Florin Nicolae

Jurdziak, Leszek

Jureczko, Mariola

Jūrènas, Vytautas

Jurić-Grgić, Ivica

Juričić, Đani

Jurić-Kaćunić, Danijela

Jurišić, Dražen

Jusas, Vacius

Kaabouch, Naima

Kaario, Ossi

Kabalyk, Kirill

Kabošová, Lenka

Kacejko, Piotr

Kacem, Najib

Kačerauskas, Tomas

Kachniarz, Marian

Kacor, Petr

Kačur, Ján

Kaczmarczyk, Michał

Kaczmarek, Michal

Kadam, Avinash

Kadapa, Chennakesava

Kádár, Csilla

Kadar, Peter

Kadela, Marta

Kadirova, Seher

Kadokawa, Jun-ichi 
Kaewunruen, Sakdirat

Kagan, Lonid

Kagemoto, Hiroshi

Kagkoura, Antonia

Kahl, Annelen

Kahrobaee, Salman

Kairytè, Agnè

Kaiwartya, Omprakash

Kajanova, Martina

Kajjam, Aravind

Kakanakov, Nikolay

Kakarountas, Athanasios (Thanos)

Kakati, Nitul

Kaklis, Kostas

Kalagasidis, Angela Sasic

Kalaitzidis, Stavros

Kalambura, Sanja

Kalangala Sikkanther Batcha, Syed Ali

Kalderis, Dimitrios

Kaledina, Nina O.

Kaletova, Tatiana

Kalhor, Elmira

Kalibatiene, Diana

Kalina, Martin

Kalinichenko, Antonina

Kalisz, Sylwester

Kalivoda, Jan

Kaliyan, Nalladurai

Kallaste, Ants

Kallert, Anna

Kalligeros, Stamatis Spyridon

Kallweit, Stephan

Kalmar, Ferenc

Kalmár, József

Kalnaus, Sergiy

Kalogirou, Soteris

Kaltenbach, Hans-Jakob

Kaltschmitt, Martin

Kaluža, Luděk

Kamachi, Toshiaki

Kamal, Rajeev

Kamaltdinov, Vyacheslav

Kamatham, Nareshbabu

Kamberović, Željko

Kambezidis, Harry

Kambiz, Kambiz

Kambiz, Tehrani

Kamel, Raghad

Kamguia Simeu, Severin

Kaminska, Aniela

Kamińska-Benmechernene, Aniela
Kaminski, Daniel

Kaminski, Jacek

Kaminski, Marcin

Kampczyk, Arkadiusz

Kampelis, Nikos

Kamperidou, Vasiliki

Kamrat, Waldemar

Kamzolova, Svetlana

Kanakaris, Venetis

Kanakoudis, Vasilis

Kanamarlapudi, Venkata Ravi Kishore

Kanapickiene, Rasa

Kanarachos, Stratis

Kanbur, Barış Burak

Kancherla, Raghu

Kanda, Wisdom

Kandhasamy, Sathiyaraj

Kandil, Mohamed

Kaneda, Masayuki

Kaneko, Shozo

Kang, Daeho

Kang, Dong-Ki

Kang, Dong-Won

Kang, He-Yau

Kang, Jidong

Kang, Jimyung

Kang, Jun

Kang, Kyu

Kang, Lei

Kang, Ning

Kang, Sang Hoon

Kang, Seok Ju

Kang, Seongmin

Kang, Song-Lak

Kang, Sung Jin

Kang, Sung-Hoon

Kang, Xin

Kangas, Hanna-Liisa

Kania, Aneta

Kania, Dariusz

Kaniewski, Jacek

Kannan, Ravi

Kanniche, Mohamed

Kant Bhatia, Shashi

Kant, Krishan

Kantar, Emre

Kantartzis, Nikolaos V.

Kantor, Ivan Daniel

Kantzas, Apostolos

Kapasakalis, Konstantinos

Kapcia, Konrad Jerzy 
Kapetanakis, Theodoros N.

Kapica, Jacek

Kaplon, Andrzej

Kaplun, Dmitrii

Kapogiannis, Georgios

Kappatou, Joya C.

Kapranov, Sergey

Kapsalis, Vasilios C.

Kapteijn, Freek

Kapusta, Kyzysztof

Kapusta, Łukasz Jan

Kapustin, Ivan A.

Kar, Anuradha

Kar, Samarjit

Kara, Kursat

Karabasevic, Darjan

Karabasov, Sergey

Karaivanov, Dimitar

Karakašić, Mirko

Karakasidis, Theodoros

Karakatič, Sašo

Karamov, Dmitriy

Karampour, Hassan

Karanasios, Konstantinos

Karanayil, Baburaj

Karanikas, Nektarios

Karasali, Helen

Karasalihović-Sedlar, Daria

Karásek, Matěj

Karaśkiewicz, Krzysztof

Karathanasis, Stavros

Karathanassis, Ioannis

Karavas, Christos-Spyridon

Karayaka, Bora

Karažija, Tomislav

Karbalaei Baba, Alireza

Karbuz, Sohbet

Karczewski, Grzegorz

Kardas, Dariusz

Karie, Nickson M.

Karim, M. A.

Karimi, Nader

Karimkashi, Shervin

Karlen, Steven

Karlis, Athanasios

Karlsson, Hyunjoo

Karnama, Ahmad

Karnavas, Yannis

Karney, Bryan

Karni, Jacob

Karolinczak, Beata
Karoń, Grzegorz

Karpetis, Adonios

Karrar, Abdelrahman

Karthick, SK

Karthikeyan, Obulisamy Parthiba

Kartini, Unit Three

Kartopu, Giray

Kartsakli, Elli

Karuppasamy, K.

Karvounis, Artemios

Karvounis, Dimitrios

Karytsas, Spyridon

Kasa, Sudheer

Kasać, Josip

Kasbaoui, Mohamed

Kascak, Slavomir

Kashani, Hamzeh

Kashchenko, Ilia S.

Kashif, Muhammad

Kasina, Monika

Kasina, Saamrat

Kasiński, Krzysztof

Kasiulis, Egidijus

Kasmaee, Sara

Kasnatscheew, Johannes

Kasperski, Andrzej

Kasperski, Jacek

Kasprzyk, Leszek

Kassai, Miklos

Kästner, Christian

Kasymov, Denis

Kaszyński, Przemysław

Katerski, Atanas

Kathe, Mandar

Katić, Vladimir

Katin, Konstantin

Katioglu-Yazan, Tugce

Kato, Hironori

Kato, Yoshimine

Katona, Tamás János

Katrasnik, Tomaz

Katsaounis, Georgios M.

Katsigiannis, Yiannis

Katsikas, Sokratis

Katsoulas, Nikolaos

Katsura, Takao

Katunský, Dušan

Katz, Joseph

Katzschner, Lutz

Kauhaniemi, Kimmo

Kaushal, Vinayak 
Kavadias, Kosmas

Kavan, Ladislav

Kavgic, Miroslava

Kawa, Adam

Kawa, Arkadiusz

Kawakita, Hidetaka

Kawanabe, Hiroshi

Kaya, Alihan

Käyhkö, Jukka

Kazak, Andrey

Kazak, Jan

Kazakbaev, Vadim

Kazana, Vassiliki

Kazda, Tomáš

Kazemifar, Farzan

Kazemtabrizi, Behzad

Kazimierowicz, Joanna

Kazmi, Jawad

Kazmierczak, Andrzej R.

Kazmierkowski, Marian P.

$\mathrm{Ke}$, Yan

Keane, Andrew

Kearney, Paul

Kechagias, John D.

Kehagia, Fotini

Keip, Marc-Andre

Keivanpour, Samira

Kelarakis, Antonios

Kelemen, Miroslav

Keller, Hubert

Keller, Joerg

Kelley, Douglas H.

Kenea, Samuel Takele

Kepplinger, Peter

Keramatikerman, Mahdi

Kerdphol, Thongchart

Kerekes, Tamás

Kermani, Mostafa

Kermanshah, Amirhassan

Keshavarz, Alireza

Keshavarzian, Sajjad

Kezirian, Michael T.

Khabaz, Fardin

Khadem, Shafiuzzaman K.

Khademian, Zoheir

Khadka, Dhruba B.

Khaledialidusti, Rasoul

Khalghani, Mohammad Reza

Khalid, Farrukh

Khalifeh, Mahmoud

Khalili, Siavash
Khalilpour, Kaveh

Khaliq, Jibran

Khambadkone, Ashwin M.

Khamkhash, Aibyek

Khan, Arifur R.

Khan, Ashraf Ali

Khan, Danish

Khan, Imran

Khan, Inayat Ali

Khan, Irfan

Khan, Md. Shakhaoath

Khan, Mohammad

Khan, Mohammad Ehtisham

Khan, Mohammed Nazeer

Khan, Muhammad Omer

Khan, Muhammad Shuja

Khan, Saad Ullah

Khan, Sanowar

Khan, Sohail

Khan, Umar F.

Khan, Ziaudding

Khanal, Aaditya

Khanal, Rabi

Khandelwal, Manoj

Khanesar, Mojtaba Ahmadieh

Khani, Sina

Khanna, Sourav

Kharrat, Riyaz

Kharrazi, Ali

Khatib, Tamer

Khatibi, Arash

Khatir, Samir

Khatiwada, Dilip

Khatoon, Asma

Khattri, Sanjay Kumar

Khazraee, Milad

Khelf, Ilyes

Khersonsky, Yuri

Khishvand, Mahdi

Khlyustova, Anna

Khmelinskii, Igor

Khodabakhsh, Javad

Khodabakhshnejad, Arman

Khodadadi, Jay M.

Khojasteh, Danial

Khokle, Rajas Prakash

Khooban, Mohammad Hassan

Khorasany, Mohsen

Khormali, Shahab

Khoshmanesh, Khashayar

Khoshsirat, Nima 
Khosravy, Mahdi

Khovalyg, Dolaana

Khovanov, Igor

Khura, Maninder

Khurshiad, Tahir

Khurshid, Chrow

Khushalani Solanki, Sarika

Kiausiene, Ilona

Kibria, Md. Golam

Kic, Pavel

Kichou, Sofiane

Kicinski, Marcin

Kida, Masato

Kiefer, Christoph Patrick

Kies, Alexander

Kiesewetter, Dmitry

Kii, Masanobu

Kiipli, Tarmo

Kijo-Kleczkowska, Agnieszka

Kikas, Timo

Kikkert, Cornelis

Kikugawa, Gota

Kikusato, Hiroshi

Kil, Gyung-Suk

Kilby, Jeffrey

Kilikevičienė, Kristina

Kilkovský, Bohuslav

Killian, Manuela Sonja

Kim, Aerhan

Kim, Albert

Kim, Bom Soo

Kim, Byungtaek

Kim, Chang Ki

Kim, Chan-Ki

Kim, Chunghun

Kim, Daehwan

Kim, Daehyeon

Kim, Daeyoung

Kim, Do Hyun

Kim, Dong Hoon

Kim, Dong Kyu

Kim, Dongwook

Kim, Eel-Hwan

Kim, Eui Jong

Kim, Gyeong-Min

Kim, Hag-Wone

Kim, Hana

Kim, Hanki

Kim, Hansung

Kim, Hogyoung

Kim, Howon
Kim, Huy Kang

Kim, Hye-Jin

Kim, Hyoung-Ho

Kim, Hyun Goo

Kim, Hyun Jin

Kim, Jaehyun

Kim, Jaemin

Kim, Jang Mok

Kim, Jincheol

Kim, Jingook

Kim, Jin-Hyuk

Kim, Jinsoo

Kim, Jong-Chan

Kim, Jong-Soo

Kim, Jooyoun

Kim, Ju Hyung

Kim, Jung Kyu

Kim, Jungsoo

Kim, Jung-Su

Kim, Junkyeong

Kim, Ki-Chan

Kim, Ki-Du

Kim, Kwanho

Kim, Kyeong-Hwa

Kim, Kyu-Han

Kim, Man-Hoe

Kim, Min

Kim, Min Soo

Kim, Min Su

Kim, Minsung

Kim, Mun-Kyeom

Kim, Myungseob (Edward)

Kim, Myungsun

Kim, Sahm

Kim, Sang Moon

Kim, Sanghoon

Kim, Sanghyun

Kim, Sangtae

Kim, Sangwon

Kim, Seok-Hyun

Kim, Seok-Kyoon

KIM, Seon Tae

Kim, Seong-Kyu

Kim, Seoyong

Kim, Soaram

Kim, Sojung

Kim, Soo

Kim, Sung Suk

Kim, Sung Won

Kim, Sung-Min

Kim, Sun-Sook 
Kim, Taeyeon

Kim, Wonhee

Kim, Wookyung

Kim, Yonghee

Kim, Yong-Joo

Kim, Youngbae

Kim, Youngbok

Kim, Young-Chon

Kim, Youngchul

Kim, Young-Joo

Kim, Young-Joon

Kim, Young-Ju

Kim, Young-Kee

Kim, Youngmin

Kim, Youngwoo

Kimoto, Sayuri

Kinoshita, Hiroyuki

Kinuthia, John

Kiourt, Chairi

Kiraga, Marta

Kiran, Raj

Kircher, Manfred

Kirei, Botond Sandor

Kirichenko, Ksenia

Kirilov, Plamen

Kirkham, Sandra

Kirm, Marco

Kishore, Ravi Anant

Kisielewicz, Tomasz

Kisilewicz, Tomasz

Kiss, István

Kiss, Laszlo I.

Kissai, Moad

Kistelegdi, István

Kisu, Kazuaki

Kitahara, Tatsumi

Kitajou, Ayuko

Kitak, Peter

Kitanovski, Andrej

Kitsios, Fotis

Kiwata, Takahiro

Kizek, Ján

Klačková, Ivana

Klaić, Zvonimir

Klarecki, Klaudiusz

Klauson, Aleksander

Klavins, Maris

Klein, Susanne

Kleine-Möllhoff, Peter

Kleiner, Karin

Klemeš, Jiří Jaromír
Klempa, Martin

Klepacki, Dariusz

Klewicka, Elzbieta

Klika, Vaclav

Klimenda, František

Klimeš, Lubomír

Klimkowicz-Pawlas, Agnieszka

Klink, Kathy

Klishin, Sergey

Kljenak, Ivo

Kloker, Markus J.

Kłos, Marcin

Kłos, Mariusz

Klose, Andrew

Kłosowski, Grzegorz

Klucznik, Jacek

Kluger, Jocelyn M.

Klühspies, Johannes

Klumpner, Christian

Klusman, Ronald W.

Klüss, Joni

Klyuev, Roman V.

Kminiak, Richard

Knap, Vaclav

Knápek, Alexandr

Knapik, Ewa

Knehr, Kevin

Knez, Dariusz

Knežević, Goran

Knight, Christopher

Knight, Travis W.

Knights, Peter

Knittel, Dominique

Knockaert, Jos

Knutsson, Pavleta

Ko, Chia-Nan

Ko, Chun-Han

Ko, Jang Myoun

Ko, Kyungnam

Kobayashi, Hiroyasu

Kobayashi, Koichi

Kobayashi, Rika

Kobetičová, Klára

Koblischka, Michael R.

Kobrle, Pavel

Koch, Sascha

Koch, Wojciech

Kočí, Jan

Kočí, Vladimír

Kocian, Alexander

Kocifaj, Miroslav 
Kocsi, Balázs

Koda, Eugeniusz

Kodaira, Daisuke

Kodalle, Tim

Kodogiannis, Vassilis

Kodura, Apoloniusz

Koech, Phillip

Koenig, Gary

Koh, Katherine

Kohl, Holger

Köhler, Manfred

Kohli, Kirtika

Koirala, Binod

Koivisto, Matti Juhani

Koiwa, Kenta

Koji, Yamamoto

Kojima, Yoshiyuki

Kokkinos, Nikolaos C.

Kokko, Marika E.

Kokoura, Sofia

Kolar, Vaclav

Kolasińska, Ewa

Kolasiński, Piotr

Kolator, Bronisław Andrzej

Kolcun, Michal

Kolcunová, Iraida

Kolenda, Zygmunt

Kolev, Mikhail

Kolláth, Zoltán

Kölle, Konstanze

Kollmeyer, Phillip J.

Kolmes, Steven A.

Kolmickovs, Antons

Kołodziej, Janusz

Kolomvatsos, Kostas

Koltsaklis, Nikolaos

Kómar, Ladislav

Komatsu, Hiroyuki

Komęza, Krzysztof

Komljenovic, Dragan

Komninos, Neofytos

Komova, Oxana

Konar, Arkaprabha

Konarik, Roman

KONCSAG, Claudia Irina

Kondaveeti, Sanath

Kondinski, Aleksandar

Kondori, Javad

Konev, Alexander

Kong, Jaemin

Kong, Lingyun
Kong, Ran

Kong, Xiangrui

Kong, Xiangyu

Konieczny, Jaroslaw

König-Haagen, Andreas

Konigorski, Ulrich

Koniorczyk, Piotr

Konoplev, Ivan

Konstanciak, Anna

Konstantakopoulou, Foteini

Konstantakopoulou, Fotini

Konstantinidis, Efstathios

Konstantinou, Georgios

Kontargyri, Vassiliki

Kontis, Eleftherios O.

Kontoni, Denise-Penelope N.

Kontou, Eleftheria

Kontses, Anastasios

Koo, Bonchan

Koo, Bon-Yong

Koo, Jamin

Koo, Junemo

Koo, Weon Cheol

Kopel, Pavel

Kopitar, Dragana

Kopka, Ryszard

Kopnina, Helen

Koppaka, Anjaneyulu

Kordana-Obuch, Sabina

Kordestani, Mojtaba

Kordi, Behzad

Koreanschi, Andreea

Korenko, Maros

Korentz, Jacek

Kõrgesaar, Mihkel

Korjenic, Azra

Korka, Zoltan

Korlos, Apostolos

Kornél, Németh

Korniejenko, Kinga

Korobeynikov, S. M.

Korodi, Adrian

Koroteev, Dmitri

Korpyś, Mateusz

Korsbakken, Jan Ivar

Korzeniewski, Marek

Korzeniowski, Waldemar

Korzhov, Anton

Kos, Goran

Kosacka-Olejnik, Monika

Kosan, Tomas 
Kosar, Ali

Kosasih, Buyung

Koscak Kolin, Sonja

Koshkin, Sergiy

Koshy, Pramod

Košičiarová, Ingrida

Kos-Łabędowicz, Joanna

Kosman, Wojciech

Kośny, Marek

Kosowski, Krzysztof

Kostakis, Ioannis

Kostal, David

Kostolný, Igor

Kostopoulos, Georgios

Kostrzewski, Mariusz

Kostúr, Karol

Kostyła, Paweł

Koszalka, Grzegorz

Koszelnik, Piotr

Koszewnik, Andrzej

Kot, Anna

Kot, Sebastian

Kota, Krishna

Kótai, László

Kotak, Yash

Kotek, Martin

Koter, Stanislaw

Kotevska, Olivera

Kothandaraman, Jotheeswari

Kotobuki, Masashi

Kotsampopoulos, Panos

Kotsiantis, Sotiris

Kott, Joanna

Kotter, Richard

Kotyra, Andrzej

Kotzur, Leander

Koubaa, Ahmed

Koubiti, Mohammed

Koudelková, Zuzana

Kouhshahi, Mojtaba Bahrami

Koukouvinis, Foivos (Phoevos)

Koukouzas, Nikolaos

Koulocheris, Dimitrios

Kouloumpis, Victor

Kourtis, Michael-Alexandros

Koutavarapu, Ravindranadh

Koutsandria, Georgia

Koutselas, Ioannis

Koutsos, Vasileios

Koutsovitis, Petros

Kouveliotis-Lysikatos, Iasonas
Kouziokas, Georgios N.

Kouzu, Masato

Kováč, Jaroslav

Kovač, Sanja

Kovacevic, Meho Sasa

Kovacevic-Badstuebner, Ivana

Kovacheva, Daniela

Kováčiková, Tatiana

Kovács, Róbert

Kovalcik, Adriana

Kovalov, Danyil

Kovář, Jiř́i

Kowal, Julia

Kowal, Przemysław

Kowalczyk-Juśko, Alina

Kowalik, Rafał

Kowalik, Ryszard

Kowalska, Ewa

Kowalska-Koczwara, Alicja

Kowalska-Pyzalska, Anna

Kowalski, Gregory

Kowalski, Zygmunt

Kowol, Marcin

Koyama, Michihisa

Kozai, Toyoki

Kozak, Maciej

Kozak, Miłosław

Kozarac, Darko

Kozhevnikov, Mikhail

Kozielski, Lucjan

Kozioł, Joachim

Kozioł, Michał

Kozioł, Piotr

Kozlov, Andrey

Kozlovskiy, Artem

Kozłowski, Edward

Kozulovic, Dragan

Kozyreff, Gregory

Krachkovskiy, Sergey

Kracík, Petr

Kraev, Gleb

Kraft, Michael

Kraft, Thomas

Kragović, Milan

Krah, Jens Onno

Krahn, Steven

Krainer, Ales

Kraj, Andrea

Krajcar, Slavko

Králik, Juraj

Kralj, Samo 
Krampe, Jörg

Kranjčević, Lado

Krantz, David

Krasiński, Adam

Kraus, Jakub

Kraus, Michal

Krause, Paweł

Krawczyk, Artur

Krawczyk, Dorota Anna

Krawczyk, Paweł

Krbal, Michal

Krcum, Maja

Krechowicz, Maria

Krejcar, Ondrej

Kremieniewski, Marcin

Krenz, Kimon

Krepel, Jiri

Krídlová Burdová, Eva

Krieger, Michael

Kriegseis, Jochen

Krishnamurthy, Raghavendra

Krishnamurthy, Vaidyanathan

Krishnaswamy, Bhuvana

Kristensen, Erik Fløjgaard

Kristiansen, Kai

Kristiansen, Tarjei

Kriston, Akos

Kröger, Wolfgang

Kroičs, Kaspars

Król, Aleksander

Król, Danuta

Król, Małgorzata

Krommydas, Konstantinos F.

Krop, Dave C. J.

Krstić, Hrvoje

Krukowski, Krzysztof

Krumdieck, Susan

Krümpel, Johannes

Krupa, Joel

Krupenev, Dmiry S.

Krupenski, Igor

Kruszelnicka, Weronika

Kruszewska, Natalia

Krylov, Alexey

Krylova, Svetlana

Krystkowiak, Michał

Krystosik-Gromadzinska, Agata

Kryzia, Dominik

Krzaczek, Paweł

Kržišnik, Davor

Krzyścin, Janusz
Krzywanski, Jaroslaw

Krzywonos, Małgorzata

Ktena, Aphrodite

$\mathrm{Ku}$, Cheng-Yu

Kuan, Yean-Der

Kubečková, Darja

Kubička, David

Kubik, Andrzej

Kubík, Lubomír

Kubik, Zdenek

Kubota, Keiichi

Kučera, Erik

Kučera, Marián

Kucharčíková, Alžbeta

Kucharska, Karolina

Kuckshinrichs, Wilhelm

Kuczmann, Miklós

Kuczynski, Szymon

Kudasik, Mateusz

Kudelas, Dušan

Kudlek, Edyta

Kudo, Shinji

Kudrna, Petr

Kuester, Matthew

Kühl, Alexander

Kühl, Hans-Detlev

Kühn, Michael

Kuibin, Pavel Anatolievich

Kujawski, Wojciech

Kukacka, Leos

Kukla, Stanisław

Kukulka, David J.

Kulak, Michał

Kularatne, Kanchana

Kulczycka, Joanna

Kulesha, Olga

Kulesz, Barbara

Kulesza, Zbigniew

Kulhandjian, Michel

Kulichikhin, Valery

Kulla, Marián

Kulshreshtha, Prashant

Kumar, Abhash

Kumar, Amit

Kumar, Arkadeep

Kumar, Arun

Kumar, Asheesh

Kumar, Ashish R.

Kumar, Dharmendra

Kumar, Dinesh

Kumar, Harish 
Kumar, Kuldeep

Kumar, Manish

Kumar, Nallapaneni Manoj

Kumar, Navin

Kumar, Nishant

Kumar, Praveen

Kumar, Yedluri Anil

Kumara, Sarath

Kumbár, Vojtěch

Kumtepeli, Volkan

Kunal, Pranaw

Kundracik, Frantisek

Kundu, Arpan

Kunicki, Michał

Kuniewski, Maciej

Kunkel, Julian

Kunugi, Tomoaki

Kunze, Frederik

Kuo, Cheng-Chien

Kuo, Chih-Yu

Kuo, Chung Feng Jeffrey

Kuo, Jian-Long

Kuo, Jim

Kuo, Shu-lung

Kuo, Wen-Ten

Kuo, Yaw-Wen

Kuperman, Alon

Kupiec, Krzysztof

Kupracz, Piotr

Kuravi, Sarada

Kurdyumov, Vadim N.

Kurevija, Tomislav

Kuriqi, Alban

Kurlyandskaya, Galina V.

Kurmaev, Rinat Hanyafievich

Kurnitski, Jarek

Kurokawa, Yasuyoshi

Kurowska, Krystyna

Kurrat, Michael

Kursun, Celal

Kuru, Ergun

Kurushina, Victoria

Kurvinen, Emil

Kurz, Dariusz

Kurzina, Irina A.

Kurzydłowski, Krzysztof J.

Kus, Jolanta

Kůs, Václav

Kusaka, Keisuke

Kušar, Simon

Kusch-Brandt, Sigrid
Kushiro, Noriyuki

Kushkevych, Ivan

Kusnezoff, Mihails

Kustov, Leonid

Kusyk, Janusz

Kutcherov, Vladimir

Kuti, Olawole

Kutija, Martina

Kutsyk, Andriy

Kütt, Lauri

Kuttner, Christian

Kutzner, Rüdiger

Kuusk, Kalle

Kuwahara, Takuya

Kuyukina, Maria S.

Kuzevič, Štefan

Kuzevicova, Zofia

Kuzle, Igor

Kuznetsova, Elizaveta

Kvamme, Bjørn

Kvasha, Andriy

Kwak, Ho Young

Kwak, Keun-Chang

Kwame, Amoah B.O.

Kwapinska, Marzena

Kweon, Hyeongkeun

Kwestarz, Małgorzata

Kwiatkowski, Jerzy

Kwiatkowski, Mirosław

Kwilinski, Aleksy

Kwon, Daeil

Kwon, Hyukjin

Kwon, Jaerock

Kwon, Joseph Sang-Il

Kwon, Jungmin

Kwon, Kye-Si

Kwon, Wookyong

Kyaw, Moe

Kyriakarakos, George

Kyriakopoulos, Grigorios L.

Kyritsis, Evangelos

Kyslan, Karol

Laaksonen, Hannu

Labella, Alessandro

Labus, Aleksandra

Lacey, Gill

Lachmayer, Roland

Laciak, Marek

Łaciak, Mariusz

Lackner, Nina

Lacko, Milan 
Lacko, Roman

Lackowski, Marcin

Ladero, Miguel

Lado, Rita

Ladoux, Philippe

Lafoz, Marcos

Lagasio, Valentina

Lagazzo, Alberto

Lagioia, Giovanni

Łagoda, Tadeusz

Laguë, Claude

Laguna, Oscar H.

Lahiri, Abhishek

Lahtela, Ville

Lai, Bin

Lai, Chi-Ming

Lai, Chun Sing

Lai, Kexing

Lai, Qinghua

Lai, Wei-Chih

Lain, Jenn-Kaie

Lain, Michael

Laín, Santiago

Laine, Markus

Lakatos, Akos

Lakkala, Kaisa

Lakner, Zoltan

Lakshmanan, Padmavathi

Laly, Zacharie

Lam, Eddery

Lam, Garret C. Y.

Lam, Wei Haur

Lamac, Martin

Łamacz, Agata

Lamarche, Louis

Lamas Galdo, María Isabel

Lamb, Jacob J.

Lamberti, Luciano

Lambropoulos, Konstantinos

Lamichhane, Tej

Lamo, Paula

Lampart, Piotr

Lampreia, Suzana

Lampropoulou, Paraskevi

Lan, Dongdong

Lancellotti, Laura

Landa, Imanol

Landi, Carmine

Landi, Daniele

Landi, Gianluca

Landoni, Matteo
Lane-Serff, Gregory

Langar, Sandeep

Lange, Andrzej

Langford, Zach

Langholtz, Matthew

Langlet, Thierry

Lanusse, Patrick

Lanzinha, Joao

Lao, Keng Weng

Laopoulos, Theodore

Łapczyńska-Kordon, Bogusława

Lapenna, Pasquale Eduardo

Łapiński, Marcin

Łapka, Piotr

Lappalainen, Kari

Laprise, Martine

Laptev, Roman

Lara, David

Large, Matthew

Larionov, Vladimir

Larmi, Martti

Larson, David P.

Larsson, Sylvia

Larwa, Barbara

Lascu, Dan Florentin

Lashab, Abderezak

Las-Heras-Casas, Jesús

Lašič Jurković, Damjan

Laskowska, Agnieszka

Lasocki, Jakub

László, Vasa

Latif, Eshrar

Latorrata, Saverio

Latosińska, Jolanta

Latosiński, Paweł

Latré, Steven

Łatuszyńska, Małgorzata

Lau, Anthony

Lau, Stephen Siu-Yu

Laudani, Antonino

Laukkanen, Anssi

Laukkanen, Timo P.

Laura, Romero-Zeron

Lauria, Agostino

Lauria, Davide

Lauria, Stefano

Laurila, Hannu

Laurini, Eleonora

Lavric, Vasile

Law, Cheryl Suwen

Ławińska, Olga 
Lawler, Benjamin

Lawn, Chris

Ławniczak, Paweł

Lawrence, Tom

Lay, Chyi-How

Lazar, Maria

Lazar, Michael

Lazar, Mircea

Lázaro, Jesús

Lazaroiu, Gheorghe

Lazaropoulos, Athanasios G.

Lazarou, Stavros

Lazo, Andrea

Lazopoulos, Konstantinos A.

Lazzaroni, Massimo

Lazzaroni, Roberto

Le Bunetel, Jean-Charles

Le Dréau, Jérôme

Le Gal La Salle, Annie

Le Moyne, Luis

Le Pira, Michela

Le Sache, Estelle

Le Sommer, Nicolas

Le, Tuyen Quang

Le, Viet Hung

Le, Zaiyuan

Leach, Felix C. P.

Leal, Vítor

Leal-Junior, Arnaldo

Lean, Meng $\mathrm{H}$.

Leardini, Fabrice

Leary, Peter

Łebkowski, Andrzej

Lebyodkin, Mikhail A.

Leccadito, Arturo

Leccese, Fabio

Leccese, Francesco

Leccis, Francesca

Leccisi, Enrica

Lechner, Philipp

Lechón, Yolanda

Leckner, Bo

Lederer, Martin

Ledesert, Béatrice

Ledwon, Przemyslaw

Lee, Bok Jik

Lee, Byoung Kuk

Lee, Byung Hee

Lee, Byungjoon

Lee, Chang Jun
Lee, Changhee

Lee, Chengkuo

Lee, Chih-Hao

Lee, Christopher H. T.

Lee, Chuljin

Lee, Chul-Sung

Lee, Chungmok

Lee, Czang-Ho

Lee, Duck-Joo

Lee, Eon Soo

Lee, Eun Yeol

Lee, Gee-Soo (Tongmyong university, Korea)

Lee, Gee-Soo (Youngsan University, Korea)

Lee, Geun Sik

Lee, Gwang Goo

Lee, Hakjin

Lee, Han Eol

Lee, Hansang

Lee, Hee Joon

Lee, Hwansoo

Lee, Hyoung Jin

Lee, Hyoungsoon

Lee, Hyun Woo

Lee, Hyunjin

Lee, Ilkeun

Lee, In Soo

Lee, Jae Suk

Lee, Jae Young

Lee, Jaehan

Lee, Jaesung

Lee, Jaewoo (Chung-Ang University, Korea)

Lee, Jaewoo (Electronics and Telecommun-

ications Research Institute, Korea)

Lee, Jaewook

Lee, James W.

Lee, James Y.

Lee, Jay

Lee, Jechan

Lee, Jee-Keun

Lee, Jeong-Hun

Lee, Johng-Ihl

Lee, Jong-Chul

Lee, Jong-Eun

Lee, Joon Kyu

Lee, Joosung

Lee, Juneseok

Lee, Jung Kyoo

Lee, Junseop

Lee, Kui-Jun

Lee, Kun-Mu

Lee, Kwang Ho 
Lee, Kyo-Beum

Lee, Maw Tien

Lee, Minbaek

Lee, Minhee

Lee, Moonyong

Lee, Mooyeon

Lee, Namkyu

Lee, Paul Tae-Woo

Lee, Pei-Chun

Lee, Ping-Hui

Lee, Pyung Soo

Lee, Ray-Kuang

Lee, Rong-Ho

Lee, Sangho

Lee, Seokhwan

Lee, Seong Hyuk

Lee, Seunghoon

Lee, Seunghwan

Lee, Seung-Hwan

Lee, Seunghyun

Lee, Seungyeo

Lee, Sheng-Long

Lee, Shie-Jue

Lee, Sihyun

Lee, Soo Youn

Lee, Sze Sing

Lee, Tse-Min

Lee, Wen-Jen

Lee, Won-Ju

Lee, Woongkyu

Lee, Yeon-Won

Lee, Yiu Yin Raymond

Lee, Yoo Seok

Lee, Young Duk

Lee, Young II

Lee, Young-Su

Leem, Jung Woo

Leftheriotis, Georgios

Lehikoinen, Antti

Lehmann, Armin

Lehocký, Marián

Lei, Gang

Lei, Xuechen

Leicht, Aleksander

Leigh, Seung-Bok

Leirvik, Thomas

Leitão, Sérgio

Leite, Vicente

Leithardt, Valderi

Leiviskä, Tiina

Lekavičius, Vidas
Leker, Jens

Lekić, Aleksandra

Lekube, Jon

Lekunberri, Edorta Carrascal

Lemanowicz, Marcin

Lembeye, Yves

Lemieux, Patrick

Lemos, José Vieira

Lemu, Hirpa G.

Len, Christophe

Lenhard, Richard

Lens, Hendrik

Lentka, Grzegorz

Lenzi, Ervin K.

Lenzo, Basilio

Léonard, Grégoire

Leonardi, Stefano

Leonforte, Fabrizio

Leong, Ceelia

Leong, Yoong Kit

Leoni, Alfiero

Leonidas, Palilis

Leonori, Stefano

Leonov, Sergey B.

Leonowicz, Zbigniew

Leonzio, Grazia

Leou, Rong-Ceng

Lepadatu, Daniel

Lepoire, David

Leporini, Mariella

Leskarac, Domagoj

Leslie, Lance

Lesnik, Luka

Lester, Henry

Leszczyński, Jacek

Lettieri, Mariateresa

Lettner, Miriam

Leu, Jun-Der

Leukauf, Daniel

Leung, Abraham

Levasseur, Annie

Lévêque, Emmanuel

Levin, Oleg Vladislavovich

Levner, Eugene

Levron, Yoash

Levski, Deyan

Lewandowska, Katarzyna

Lewandowski, Krzysztof

Lewandowski, Mirosław

Lewerenz, Meinert

Lewicki, Arkadiusz 
Lewińska, Paulina

Lewis, Helen

Lewis, Kathy

Lewis, Matthew

Lewis, Randy

Leyland, Pénélope

Lezama, Fernando

Lezsovits, Ferenc

Lezynski, Piotr

Li, Biao

Li, Bing

$\mathrm{Li}, \mathrm{Chao}-\mathrm{Su}$

Li, Chengxi

Li, Chengyu

Li, Chuanyue

Li, Dawei

Li, Dezhi

Li, Duo

Li, Fanghua

Li, Fangxing

Li, Fengwang

Li, Gen

Li, Guiqiang

Li, Hailong

Li, Haiyang

Li, Hang

Li, Hao

$\mathrm{Li}, \mathrm{He}$

Li, Hongru

$\mathrm{Li}, \mathrm{Hu}$

Li, Hua

Li, Huidong

Li, Jia-Han

Li, Jiang

Li, Jianing

Li, Jianlin

Li, Jianmei

$\mathrm{Li}$, Jin

Li, Jinchao

Li, Jinfeng

Li, Jing

Li, Jun

Li, Kaiyuan

Li, Kang

Li, Kun

Li, Lesheng

Li, Liang

Li, Lingwei

Li, Mengying

Li, Mengyu

Li, Min
Li, Ming-Fu

Li, Minghui

Li, Ning

Li, Qibo

Li, Qiusheng

Li, Qiyuan

Li, Rui

Li, Shi Zhong

Li, Simeng

Li, Simon

$\mathrm{Li}$, Sunwei

Li, Tian

Li, Tian-Yi

Li, Weide

Li, Wenjie

Li, Wenliang

Li, Wenming

Li, Wenqin

Li, Wen-Tai

Li, Wenyue

Li, Xiang

Li, Xiao

Li, Xiao Dong

Li, Xiaorong

Li, Xue

Li, Yanfei

Li, Yang

Li, Yangmin

Li, Yanlin

Li, Ying

Li, Yong

Li, Yuanchao

Li, Yun

Li, Zhe

Li, Zheming

Li, Zheng

Li, Zhi

Li, Zhongliang

Li, Zuyi

Lia, Leif

Liang, An

Liang, Chao

Liang, Dawei

Liang, Deqing

Liang, Feng

Liang, Liya

Liang, Wenkai

Liang, Yi

Liang, Youcai

Liang, Zheng

Liang, Zhiming 
Lianos, Panagiotis

Liao, Chien-Sen

Liao, Fanchao

Liao, Hanlin

Liao, Huilian

Liao, Y. Gene

Liberati, Francesco

Libessart, Laurent

Libra, Martin

Libralato, Michele

Lica, Septimiu

Liccardo, Annalisa

Licina, Dusan

Liddle, Brantley T.

Lie, Tek-Tjing

Liem, Peng Hong

Lien, Hsing-Lung

Ligas, Paola

Ligęza, Paweł

Liguori, Simona

Ligus, Grzegorz

Liimatainen, Heikki

Lijewski, Piotr

Likozar, Blaž

Liland, Knut Brede

Lill, Irene

Lillo, Isidoro

Lim, Dae-Kwang

Lim, Dongwon

Lim, Eunjung

Lim, Jae-Han

Lim, Kai Li

Lim, Li Hong Idris

Lim, Ocktaeck

Lim, Teik-Cheng

Lim, Weng Marc

Lim, Young-Il

Lima, Leonardo Dos Santos

Lin, Aqiang

Lin, Bor-Ren

Lin, Chang-Hua

Lin, Chen-Chou

Lin, Cherng-Yuan

Lin, Chih-Hong

Lin, Chun-Hsu

Lin, Chun-Liang

Lin, Deyan

Lin, Jeng-Chyan Muti

Lin, Jia

Lin, Jr-Lung

Lin, Justin Chun Te
Lin, Kuo-Ping

Lin, Lin

Lin, Lingnan

Lin, Linhan

Lin, Shaoyang

Lin, Shih-Mo

Lin, Sijin

Lin, Sin-Jin

Lin, Tyrone $\mathrm{T}$.

Lin, Tzu-En

Lin, Weifeng

Lin, Wenye

Lin, Xianke

Lin, Xiao

Lin, Yu-Chuan

Lin, Yuzhang

Lin, Zhengyu

Lin, Zhibin

Lin, Zhiyuan

Lin, Zhongwei

Linares, José Ignacio

Lind, Pedro G.

Lindberg, Karen Byskov

Lindstad, Elizabeth

Lineykin, Simon

Ling, Florence T.

Ling, Jeen-Min

Ling, Jiazhen

Ling, Steve

Ling, Ziwen

Linguet, Laurent

Lingvay, Iosif

Linkevičius, Edgaras

Linkous, Clovis A.

Linnartz, Jean-Paul

Lio, Wai Hou

Liobikienè, Genovaitè

Lipatnikov, Andrei

Lipczyńska, Aleksandra

Lipecki, Tomasz

Lipian, Michal

Lipiński, Seweryn

Lipovský, Pavol

Liptai, Pavol

Lipuzhin, Ivan

Lis, Andrzej

Lis, Anna

Lis, Robert

Lisa Hsieh, I-Yun

Lisboa, Adriano Chaves

Lisbona, Maria Pilar 
Lisco, Fabiana

Lisetskii, Fedor

Lisi, Luciana

Lisjak, Dragutin

Liska, Adam J.

Liso, Vincenzo

Lisowski, Filip

Liss, Steven N.

Liszkowska, Joanna

Litrán, Salvador Pérez

Litskevich, Dzianis

Litvić, Mladen

Litwin, Wojciech

Liu, Aaron

Liu, Anyao

Liu, Bin (City College of New York, USA)

Liu, Bin (Kansas State University, USA)

Liu, Bin (The University of New South Wales, Australia)

Liu, Bo-Tau

Liu, Chang

Liu, Chuanqi

Liu, Chunhua

Liu, Dong

Liu, Fangda

Liu, Feng

Liu, Fu

Liu, Gang

Liu, Guicheng

Liu, Guigen

Liu, Haidong

Liu, Hao

Liu, Helen

Liu, Hexu

Liu, Hongshun

Liu, Hui

Liu, Huolong

Liu, Jia

Liu, Jiechao

Liu, Jing

Liu, Jui-hung

Liu, Kailong

Liu, Kouqi

Liu, Maning

Liu, Mengmeng

Liu, Qi

Liu, Qili

Liu, Quan

Liu, Ren

Liu, Ren-Shiou
Liu, Rui

Liu, Shyh-Jiun

Liu, Songtao

Liu, Tai-Yi

Liu, Tian-Hua

Liu, Tianyu

Liu, Tzong-shi

Liu, Wan-Yu

Liu, Wei

Liu, Weijia

Liu, Weiping

Liu, Wenzhao

Liu, Xiaobing

Liu, Xiaobo

Liu, Xiao-Hua

Liu, Xiaolei

Liu, Xinyu (Argonne National Laboratory, USA)

Liu, Xinyu (University of Notre Dame, USA)

Liu, Yadong

Liu, Yao-Hsien

Liu, Yaolong

Liu, Yiding

Liu, Yi-Hua

Liu, Ying

Liu, Yingyi

Liu, Yonggang

Liu, Yow-Chyi

Liu, Yuan-Shuai

Liu, Yucheng

Liu, Yujing

Liu, Yunting

Liu, Zhe

Liu, Zongwei

Liudvinavičius, Lionginas

Liutsko, Liudmila

Lizana, Jesus

Llera-Sastresa, Eva

Lloyd, David

Lo Basso, Gianluigi

Lo Frano, Rosa

Lo Monaco, Angela

Lo Schiavo, Alessandro

Lo Storto, Corrado

Lo Vecchio, Carmelo

Lo, Chih-cheng

Lo, Jammie

Lo, Kin Hing

Lobanov, Pavel

Lobato, Killian 
Locmelis, Kristaps

Lodetti, Stefano

Loehle, Craig

Log, Torgrim

Lohr, Christophe

Lo-Iacono-Ferreira, Vanesa G.

Loke, Gabriel

Lokuge, Weena

Lomonaco, Guglielmo

Loncaric, Ivor

Loncarski, Jelena

Long, Linshuang

Long, Nguyen Van Duc

Long, Rui

Longhurst, Philip

Longo, Maria A.

Longo, Sandro

Lonkwic, Paweł

Lónyi, Ferenc

Lopac, Nikola

Lopes, António Manuel Gameiro

Lopes, Fernando

Lopes, Rui Amaral

Lopez Aguirre, Angel Javier

López De Lacalle, Luis Norberto

Lopez Fernandez, Eduardo Jose

López Jiménez, Petra Amparo

López Lambas, María Eugenia

López Martínez, Juan

López Ruiz, Victor R.

López, David Marrero

Lopez, Felix A.

Lopez, Gartzen

López, José María

López, Miguel

López, Montserrat Hernández

Lopez, Omar D.

López, Roberto

Lopez, Santiago M.

Lopez-Chao, Vicente

Lopez-Delgado, Rosendo

López-Estrada, Francisco Ronay

Lopez-Fernandez, Xose

Lopez-Iturri, Peio

López-Jiménez, P. Amparo

López-Linares, Juan Carlos

López-Ochoa, Luis María

López-Ramírez, Simón

Lopez-Santos, Oswaldo

López-Toro, Alberto
López-Villanueva, Juan A.

Lopreite, Milena

Loprencipe, Giuseppe

Lopusniak, Martin

Lordan-Perret, Rebecca

Lorek, Agnieszka

Lorente, Sylvie

Lork, Clement

Löschenbrand, Markus

Losi, Arturo

Lotfian, Saeid

Lou, Fangyuan

Loughney, Sean

Loughrin, John

Loukogeorgaki, Eva

Loures, Luís

Louwen, Atse

Low, Jonathan Sze Choong

Lozac'h, Mickaël

Lozano Guerrero, Antonio José

Lozano-Galant, Jose Antonio

Lozano-Lunar, Angélica

Lozito, Gabriele Maria

Lozynskyy, Andriy

Lu, Bo

$\mathrm{Lu}$, Chao

Lu, Chaoru

Lu, Chia-Liang

Lu, Fei (Drexel University, USA)

Lu, Fei (National Energy Technology

Laboratory, USA)

Lu, Feng

Lu, Hongfang

Lu, Jingyu

Lu, Jingzhi

Lu, Kaiyuan

$\mathrm{Lu}, \mathrm{Li}$ (Singapore)

Lu, Li (USA)

$\mathrm{Lu}$, Meng

$\mathrm{Lu}$, Ming

Lu, Ming-Chang

Lu, Mingyang

Lu, Mingyu

$\mathrm{Lu}$, Shaofeng

$\mathrm{Lu}, \mathrm{Tao}$

Lu, Xuekun

$\mathrm{Lu}, \mathrm{Yi}$

Lu, Yiji

Lu, Yingying

Lu, Yubing

Lu, Zhiyue 
Lubkeman, David

Luboš, Smolík

Luburić, Zora

Lucas, Alexandre

Lucas, Steven

Lucchi, Elena

Lucht, Robert

Lucia, Umberto

Ludlow, David

Ludowicz, Wojciech

Ludwinek, Krzysztof

Lugo, Jesus Eduardo

Luin, Blaž

Luís Marques Macato, André

Luís, Ricardo

Luisetto, Igor

Luiso, Mario

Lujano-Rojas, Juan M.

Lukasik, Rafal

Łukasz Jan, Kapusta

Lukichev, Dmitry V.

Lumentut, Mikail F.

Luna, Diego

Luna, Massimiliano

Lund, Bjørnar

Lungu, Adrian

Luo, Chenglong

Luo, Dan

Luo, Hao

Luo, Hongliang

Luo, Jian

Luo, Maohui

Luo, Win-Jet

Luo, Xiaodong

Luo, Xiaojun

Lupp, Gerd

Lupu, Ciprian

Lupu, Dan

Lupu, Lucian

Luque, Pablo Rodríguez

Luque-Nieto, Miguel Angel

Lurka, Adam

Luszcz, Jaroslaw

Lüth, Stefan

Lutynski, Marcin

Lutzemberger, Giovanni

Lv, Chen

Lv, Haifei

Lygnerud, Kristina

Lykidis, Charalampos

Lynch, Mervyn
Lyon, David

Lyons, Padraig

Lyu, Tao

Lyubenova, Teodora Stoyanova

M'zoughi, Fares

Ma, Chao-Tsung

Ma, Fa-Jun

Ma, Hongjie

Ma, Liuhao

Ma, Naiyang

Ma, Qingqing

$\mathrm{Ma}$, Tingguang

Ma, Wenping

Ma, Xiang

Ma, Xiaoqian

Ma, Yingqun

Ma, Yun

Ma, Yunlong

Ma, Zhenjun

Maag, Alex

Maalouf, Chadi

Maamoun, Ahmed

Máca, Vojtěch

Macaluso, Adriano

Macana Moreno, Carlos Andres

Macas, Martin

Maccarini, Alessandro

MacCarty, Nordica

Maccioni, Marco

Macek, Jan

Macenić, Marija

Macfarlane, Gregor

Machado, Vitor Malo

Machicoane, Nathanael

Macías, Ramón

Maciej, Marek

Maciejewski, Grzegorz

Macioszek, Elżbieta

Maciuk, Kamil

Maciver, Callum

Mackay, Michael

Mackie, Allison

MacPhee, David

Mączka, Wanda

Madadian, Edris

Madaleno, Mara

Madejski, Pawel

Madsen, Dag Øivind

Madzharov, Nikolay

Maeda, Nobuo

Maesano, Mauro 
Mafakheri, Fereshteh

Maffettone, Roberta

Maga, Dušan

Magalhães, Filipe

Magasinski, Alexandre N.

Magazzino, Cosimo

Maggo, Gaetano

Magi, Vinicio

Magliocco, Adriano

Magnani, Natalia

Magnien, Julien

Magnusdottir, Lilja

Magrini, Anna

Magyar, Attila

Mahabadipour, Hamidreza

Mahajan, Devinder

Mahajan, Sagar

Mahajan, Satish M.

Mahan, J. Robert

Mahanti, Aniket

Mahar, Waqas Ahmed

Mahata, Manoj Kumar

Mahato, Neelima

Mahdavi, Mahboobe

Maheshwari, Ramkrishan

Mahmood, Farhan

Mahmoud, Abdelfattah

Mahmoud, Karar

Mahmud, Apel

Mahmud, Khizir

Mahmud, M A Parvez

Mahto, Rakeshkumar

Maia, Thales Alexandre Carvalho

Maigha, Maigha

Mainardis, Matia

Mainka, Julia

Maireles Torres, Pedro Jesus

Maisonneuve, Jonathan

Maiti, Moinak

Maity, Debotyam

Maj, Grzegorz

Majchrzak, Ewa

Majed, Nehreen

Majerus, Steve

Majid, Mehrasa

Majidi Nezhad, Meysam

Majidpour, Mostafa

Majorowicz, Jacek

Majumdar, Amit

Majumdar, Payal

Makaremi, Meysam
Makareviciene, Violeta

Makarov, Dmitriy

Mäkinen, Saku

Makisha, Nikolay

Makoś, Patrycja

Makoschitz, Markus

Makowski, Wacław

Makris, Antonios

Makropoulou, Maria

Makrygiorgou, Despoina I.

Maksimainen, Mikko

Maksyutov, Shamil

Makys, Pavol

Malachowski, Jerzy

Malagon Picon, Beatriz

Malajner, Marko

Malak-Rawlikowska, Agata

Malallah, Ra'ed

Malamaki, Kyriaki-Nefeli D.

Malamakis, Apostolos

Małaszkiewicz, Dorota

Malça, João

Malchik, Fyodor

Maldonado, José-Luis

Male, Sally

Malecha, Ziemowit

Małecki, Krzysztof

Malek Mohammadi, Mohammadreza

Małek, Arkadiusz

Malekian, Reza

Malesza, Wiktor

Maletič, Damjan

Malhotra, Davinder Kumar

Malhotra, Deepika

Malhotra, Mini

Malik, Asif Iqbal

Malik, Om P.

Malik, Sumit

Malinowski, Mateusz

Malinowski, Pawel

Malinowski, Szymon

Malinsky, Jan

Malitckii, Evgenii

Maljkovic, Danica

Malka, Dror

Malkowski, Piotr

Mallik, Awadesh Kumar

Malvić, Tomislav

Malvoni, Maria

Malyuskin, Oleksandr

Mamaev, Ivan Sergeevich 
Mamatov, Aleksandr

Mamkhezri, Jamal

Mammetti, Marco

Mammoli, Andrea Alberto

Mamolos, Andreas

Manai, Bilal

Manamanni, Noureddine

Mañana, Mario

Manandhar, Ashish

Manasis, Christos

Manca, Pier Paolo

Manceau, Rémi

Mancini, Francesco

Mancini, Marco

Mancini, Rock

Mancini, Simone

Manciola, Piergiorgio

Mandal, Mrinmay

Mandelli, Stefano

Manderson, Edward

Mandolini, Marco

Mandrioli, Riccardo

Manetto, Giuseppe Ezio

Manfren, Massimiliano

Manganelli, Benedetto

Manganelli, Matteo

Maniscalco, Rosanna

Manke, David

Mankey, Gary

Mann, Danny D.

Manner, Hans

Mannheim, Viktoria

Manni, Alessandro

Manno, Michele

Mannu, Alberto

Manoj Kumar, Nallapaneni

Manousakis, Nikolaos

Manowska, Anna

Mansinha, Lalu

Mansouri, Abraham

Manuel De Villena, Miguel

Manzano, Osmel

Manzano-Agugliaro, Francisco

Manzo, Maurizio

Manzone, Marco

Mao, Chengsheng

Mao, Xuerui

Maradin, Dario

Marani, Pietro

Maraseni, Tek

Marazuela, Miguel Ángel
Marcello Falcone, Pasquale

Marchelli, Filippo

Marchelli, Maddalena

Marchese, Marco

Marchesoni, Mario

Marchetti, Barbara

Marchi, Beatrice

Marchi, Michela

Marchitto, Luca

Marcin, Wardach

Marco, Bietresato

Marco, Lovera

Marco, Mauri

Marco, Milanese

Marcolini, Alejandro Marano

Marcos-Acevedo, Jorge

Marcoulaki, Effie

Marcu, Ioan Cezar

Marcu, Marius

Marculescu, Cosmin

Marczewska, Magdalena

Mardani, Abbas

Marek, Guzek

Marek, Maciej

Marek-Andrzejewska, Ewelina M.

Marence, Miroslav

Mares, David R.

Maresova, Petra

Marfè, Roberto

Margaritopoulou, Theoni

Margolis, Donald L.

Mari, Carlo

Mari, Federico

Mari, Sonia Irshad

Maria Simona, Raboaca

Maria, Timofeeva

Maria, Vasilyeva

Marian, Dacian-Paul

Mariana, Iorgulescu

Mariani, Antonio

Mariani, Raffaello

Mariano, Silvio

Mariasiu, Florin

Marić, Predrag

Marignetti, Fabrizio

Marinakis, Vangelis

Marinca, Vasile

Marín-Genescà, Marc

Marini, Martino

Marini, Mattia

Marinkovic, Dragan 
Marino, Bruno

Marino, Concettina

Marinoiu, Adriana

Mario, Amelio

Marion, Michaël

Mariscotti, Andrea

Marius, Marcu

Mariyappan, Thambidurai

Marji, Mohammad Fatehi

Mark, Christopher

Markatos, Nikolaos-Christos

Markdahl, Johan

Marken, Frank

Markiewicz, Marietta

Markiewicz, Przemysław

Markou, Giorgos

Marković, Mladen

Markovic, Tomislav

Markowski, Mariusz

Markowski, Piotr Marek

Marks-Bielska, Renata

Marlair, Guy

Mármol, Félix Gómez

Marmolejo-Duarte, Carlos

Marneris, Ilias G.

Marongiu, Andrea

Maroulis, Zacharias

Marques, António

Marques, Bruno

Marques, Isabel Paula

Marques, Luís Miguel

Marquez Alcaide, Abraham

Marquez Ruiz, Alejandro

Márquez, Fausto Pedro García

Marquez, Jaime

Marra, Dario

Marra, Francesco Saverio

Marra, Kristen

Marrasso, Elisa

Marrone, Paola

Marsano, Davide

Marschalko, Marian

Marshall, Ashley R.

Marshall, Paul

Marsset, Bruno

Marszk, Adam

Martegani, Enzo

Martelli, Emanuele

Martens, Betsy

Martí-Ballester, Carmen Pilar

Martí-Herrero, Jaime
Martín De Pozuelo, Ramon

Martín Del Brío, Bonifacio

Martín Martínez, Sergio

Martín, Domingo

Martín, Helena

Martín, Manuel

Martin, Wade E.

Martin-Arnedo, Jacinto

Martinát, Stanislav

Martincic, Emile

Martin-del-Campo, Sergio

Martínez Bohórquez, Miguel Ángel

Martínez García, Miguel

Martínez González, Sergio

Martinez Rojas, Juan Antonio

Martinez, Andrew B.

Martinez, Maria Sofia

Martinez, Sergio

Martinez, Waldyn

Martínez-Espinosa, Rosa María

Martínez-García, Herminio

Martínez-Lillo, José

Martínez-Pagán, Pedro

Martinez-Rodrigo, Fernando

Martinez-Triguero, Joaquin

Martínez-Val, Jose M.

Martín-Garín, Alexander

Martín-Gómez, César

Martini, Alberto

Martín-Martínez, Francisco J.

Martino, Luca

Martino, Marco

Martino, Michele

Martinopoulos, Georgios

Martinovic, Zeljko

Martín-Ramos, Juan A.

Martins Afonso, Marco

Martins Gomes, João Manuel

Martins, Ana Lucia

Martins, António A.

Martins, António Gomes

Martins, António Pina

Martins, Fernando Ramos

Martins, Gilberto

Martins, João

Martins, Jorge

Martins, Luísa Margarida

Martins, Nelson

Martirano, Luigi

Martišauskas, Linas

Marton, Isabel 
Martone, Ivo

Marucci, Alvaro

Marugán-Cruz, Carolina

Maruşić, Ante

Marx, Klemens

Marzband, Mousa

Marzeddu, Simone

Marzinotto, Massimo

Mas'ud, Abdullahi Abubakar

Máša, Vítězslav

Masamune, Sadao

Masaryk, Michal

Masayuki, Anyoji

Masdías-Bonome, Antonio

Masek, Pavel

Maset, Enrique

Mashima, Daisuke

Masini, Barbara Mavì

Maskeliunas, Rytis

Maskell, Daniel

Maslak, Mariusz

Mašláni, Alan

Maslov, Andrey V.

Masnicki, Romuald

Masood, Nahid Al

Masoudi Soltani, Salman

Masoudi, Ali

Maspero, Federico

Masselli, Claudia

Massidda, Luca

Massier, Tobias

Mastellone, Maria Laura

Masterson, Douglas

Mastino, Costantino Carlo

Mastny, Petr

Mastrocinque, Ernesto

Mastromauro, Rosa Anna

Mastropasqua, Luca

Masuhara, Naoki

Masum, Shakil

Masurkar, Nirul

Masuta, Taisuke

Mata, Maria Del Carmen

Mata-Lima, Herlander

Matas, José

Matas, Richard

Matasaru, Petre Daniel

Matassa, Silvio
Mateo, Carlos M.

Mateo-Garcia, Monica

Mateo-Lázaro, Jesús

Materazzi, Massimiliano

Mateus, Dina M. R.

Mateus, Ricardo

Matharage, Shanika

Mathelin, Lionel

Mather, Glenn

Mathew, Kattathu

Mathew, Paul

Mathies, Florian

Mathieu, Olivier

Mathieu, Sébastien

Matijević, Mario

Matijošius, Jonas

Matko, Vojko

Matos, Joao Nuno

Matousek, Roman

Matricciani, Emilio

Matsika, Emmanuel

Matsoukas, Themis

Matsumoto, Hiroshi

Matsumoto, Ken'ichi

Matsumoto, Satoshi

Matsuo, Tomohito

Matsushima, Yuta

Matsuura, Daisuke

Mattarocci, Gianluca

Matteo, Edward N.

Matteo, Laura

Mattias, Gaglio

Mattiazzo, Giuliana

Mattingly, Stephen P.

Matúš, Miloš

Matušů, Radek

Matuszek, Karolina

Matykiewicz, Danuta

Mauleón, Ignacio

Mauri, Luca

Mauri, Roberto

Mauricio, Juan Manuel

Mauriello, Filomena

Mauriello, Francesco

Mauro, Alessandro

Mauro, Gerardo Maria

Mauro, Giovanni

Mavromatakis, Fotis

Matavalam, Amarsagar Reddy Ramapuram Mavromatidis, Lazaros

Mateescu, Carmen

Mateev, Valentin

Mavropoulos, Georgios

Maximov, Maxim Yu 
Maxineasa, Sebastian George

Maxwell, Radin

May, Nils

May, Roland

Mayer, Daniel

Mayet, Clément

Mazadiego, Luis F.

Mazancová, Jana

Mazarakos, Thomas P.

Mažeikienè, Aušra

Mazela, Bartłomiej

Mazhar, Ilyas

Mazierski, Paweł

Mazinani, Saeed

Mazon, Jordi

Mazurek, Paweł

Mazurkiewicz, Jakub

Mazza, Daniela

Mazzanti, Giovanni

Mazzei, Lorenzo

Mazzeo, Domenico

Mazzoleni, Claudio

Mba-Wright, Mark

McCann, Roy

McClure, Mark

McConkie, Eileen

McDanel, Bradley

McDaniel, Patrick

McDermott, Thomas

McDonald, Robbie

McDonnell, Kevin

McEldowney, John

McFarlane, Joanna

McGrattan, Kevin B.

McIlvenny, Jason

McIntyre, Michael

McKinley, Allan

McKinley, Arnold

Mckitrick, Ross

McLellan, Ben

McLellan, Iain

McMaster, Scott A.

McNaughton, James

McNeill, Neville

Meara, Paul

Mecca, Umberto

Mečiarová, Ludmila

Meda-Campaña, Jesús Alberto

Medica-Viola, Vedran

Medici, Vasco

Medina Carrasco, Santiago
Medina Quero, Javier

Medina Quesada, Ángeles

Medina, Agustin Valera

Medrano, Marc

Medved', Dušan

Medved, Tomi

Medvedev, Dmitry

Medyna, Galyna

Medyńska-Juraszek, Agnieszka

Meegahapola, Lasantha

Meegoda, Jay N.

Meek, Sanford

Meeker, David

Meen, Teen-Hang

Meena, Nand K.

Meesenburg, Wiebke

Megía, Pedro J.

Megyesiová, Silvia

Mehdipour, Iman

Mehmood, Faizan

Mehmood, Khawaja Khalid

Mehmood, Rashid

Mehrali, Mohammad

Mehran, Kamyar

Mehrandezh, Mehran

Mehrtash, Mahdi

Mehta, Mohit

Mei, Jun

Mei, Yunhui

Meidute-Kavaliauskiene, Ieva

Meier, Alexandra Von

Mekhilef, Saad

Melaika, Mindaugas

Mélard, Guy

Melatti, Igor

Melchiorre, Erik

Meletlidou, Efi

Melgar, Sergio Gómez

Melgoza-Vázquez, Enrique

Melka, Bartlomiej

Mellal, Mohamed Arezki

Meloni, Carlo

Meloni, Eugenio

Memik, Seda Ogrenci

Memmolo, Vittorio

Memmott, Matthew

Men, Zhongxian

Menaa, Farid

Mendes, Adélio

Mendes, André

Mendes, Mário C. 
Mendes, Paulo

Méndez, Juan A.

Méndez-Suárez, Mariano

Mendizabal, Jaizki

Mendizábal, Rafael

Mendoza, Daniel L.

Mendrinos, Dimitrios

Mendyk, Aleksander

Menegaki, Angeliki

Meneghetti, Giovanni

Menendez, Javier

Menéndez, Miguel

Meneses, Lisandra

Meng, Fanlin

Meng, Ke

Meng, Meng

Meng, Weizhi

Meng, Zhaozong

Menichetti, Marco

Mennerich, Artur

Menoufi, Karim

Mentel, Grzegorz

Mera, José Manuel

Mercader-Moyano, Pilar

Merchan-Merchan, Wilson

Merchan-Villalba, Luis Ramon

Mercorelli, Paolo

Mereuță, Elena

Mériguet, Guillaume

Merino-Garcia, Ivan

Merk, Bruno

Merkisz-Guranowska, Agnieszka

Merlo, Marco

Mernik, Marjan

Merrett, Craig G.

Merz, Rainer

Meschede, Henning

Mesquita, Luis

Messina, Giuseppe

Messner, Mark C.

Mestas-Nuñez, Alberto M.

Mester, Christian

Mestriner, Daniele

Mets, Tõnis

Meyal, Alireza

Meyer, Ina

Meyer, Richard T.

Meyer-Kohlstock, Daniel

Mezei, József

Meziti, Alexandra

Miah, M. Shamim
Miah, Mohammad Islam

Miaja, Pablo F.

Miao, Yu

Miatto, Alessio

Micallef, Daniel

Micangeli, Andrea

Miccio, Francesco

Michael, Bryane

Michailides, Constantine

Michalak, Aneta

Michalak, Jarosław

Michaud, Morgane

Michel, Frederick

Michel, Jean-Bernard

Michelassi, Vittorio

Micheli, Leonardo

Micheli, Silvia

Michna, Michał

Michopoulos, Apostolos

Miciuła, Ireneusz

Micu, Dan Doru

Mieczkowski, Grzegorz

Miecznik, Maciej

Mieg, Harald

Miele, Ermanno

Mielewczyk-Gryn, Aleksandra

Miguel, Antonio

Miguel, Silvia

Mihaescu, Lucian

Mihai, Costica

Mihai, Cruceru

Mihaiescu, Tania Cecilia

Mihailov, Nikolay

Mihalache, Sanda Florentina

Mihalic, Rafael

Mihelič, Rok

Mihet-Popa, Lucian

Mijo, Nikolic

Mikalauskienè, Asta

Mikayilov, Jeyhun I.

Mikhailova, Daria

Mikhaylov, Alexey

Mikielewicz, Dariusz

Miklos, Zoltan

Miklosevic, Kresimir

Mikolajczyk, Tadeusz

Mikropoulos, Pantelis N.

Mikulić, Davor

Mikulik, Jerzy

Mikulski, Dawid

Milad, Benmadi 
Milanés Montero, María Isabel

Milani, Dia

Milani, Massimo

Milano, Federico

Milanovic, Milos

Milazzo, Adriano

Milazzo, Maria Francesca

Milčius, Darius

Milciuviene, Saule

Milczarek, Adam

Milecki, Andrzej

Miled, Zina Ben

Milewski, Jaroslaw

Milicevic, Kruno

Milichovský, František

Miliozzi, Adio

Milis, Kevin

Miliucci, Marco

Miljavec, Damijan

Millar, Michael-Allan

Miller, Boris

Miller, Gregory

Miller, Jimmie

Miller, Lindsay

Miller, Piotr

Miller, Reinhard

Millithaler, Jean-François

Milojević-Rakić, Maja

Milovanović, Bojan

Min, Baehyun

Min, Changgi

Min, Daiki

Min, Jihoon

Minak, Giangiacomo

Minakshi, Manickam

Minami, Ichiro

Minciuc, Eduard

Mindemark, Jonas

Minea, Alina Adriana

Minea, Marius

Mingant, Remy

Mingesz, Robert

Mingotti, Alessandro

Minnaert, Ben

Minussi, Carlos Roberto

Minuto, Francesco Demetrio

Miranda Barbosa, Edesio

Miranda, João Mário

Miranda, María Teresa

Mirante, Fátima I. C.

Mirás-Avalos, José Manuel
Mirauda, Domenica

Mirbozorgi, S. Abdollah

Mirea, Radu

Mirek, Paweł

Miró, Guillermo

Miron, Dumitru

Mironova, Maria

Misa, Rafał

Mischie, Septimiu

Mishra, Aashwin

Mishra, Asitav

Mishra, Kuber

Mishra, Partha

Mishra, Sambeet

Mišík, Matúš

Misirlis, Dimitrios

Misiunaite, Ieva

Miska, Stefan Z.

Miśkiewicz, Radosław

Mistewicz, Krystian

Mistrick, Richard G.

Mišurec, Jiř́i

Misyura, Sergey Y.

Mitoraj, Mariusz

Mitoulis, Stergios

Mitran, Raul-Augustin

Mitronikas, Epaminondas

Mitrović, Nebojša

Mittal, Anuj

Mittal, Nitesh

Mittelbach, Martin

Mityakov, Andrey V.

Miura, Yushi

Miyafuji, Hisashi

Miyazaki, Koji

Mizielińska, Małgorzata

Mizik, Tamás

Mizsei, János

Mizsey, Peter

Mizutani, Norimi

Mizzi, Luke

Mjahed, Mostafa

Mladineo, Marko

Mlejnek, Pavel

Mlonka-Mędrala, Agata

Mlot, Adrian

Młynarczyk, Przemysław

Moazami, Amin

Moazeni, Faegheh

Mobedi, Moghtada

Mobilia, Mirka 
Mocanu, Mariana

Mocera, Francesco

Mochamad, Rian Fatah

Mochiyama, Shiu

Mochizuki, Hiroyasu

Modarresi, Mohammad Sadegh

Modrak, Vladimir

Moeini, Rana

Moestedt, Jan

Mofakham, Amir A.

Moggi, Sara

Moghadam, Amir Ali Amiri

Moghaddam, Hesam S.

Moghaddam, Hussein

Moghimi, M. A.

Mohabeer, Chetna

Mohaghegh, Zahra

Mohamad-Hussein, Assef

Mohamed, Bassam

Mohamed, Elamin

Mohamed, Ibrahim

Mohamed, Moataz

Mohamed-Seghir, Mostefa

Mohammad, Mostak

Mohammadi, Ali

Mohammadi, Alireza

Mohammadi, Babak

Mohammadi, Fazel

Mohammadi, Mohammad Ebrahim

Mohammadi, Mohammad Hossain

Mohammadi, Sajjad

Mohammadi, Saleh

Mohammadian, Hamid Doost

Mohammadian, Majid

Mohammadian, Shahabeddin K.

Mohammed, Abdul Samad

Mohammed, Hussein A.

Mohammed, Iman

Mohammed, Nabil

Mohan, Gowtham

Mohanty, Bibhu

Mohareb, Eugene

Mohd Alsofyani, Ibrahim

Mohelnikova, Jitka

Mohit, Mittal

Mohlakoana, Nthabiseng

Mohles, Volker

Mohr, Steve

Moiceanu, Georgiana

Moineau-Chane-Ching, Kathleen I.

Moioli, Stefania
Moiseev, Nikita

Moita, Ana

Moiz, Ahmed Abdul

Moiz, Syed Abdul

Mojiri, Ahmad

Mojiri, Amin

Mokhtari, Hossein

Mokhtari, Mehdi

Moldovan, Macedon

Moldovanu, Dan

Moles, Richard

Molina-García, Ángel

Molina-Moreno, Valentín

Molinari, Nicola

Molina-Solana, Miguel

Moliner, Cristina

Molines Llodrá, Jorge

Molla, Md. Ashraful Islam

Möllerström, Erik

Molnar, Oleksandr

Molnar, Peter

Molnar-Matei, Florin

Molodtsova, Tanya

Molognoni, Daniele

Momchilov, Anton

Momete, Daniela

Mon, Yi-Jen

Monai, Matteo

Moncecchi, Matteo

Monclús, Hèctor

Mondal, Abrez

Mondragón, Rosa

Monedero, Esperanza

Monfared, Radmehr P.

Monforti Ferrario, Andrea

Monforti-Ferrario, Fabio

Mongiardo, Mauro

Monica, Costea

Monieta, Jan

Moñino, Antonio

Monjo, Lluis

Montambault, Serge

Montana, Francesco

Montanari, Gian Carlo

Monteil-Rivera, Fanny

Monteiro Da Silva, Sandra

Monteiro, Eliseu

Monteiro, Vítor

Montel, Francois

Montenegro, Davis

Montenegro, Sergio 
Montero-Chacón, Francisco

Montisci, Augusto

Montllor, Joan

Montoya, Alejandro

Montoya, Oscar Danilo

Montuori, Lina

Monzón Chavarrías, Marta

Monzon Verona, Jose Miguel

Moon, Chanwoo

Moon, Hokyu

Moore, Keegan

Moore, Wayne

Moosmuller, Hans

Moradikazerouni, Alireza

Moradisizkoohi, Hadi

Morag, Yahav

Mora-Gimeno, Francisco Jose

Moral-Carcedo, Julián

Morales de la Rosa, Silvia

Morales-Conde, Maria Jesús

Morán Álvarez, Antonio

Moravek, Jan

Morchenko, Alexander

Morea, Donato

Moreau, Stéphane

Morel, Hervé

Morency, François

Moreno García, Jaime

Moreno, Andrés

Moreno, Antonio D.

Moreno, Efrain Carpintero

Moreno, Maria Angeles

Moreno-Eguilaz, Juan Manuel

Moreno-Garcia, Isabel María

Moreno-García, Manuel

Moreno-Guerrero, Antonio-José

Moreno-Munoz, Antonio

Moretti, Sacha

Moretto, Michele

Morgan, Kevin

Morganti, Fabrice

Mori, Kotaro

Mori, Taro

Moriarty, Patrick

Morinigo-Sotelo, Daniel

Morkūnas, Mangirdas

Morkvenaite-Vilkonciene, Inga

Morley, Bruce

Morlier, Arnaud

Moro Visconti, Roberto

Moro, Federico
Morone, Piergiuseppe

Moroni, Davide

Moroni, Stefano

Morosuk, Tatiana

Morovatiyan, Mohammadrasool

Morozyuk, Tetyana

Morris, Nicholas John

Morrone, Biagio

Morrone, Pietropaolo

Morsalin, Sayidul

Morshed, Mohammad Javad

Mosa, Islam

Mosaad, Mohamed I.

Mosavi, Amir

Moscardini, Marigrazia

Moscariello, Mariagiovanna

Mösch, Mario

Moschoyiannis, S.

Moser, David

Moses, Paul

Moshou, Alexandra

Mosiałek, Michał

Moskalik, Tadeusz

Moslem, Ali Ibrahim

Moss, Steve

Mostegl, Nina M.

Mota-Babiloni, Adrián

Motoyama, Hideki

Motra, Hem Bahadur

Mottola, Fabio

Motuzienè, Violeta

Moujaees, Samir

Mouli-Castillo, Julien

Moulogianni, Christina

Moulos, Vrettos

Moura Paredes, Guilherme

Moury, Sanjida

Mousa, Elsayed

Mousas, Christos

Moussaoui, Younes

Moustakas, Konstantinos

Mowry, Greg

Moya, Antonio A.

Mozaffari, M.Hamed

Możaryn, Jakub

Mozayeni, Hamidreza

Mozdzierz, Marcin

Mozhzhukhina, Nataliia

Mpanda Mabwe, Augustin

Mračková, Eva

Mroczkowska-Szerszeń, Maja 
Mróz, Tomasz

Mrzljak, Vedran

Muc, Adam

Mucchi, Emiliano

Mucha, Maria

Muche, Gerhard

Mucsi, Zoltán

Mude, Kishore Naik

Mudryk, Krzysztof

Muftahov, Ildar

Muhammad Ali, Hafiz

Muhammad-Sukki, Firdaus

Muhr, Michael

Mukherjee, Amlan

Mukherjee, Nilanjan

Mukherjee, Sankha

Mukherjee, Santanu

Mukherjee, Soumya

Mukhopadhyay, Shayok

Mulchandani, Anjali

Muljadi, Bagus Putra

Mullen, Charles

Müller, Gerhard

Müller, Julian Marius

Müller, Karsten

Mullins, Oliver C.

Multanen, Victor

Mulville, Mark

Mun, Junyoung

Muncharaz, Javier Oliver

Muneer, Tariq

Muniak, Damian Piotr

Munir, Tariq M.

Muñoz Domínguez, Marta

Muñoz, Delfina

Munoz, Fabio

Muñoz-García, Miguel A.

Muñoz-González, Carmen Ma

Muñoz-Iglesias, Victoria

Munshi, Talat

Muntean, Nicolae

Muntean, Sebastian

Munteanu, Calin

Muntendam-Bos, Annemarie

Muradin, Magdalena

Murai, Motohiko

Murai, Yuichi

Muráth, Szabolcs

Murawski, Lech

Murcia Leon, Juan Pablo

Muresan, Andruta
Muresan, Cristina

Muresan, Iulia

Muresan, Vlad

Murinova, Petra

Murkovic, Michael

Muro Jr, Aldo

Murphy, Michael D.

Murray, Alexander

Murray, Renique J.

Murshed, Syed Monjur

Murshid, Nimer

Murtagh, Fionn

Murthy, Tammana S. R. C.

Murzin, Dmitry

Musca, Rossano

Muscio, Alberto

Musiał, Michał

Musilek, Petr

Musmarra, Dino

Musolino, Antonino

Musselwhite, Charles

Mustafa, Ahmed

Muster, Tim

Musto, Marilena

Musuc, Adina Magdalena

Musumeci, Salvatore

Musznicki, Piotr

Musz-Pomorska, Anna

Musztyfaga-Staszuk, Małgorzata

Muszyński, Tomasz

Mutascu, Mihai

Muto, Masaya

Muttin, Frédéric

Mwesigye, Aggrey

Myeong, Seunghwan

Myers, Mike

Myers, T. G.

Mynett, Arthur

Myöhänen, Kari

Mystkowski, Arkadiusz

Myung, Jaewook

$\mathrm{Na}$, Kyungmin

$\mathrm{Na}$, Kyungsu

$\mathrm{Na}$, Seunguk

$\mathrm{Na}$, Woonki

Nabavi, Seyedfakhreddin

Nabi, Saleh

Nadai, Chiara

Nadais, Helena

Naderi, Ehsan

Naderian, Ali 
Nadolny, Zbigniew

Naeimipour, Ali

Naeini, Mahshid R.

Nafkha, Rafik

Nag, Soumyadeep

Nagai, Keiji

Nagaj, Rafal

Nagane, Satyawan

Nagarajan, Sanjay

Nagasawa, Keisuke

Nagata, Harunori

Nagi, Łukasz

Nagisetty, Raja

Nagpal, Himanshu

Naguib, Michael

Nagy, Stanislaw

Nah, Wansoo

Naik Parrikar, Prathmesh

Naik, Sambit Prasanajit

Nainar, Karthikeyan

Nair, Arun Sukumaran

Nair, Rawindaran

Najafi, Foad

Najser, Jan

Nakajima, Tadahiro

Nakamura, Takashi

Nakano, Katsuyuki

Nakarmi, Upama

Nakashima, Naotoshi

Nakashima, Takuji

Nakata, Shunji

Nakata, Toshihiko

Nakata, Yoshihiro

Nakayama, Akira

Nakayasu, Yuta

Nakutis, Žilvinas

Naldi, Claudia

Nam, Bo

Nam, Hyungseok

Nam, Inho

Nam, Seong-Nam

Nam, Soon-Ryul

Nam, Yunyoung

Nami, Hossein

Namysłowska-Wilczyńska, Barbara

Nan, Fany

Nanaki, Evanthia

Nanami, Norimichi

Nandi, Tarak Nath

Nanjundaswamy, Ananda

Nápek, Jaroslav
Napierała, Michał

Napieralska-Juszczak, Ewa

Napolitano, Fabio

Naraghi, Mohammad

Narasingam, Abhinav

Narayan, Nishant

Narayan, Seema

Narciso, João

Nardino, Marianna

Narimani, Mohammad Rasoul

Narita, Takayoshi

Narkuniene, Asta

Narula, Kapil

Naščáková, Jana

Nascimento, Luís Adriano Santos Do

Naser, M. Z.

Naseri, Masoud

Nasidi, Muhammad

Nasir, Mashood

Nasiri, Fuzhan

Nassif, Nabil

Nastac, Silviu

Nastasi, Benedetto

Nastasi, Nicola

Natili, Francesco

Natsui, Shungo

Nautiyal, Amit

Navarra, Alessandro

Navarro, Francisco Javier

Navarro-Camba, Enrique A.

Navau, Carles

Navin, Chelliah V.

Năvrăpescu, Valentin

Nawalany, Grzegorz

Nawrot, Tadeusz

Nayeri, Christian

Nazari, Behzad

Nazari, Shima

Nazari-Heris, Morteza

Nazemi, Mohammadreza

Nazir, M. Tariq

Nchor, Dennis

Ndawula, Mike Brian

Ndibanje, Bruce

Nduka, Onyema S.

Neag, Emilia

Neagoe, Mircea

Neagu, Bogdan Constantin

Neagu, Olimpia

Neamt, Liviu

Nebot-Andrés, Laura 
Nechaev, Victor

Nechifor, Gheorghe

Nęcka, Krzysztof

Necula, Horia

Nedelcu, Dorian

Nedelcu, Dumitru

Neetzow, Paul

Negahdar, Leila

Negishi, Shintaro

Negreanu, Gabriel

Negrello, Francesca

Negro, Enrico

Nehler, Therese

Neighbour, Gareth

Nejad, Ali Farokhi

Nelson, Brad

Nelson, Tim

Nemchinova, Nina

Nemec, Mitja

Nemer, Maysaa

Nemes, Ciprian

Németh, Áron

Nemnes, George Alexandru

Nemoianu, Iosif Vasile

Nemova, Darya

Nemś, Artur

Nemś, Magdalena

Nenadic, Nenad

Nenning, Andreas

Neophytou, Neophytos

Nepal, Rabindra

Neri, Igor

Neri, Manuela

Nermend, Kesra

Neshat, Mehdi

Nespoli, Alfredo

Nesticò, Antonio

Nestle, Nikolaus

Netskina, Olga

Neubauer, Jeremy

Neugebauer, Maciej

Neumann, Tomasz

Neunaber, Ingrid

Neustupa, Jiří

Nevalainen, Paavo

Neves Lousada, Sérgio António

Neves, Luís Pires

Nevidomskaya, Dina G.

Newbery, David

Newman, Steven E.

Neyer, Daniel
Neyestani, Nilufar

Ng'ombe, John N.

Nganguia, Herve

Ngo, Ha-Duong

Ngo, Son I.

Ngoc, Tran Huynh

Ngoc-Phi, Nguyen

Nguyen Hong, Nhung

Nguyen, Bao-Huy

Nguyen, Dang Mao

Nguyen, Dinh Tung

Nguyen, Dung Anh

Nguyen, Duy-Dinh

Nguyen, Gia Minh Thao

Nguyen, Giang

Nguyen, Ha Thi

Nguyen, Huong Thu

Nguyen, Khac Long

Nguyen, Khoa

Nguyen, Minh Tuan

Nguyen, Minh-Khai

Nguyen, Nga

Nguyen, Quang D.

Nguyen, Thien D.

Nguyen, Tien Thang

Nguyen, Toan

Nguyen, Tung-Lam

Nguyen, Van Khanh

Nguyen, Van-Dang

Nguyen, Van-Ha

Nguyen, Xuan Bac

Nguyen-Van, Triet

Nhuchhen, Daya

$\mathrm{Ni}$, Jiangfeng

Niayesh, Kaveh

Niaz, Muhammad Tabish

Niazmand, Amirreza

Niccolai, Alessandro

Nicholls, David L.

Nicholson, Schuyler

Nick, Hamid

Nick, Hamidreza M.

Nicola, Lolli

Nicolae, Badea

Nicolae, Cordos

Nicolae, Ileana Diana

Nicolas, Andrea

Nicolini, Andrea

Niculescu, Rodica

$\mathrm{Nie}$, Mengyan

Nie, Zhenggang 
Niedbalski, Zbigniew

Niedoba, Tomasz

Niedzwiecki, Lukasz

Niegodajew, Paweł

Niehoff, Philip

Nielsen, Henrik Kofoed

Nielsen, Kaspar Kirstein

Nielsen, Søren Nors

Nielsen, Toke Rammer

Niemann, Jorg

Nieminen, Kaarlo

Nieße, Astrid

Nieto, Ignacio Martín

Niewelt, Tim

Niewiadomski, Adam

Niewiara, Lukasz

Niezgoda-Zelazko, Beata

Niguchi, Noboru

Nikas, Alexandros

Nikitenko, Anatolii

Nikitenko, Sergey I.

Nikodinoska, Dragana

Nikolaev, Petr

Nikolaidis, Theoklis

Nikolakopoulos, Pantelis G.

Nikolaos, Apostolopoulos

Nikolaos, Nikolopoulos

Nikolay Ivanovich, Vatin

Nikolic, Danijela

Nikolic, Jelena

Nikolic, Mijo

Nikolic, Ruzica

Nikologianni, Anastasia

Nikolopoulos, Christos

Nikolopoulou, Kleopatra

Nikolovski, Srete

Nikończuk, Piotr

Nikoueeyan, Pourya

Nikovski, Daniel

Nikulin, Artem

Nilsson, Joakim

Nimmagadda, Shastri

Ning, Dezhi

Ning, Jinqiang

Ning, Puqi

Ninikas, Konstantinos

Niri, Mona Faraji

Nishi, Yoshiki

Nishioka, Shuichiro

Nishiuchi, Hiroaki

Nishiwaki, Tomoya
Nisticò, Roberto

Nita, Irina

Niu, Yuling

Niv, Avi

Nivedita, Nivedita

Nivin, Valentin A.

Nizami, Abdul-Sattar

Nizetic, Sandro

Njuguna, James

Nnaemeka Vincent, Emodi

No, Sang-Tae

Noack, Bernd R.

Noble, Donald

Nocente, Alessandro

Nocera, Francesco

Nocera, Silvio

Nocivelli, Lorenzo

Nocoń, Adrian

Noei, Heshmat

Noetinger, Benoît

Noga, Marcin

Nogueira, Teresa

Noh, Yeon Sik

Nole, Michael

Nolte, Christopher G.

Nolting, Lars

Noma, Naoki

Nooraiepour, Mohammad

Noor-A-Rahim, Md

Norbisrath, Jan Henrik

Nord, Natasa

Nordtveit, Ernst

Norek, Małgorzata

Noreus, Dag

Nørgaard, Per

Noro, Marco

Norrlöf, Mikael

Northrop, Paul

Norwood, Zack

Nosek, Radovan

Noszczyńska, Magdalena

Notaro, Sandra

Notton, Gilles

Nour, Mutasim

Nourian, Amir

Nourozieh, Hossein

Noussan, Michel

Novak, Jiri

Novak, Martin

Novak, Miroslav

Novak-Mavar, Karolina 
Novakovic, Vojislav

Novo, Luís

Novotny, Pavel

Nowaczyk, Sławomir

Nowak, Andrzej

Nowak, Grzegorz

Nowak, Krzysztof

Nowak, Sascha

Nowak-Woźny, Dorota

Nowicki, Michał

Nowogonska, Beata

Nowrot, Andrzej

Nozariasbmarz, Amin

Nozzoli, Francesco

Ntaikou, Ioanna

Ntanos, Stamatios

Ntougias, Spyridon

Ntzeremes, Panagiotis

Nuchkrua, Thanana

Nuez, Ignacio

Nunes, Leonel Jorge Ribeiro

Núñez, Alfredo

Núñez, Luis Ramón

Nuñez, Neftali

Nunna, Bharath Babu

Nunnari, Giuseppe

Nutaro, James

Nutskova, Mariia V.

Nuzzo, Stefano

Nwajana, Augustine O.

Nyaaba, Wedam

Nyangon, Joseph

Nyantekyi-Kwakye, Baafour

Nygaard, Runar

Nygård, Heidi

Nyiwul, Linus Mabughi

Nzila, Alexis

O'Brien, Paul

O’Byrne, Michael

O'Donnell, Terence

O'Hern, Steve

O'Neill, Robert

O'Sullivan, Paul

Oancea, Florin

Obafemi, A. P. Olukoya

Obaidat, Muath A.

Obeng-Odoom, Franklin

Oberegger, Ulrich Filippi

Obidowski, Damian

Obidziński, Sławomir

Obracaj, Dariusz
Obrecht, Matevz

Obstawski, Paweł

Obushevs, Artjoms

Ochkov, Valeriy

Ochoa, Danny

Ochodek, Tadeáš

Ochowiak, Marek

Octrue, Michel

Oda, Takuya

Oda, Yutaka

Odagiu, Antonia

Odeh, Saad

Odriozola, José

Oehlschlaeger, Matt

Oettingen, Mikolaj

Oezkaya, Ekrem

Ofman, Piotr

Ogasawara, Hirohito

Oggeri, Claudio

Oginni, Oluwatosin

Ogino, Akifumi

Ogórek, Rafał

Oguntimein, Gbekeloluwa B.

Ogura, Daisuke

Ogut, Mehmet

Oh, Eunsung

Oh, HyungSeon

Oh, Inha

Oh, Jaewon

Oh, Ki Yong

$\mathrm{OH}$, Kwangchul

Oh, Min-wook

Oh, Sukjoon

Oh, Taekeun

Ohadi, Michael

Ohgaki, Hideaki

Ohlhorst, Doerte

Ohodnicki, Paul

Ohtake, Hideaki

Oikonomopoulos, Ioannis

Oikonomou, Panagiotis

Ojo, Peter

Ojovan, Michael I.

Okamoto, Satoru

Okamoto, Tatsuki

Okhay, Olena I.

Oko, Eni

Okolo, Patrick Nwosa

Okonkwo, Christopher

Okoro, Oseweuba

Oksa, Maria 
Oku, Takeo

Okubo, Kazuaki

Okumiya, Masaya

Okumura, Susumu

Okyay, Gizem

Olabi, Abdul-Ghani

Oladosu, Gbadebo

Olama, Mohammed

Olczak, Piotr

Olczykowski, Zbigniew

Oleinik, Phelype Haron

Olejnik, Bartosz

Oleka, Emmanuel

Oleksandrovych, Illiashenko Oleg

Oleksiak, Ariel

Oleksik, Mihaela

Olesen, Bjarne

Oliveira Panão, Miguel R.

Oliveira Pinto, José Gabriel

Oliveira, Fabricio

Oliver, Matthew

Oliverio, Manuela

Olivier, Jean-christophe

Olivieri, Carlo

Olivieri, Francesca

Olivieri, Lorenzo

Olmedillas Blanco, Blanca

Olmos, Luis

Olondriz, Joannes

Olovsson, Conny

Olšiak, Róbert

Olsson, Linda

Olszewski, Robert

Oltean, Gabriel

Olteanu, Severus

Oluleye, Gbemi

Olvera Trejo, Daniel

Omar, Faye

Omell, Benjamin

Omer, Joshua

Omitaomu, Olufemi A.

Omrani, Sara

Omrany, Hossein

Onat, Altan

Oncioiu, Ionica

Onea, Alexandru

Oñederra, Oier

Ong, Hwai Chyuan

Onica, Ilie

Onireti, Oluwakayode

Onishi, Viviani
Onori, Simona

Onwudili, Jude

Opala, Michal

Opdenakker, Raymond J.G.

Operat, Agnieszka

Opila, Daniel

Opila, Robert

Opra, Denis

Oprea, Claudiu Alexandru

Oprea, Corneliu Ioan

Oprea, Simona

Oprescu, Elena-Emilia

Opris, Ioana

Orata, Fabini

Orbulov, Imre

Orecchini, Fabio

Oresta, Paolo

Orimoto, Hisako

Orlandi, Antonio

Orlando, Andrea

Orlik-Kożdoń, Bożena

Orłowska-Kowalska, Teresa

Orman, Lukasz

Ornetzeder, Michael

Orosa, José A.

Orosz, György

Orosz, Tamás

Orsagova, Jaroslava

Ortar, Nathalie

Ortenzi, Fernando

Ortiz, Francisco Javier Gutiérrez

Ortjohann, Egon

Orupõld, Kaja

Orynycz, Olga

Osán, János A.

Osara, Jude

Osatiashtiani, Amin

Oshman, Christopher

Osipowicz, Tomasz

Oskarbski, Jacek

Oskay, Rıza Görkem

Osman, Ahmed

Osobajo, Oluyomi A.

Osório, Wislei R.

Ospina, Juan

Osselin, Florian

Ossowicz, Paula

Ostadhassan, Mehdi

Ostanek, Jason

Ostasevicius, Vytautas

Oster, Kamil 
Østergaard, Martin B.

Östlund, Stefan

Ostrauskaite, Jolita

Otanicar, Todd

Otero, José A.

Otmačić Ćurković, Helena

Otomański, Przemysław

Ots, Katri

Otsuka, Akihiro

Otsuki, Akira

Ottaviano, Andrea

Otter, John

Otto, Andreas

Ottonelli, Massimo

Ou Yang, Hung-Yen

$\mathrm{Ou}$, Qisheng

Ouakad, Hassen

Ouamara, Daoud

Ouattara, Osman

Ouchen, Sabir

Ouellet-Plamondon, Claudiane

Ouf, François-Xavier

Ougier-Simonin, Audrey

Ougolnitsky, Guennady

Ould El Moctar, Ahmed

Oureilidis, Konstantinos

Ouro Barba, Pablo

Oussar, Yacine

Outbib, Rachid

Outeiro, Maria Teresa

Ouyang, Xiaolong

Ouzilleau, Philippe

Ovaere, Marten

Ovalle-Encinia, Oscar

Ovalles, Cesar

Ovando, Gustavo

Overbye, Thomas J.

Owen, Herbert

Oyarbide, Mikel

Oyedele, Akinola

Oyewunmi, Oyeniyi

Ożadowicz, Andrzej

Ozarisoy, Bertug

Ozawa, Akito

Ozbayoglu, Evren

Ozer, Ekin

Ozkan, Aylin

Ozkan-Aydin, Yasemin

Oztan, Cagri

Pääkkönen, Anna

Pablo-Romero, María
Pacana, Andrzej

Pach, Grzegorz

Pacheco, João

Pacheco, Pedro Manuel Calas Lopes

Pachla, Filip

Pachta, Vasiliki

Paciorek-Sadowska, Joanna

Pacławski, Adam

Pacurar, Claudia

Padilla-Rivera, Alejandro

Padmanaban, Sanjeevikumar

Padmanabhan, Venkat

Padovan, Dario

Paez-Hernández, Ricardo

Paganin, Valdecir Antonio

Pagano, Alessandro

Paganucci, Fabrizio

Pagger, Ernst

Pagliarini, Giorgio

Pagnini, Luisa

Pagnotta, Stefano

Pagone, Emanuele

Pai, Kai Jen

Pai, Kedar

Paice, Andrew

Paik, Ungyu

Pailleret, Alain

Pająk, Leszek

Pająk, Michał

Pajdak, Anna

Pakala, Sri Harsh

Pakhomov, Maksim

Pal, Anamitra

Pal, Pinaki

Palade, Tudor

Palazoglu, Ahmet

Palazzo, Pierfrancesco

Palczewski, Jan

Palczynska, Beata

Palella, Boris Igor

Palencia, Juan C. Gonzalez

Paleu, Viorel

Palevicius, Arvydas

Palis, Stefan

Pałka, Piotr

Palko, James

Palkova, Zuzana

Palladino, Domenico

Pallarès, David

Pallonetto, Fabiano

Palm, Jenny 
Palmer, Diane

Palmieri, Fulvio

Palmieri, Marco

Palo, Emma

Palomares-Salas, José Carlos

Palomba, Valeria

Palone, Francesco

Palos, Roberto

Palos-Sanchez, Pedro

Pálsson, Ólafur Pétur

Paluch, Andrew S.

Palumbo, Annunziata

Palumbo, Elisabetta

Paluszczyszyn, Daniel

Pályi, Béla

Pamucar, Dragan

Pan, Chengfeng

Pan, Chi-Ling

Pan, Dingyi

Pan, Haozhi

Pan, Liang

Pan, Pengmin

Pan, Quanwen

Pan, Shu-Yuan

Pan, Wei-Ping

Pan, Yang

Pană, Adrian

Panagakos, George

Panagiotelis, Anastasios

Panagiotis, Tsiakaras

Panagoulia, Dionysia

Panait, Mirela

Panão, Marta J. N. Oliveira

Panapakidis, Ioannis

Panaras, Giorgos

Panchenko, Vladimir

Pancik, Juraj

Panda, Biranchi

Pandare, Aditya K.

Pandey, Pramod

Pandit, Naba Raj

Pandit, Ravi

Pandya, Bhargav

Pandyaswargo, Andante Hadi

Pandžić, Hrvoje

Panepinto, Deborah

Pang, Da-Chen

Pang, Shusheng

Pangborn, Herschel

Pani, Shantanu Kumar

Panić, Branislav
Panico, Antonio

Panizio, Roberta M.

Panoiu, Manuela

Pantaleo, Antonio

Panthi, Dhruba

Panthi, Krishna

Pantoli, Leonardo

Panwar, Mayank

Panzarasa, Guido

Paolella, Andrea

Paolillo, Gerardo

Paolini, Riccardo

Paolini, Valerio

Paolone, Annalisa

Paoloni, Stefano

Paone, Emilia

Pap, József

Papa, Ivica

Papacek, Stepan

Papadakis, Raffaello

Papadimitriou, Fivos

Papadopoulos, Agis

Papadopoulos, Athanasios

Papadopoulos, Gerassimos A.

Papadopoulos, Theofilos

Papageorgas, Panagiotis

Papageorgiou, George Z.

Papagiannakis, Roussos

Papaioannou, George P.

Papalambrou, George

Papalou, Angeliki

Papanikolaou, Nick

Papanikolaou, Seraphim

Papanikou, Maria

Papari, Behnaz

Papathanassiou, Stavros

Papatsimpa, Charikleia

Papavasileiou, Georgios

Papavasiliou, Joan

Papayiannis, Georgios

Papoutsidakis, Michail G.

Pappas, Stelios

Papurello, Davide

Paquete, Catarina M.

Parada Medina, Raúl

Paramonov, Alexander

Paraschivoiu, Marius

Pardo Picazo, Miguel Ángel

Paredes, José Pablo

Pareschi, Fabio

Parfenov, Evgeny 
Parikh, Pritesh

Parisi, Alfio

Parisi, Filippo

Parisi, Maria Laura

Parisse, Jean-Denis

Park, Byungkwon

Park, Chan-Bae

Park, Changhyup

Park, Daeryong

Park, Gisu

Park, Haejun

Park, Hanwook

Park, Heesung

Park, Hojeong

Park, Ilchung

Park, Ilhwan

Park, Inhwan

Park, Jaehyun

Park, Jin Chul

Park, Jin-Soo

Park, Ji-Won

Park, Jiyoung

Park, Joong Yull

Park, Ki-Bum

Park, Kyung-Soon

Park, Kyungtae

Park, Min

Park, Sangki

Park, Suhan

Park, Sunho

Park, Taehyun

Park, You-Jin

Park, Young-Jin

Park, Young-Kwon

Parker, Gordon G.

Parker, Max

Parker, Nathan

Parlange, Marc

Parnian, Mohammad Javad

Parobek, Jan

Parrish, Kristen

Parrondo-Gayo, Jorge Luis

Parsazadeh, Mohammad

Parsegov, Sergei

Pärssinen, Matti

Partoon, Behzad

Parupudi, Tejasvi

Parus, Arkadiusz

Parv, Luminita

Parvez, Imtiaz

Parzentny, Henryk R.
Paś, Jacek

Pascal, Carlos

Pascal, Rémy Claude René

Pascual-Muñoz, Pablo

Paseka, Stanislav

Pasetti, Marco

Pashchenko, Dmitry

Pašić, Borivoje

Pasini, Gianluca

Paska, Józef

Pasławska, Marta

Pasman, Hans J.

Pasquale Giofrè, Vincenzo

Pasti, Luisa

Pastore, Nicola

Patalas-Maliszewska, Justyna

Patarau, Toma

Patel, Harshkumar

Patella, Domenico

Patelli, Edoardo

Pater, Sebastian

Paterson, Nigel

Pathak, Rajesh

Pathan, Tanveerkhan

Pathmanathan, Mehanathan

Patil, Sachin

Patil, Sandeep

Patil, Swati J.

Patil, Vikram

Patiño, David

Patlakas, Panagiotis

Patra, Sandipan

Patriarca, Riccardo

Patruno, Luca

Patsalides, Minas

Patuzzi, Francesco

Pau, Giovanni

Paul, Sanjoy

Paul, Satyam

Paul, Seema

Paul, Shuva

Paul, Subhash

Paul, Suvash Chandra

Paul, Titan C.

Paul, Varun

Paul-Boncour, Valérie

Paulenco, Anca

Pauliková, Alena

Paun, Dragos

Pauss, Andre

Pavaloaia, Vasile Daniel 
Pavan Kumar, B. N.

Pavel, Octavian Dumitru

Pavelka, Michal

Pavić, Ivan

Pavić, Ivica

Pavić, Luka

Pavlas, Martin

Pavle, Jakovac

Pavlenko, Ivan

Pavlica, Egon

Pavlov, Nikolay

Pavlyuk, Dmitry

Pawitan, Krisna

Pawliczek, Roland

Pawłowicz, Bartosz

Pawlowski, Andrzej

Pawłowski, Eligiusz

Pawlowski, Lucjan

Paykani, Amin

Payne, James E.

Payne, Vance William

Paz-Ferreiro, Jorge

Pazzi, Veronica

Peakman, Aiden

Pearce, Joshua M.

Pearce, Prafula

Pearsall, Nicola

Peces, César Benavente

Pech Cervantes, Andres Alfredo

Pechanek, Roman

Pecht, Michael Gerard

Pecorini, Isabella

Pedcenko, Alex

Peddapuram, Adithya

Pedicini, Rolando

Pedreño Rojas, Manuel Alejandro

Pedro, Rodríguez-Ayerbe

Pedrosa, Delfim

Peer, Akshit

Peffer, Therese

Pegalajar Jiménez, María Del Carmen

Pehlken, Alexandra

Pei, Yanzhong

Peimankar, Abdolrahman

Peixoto, Andreia F.

Pekař, Miloslav

Pelau, Corina

Pelegov, Dmitry

Pelicano, Christian Mark

Pelin, Denis

Pelinescu, Elena
Pelissier, Serge

Peljo, Pekka

Pellegrini, Marco

Pellegrini, Riccardo

Pellegrino, Roberta

Peltan, Tomáš

Peluso, Valentino

Pembroke, J. Tony

Peña, Araceli

Peña, Rafael

Peña-Cruz, Manuel I.

Peña-García, Antonio

Peña-Pitarch, Esteban

Peñarrocha, Ignacio

Penchev, Hristo

Pencheva, Tania

Peng, Ming-Tsan

Peng, Tongrui

Peng, Yarui

Penkova, Nina

Penkuhn, Mathias

Penz, Florian Markus

Peppas, Georgios

Perales Jarillo, Mikel

Perales, Jose Manuel

Peralta, M. Estela

Perano, Mirko

Perduková, Daniela

Perea Moreno, Alberto Jesús

Perea-Moreno, Miguel-Angel

Perec, Andrzej

Perego, Rodolfo

Pereira Costa, Dulce Helena

Pereira, Adelino

Pereira, Amaro Olimpio

Pereira, António

Pereira, Filipe S.

Pereira, Guillermo Ivan

Pereira, Pedro

Pereira, Pedro F.

Pereira, Rita

Pereira, Rui

Pereira, Sandra

Perera, Noel

Perera, Piyaruwan

Peretto, Lorenzo

Pereyra, Eduardo

Pérez Andreu, Víctor

Perez De Gracia, Fernando

Pérez De Prado, Rocío Josefina

Pérez Menéndez, Ramón José 
Pérez Rodríguez, Sara

Pérez, Agustın-Agüera

Pérez, Alberto Laso

Pérez, Isidro A.

Pérez, Luis

Pérez-Collazo, Carlos

Pérez-García, Vicente

Pérez-Grande, Isabel

Pérez-Hernández, Francisco

Pérez-Miñana, Elena

Pérez-Orozco, Raquel

Pérez-Rey, Ignacio

Pérez-Rodríguez, Martin

Pérez-Ruiz, Juan

Pérez-Sánchez, Modesto

Periša, Marko

Perissi, Ilaria

Perkumienè, Dalia

Perlikowski, Przemysław

Pernía, Alberto M.

Pernigotto, Giovanni

Peron, Fabio

Perote, Javier

Perpiñà, Xavier

Perras, Matthew A.

Perrella, Michele

Perrin, Lara

Personal, Enrique

Persson, Mattias

Pescetelli, Sara

Pesch, Alexander $\mathrm{H}$.

Pescini, Elisa

Pestov, Alexander

Petala, Athanasia

Peter Amalathas, Amalraj

Peter, Laszlo

Peters, Ralf

Petit-Boix, Anna

Petley, Sean

Petnga, Leonard

Petracchini, Francesco

Petrakis, Evangelos

Petran, Horia

Petrella, Andrea

Petrescu, Catalin

Petrescu, Lucian

Petreus, Dorin

Petrichenko, Lubov

Petrik, Máté

Petrillo, Antonella

Petritoli, Enrico
Petrollese, Mario

Petrone, Giovanni

Petropoulos, Spyridon

Petroșanu, Dana-Mihaela

Petrou, Kleoniki Natalia

Petroutsatou, Kleopatra

Petrov, Arthur I.

Petrova, Mariana

Petrova, Tatiana

Petrovic, Goran

Petrovic, Ivica

Petrović, Jovana

Petru, Livinti

Petrunin, Ivan

Pettersson, Frank

Pettinger, Karl-Heinz

Petuhov, Oleg

Peuportier, Bruno

Peuteman, Joan

Pevec, Dubravko

Pevzner, Roman

Pexa, Martin

Peyman, Mohammadmoradi

Peyman, Razi

Pezzinga, Giuseppe

Pezzolla, Daniela

Pezzuolo, Andrea

Pezzutto, Simon

Pfeifer, Antun

Pfleger, Jiri

Pfost, Heiner

Phaal, Rob

Pham, Ngoc Thanh

Pham, Phuong

Phan, Hoang-Phuong

Phan, Linh

Phan, Manh-Huong

Philipsen, Ralf

Phillips, Caleb

Phillips, Jonathan

Phipps, Robyn

Piana, Giulia

Piancastelli, Luca

Piano, Andrea

Picallo-Perez, Ana

Piccardo, Paolo

Picchi, Alessio

Picchio, Rodolfo

Pichon, Lionel

Pickering, Bryn

Piechna, Janusz 
Piechowiak, Tomasz

Piekut, Marlena

Pielecha, Jacek

Piemonte, Vincenzo

Pienkowski, Krzysztof

Pieper, Jeff

Pierdominici, Simona

Pierella, Fabio

Pierleoni, Paola

Pieta, Izabela S.

Pietrasanta, Luca

Pietraszek, Jacek

Pietrowicz, Slawomir

Pietrowski, Wojciech

Pietruschka, Dirk

Pihera, Josef

Pijarski, Pawel

Pikuła, Dorota

Pilar González Vázquez, María

Pilarczyk, Marcin

Pilarska, Agnieszka A.

Piłatowska, Mariola

Pilecki, Zenon

Pili, Roberto

Pillai, Ajit C.

Pillai, Dhanup

Pillai, Gobind Gopalakrishna

Piller, Marzio

Pilloni, Virginia

Pimonenko, Tetyana

Pinamonti, Piero

Pinar-Pérez, Jesus

Pinceti, Paolo

Pindozzi, Stefania

Pineda, Salvador

Pineda-Sanchez, Manuel

Piñerez Torrijos, Iván Darío

Pingitore, Nicholas

Pinheiro, Plácido

Pinho, Carlos

Pinho, Henrique J. O.

Pinho, João

Pinho, Luís

Pinilla, Sergio

Pinion, Clint

Pinnarelli, Anna

Pinon Pereira Dias, Joao Victor

Pintarič, Zorka Novak

Pintauro, Peter N.

Pinter, Gabor

Pinterić, Marko
Pintilii, Radu-Daniel

Pinto, Lígia Maria

Pinto, Manuel

Pinto, Sonia Ferreira

Piot-Lepetit, Isabelle

Piotrowski, Paweł

Piotto, Massimo

Piperopoulos, Elpida

Piqueras, Pedro

Piquet, Hubert

Piras, Giuseppe

Pires Manso, Jose' Ramos

Pires, Armando José Pinheiro Marques

Pires, Fernão V.

Pires, Luis Carlos Carvalho

Pires, Vitor Fernão

Piringer, Martin

Pirnia, Mehrdad

Pirogov, Sergey

Pirouz, Behrouz

Pisano, Giuditta

Pisello, Anna

Piskorski, Łukasz

Piskunov, Maxim

Pislaru-Danescu, Lucian

Píštěk, Václav

Pistikopoulos, Stratos

Pisupati, Sarma V.

Pisz, Iwona

Pitas, Charalampos N.

Pitel, Ján

Pitto, Andrea

Piva, Stefano

Piwowar, Arkadiusz

Piwowarski, Marian

Pizzuti, Stefano

Plachetka, Ulrich

Plakandaras, Vasilios

Platero, Carlos A.

Plathottam, Siby Jose

Platzer, Warner

Plavsic, Tomislav

Plaza, Marta González

Plebankiewicz, Edyta

Pleşca, Adrian

Plesiński, Karol

Pliatsios, Dimitrios

Plöchl, Matthias

Plotkin, Juriy

Plotnikov, Leonid

Pnevmatikos, Nikos 
Poch, Manel

Pocol, Cristina Bianca

Pode, Ramchandra B.

Podgorelec, Blaž

Podgornovs, Andrejs

Podkovalnikov, Sergei

Podporkin, Georgij V.

Podrug, Srdjan

Poerschke, Ute

Pogacar, Tjasa

Poganietz, Witold-Roger

Poggi, Francesca

Poggi, Jean-Michel

Poggi, Philippe

Poguluri, Sunny Kumar

Pohl, László

Poinssot, Christophe

Polach, Pavel

Polajžer, Boštjan

Polak, Adam

Polak, Krzysztof

Polák, Martin

Polanski, Marek

Polanský, Radek

Polatidis, Nikolaos

Polenghi, Adalberto

Poliakov, Nikolai

Policicchio, Alfonso

Polimeni, Antonio

Polinori, Paolo

Polishuk, Ilya

Poljak, Igor

Pollitt, Michael

Pollman, Anthony G.

Połom, Marcin

Polverino, Pierpaolo

Polyanskiy, Mikhail

Polyansky, Oleg P.

Pompeu, João

Poncela, Marta

Poniewiera, Marian

Poniewski, Mieczysław

Pönkä, Harri

Ponnaganti, Pavani

Pons, Josefina

Pons, Oriol

Ponsiglione, Cristina

Pont, Ulrich J.

Ponta, Linda

Pontelli, Enrico

Poolla, Chaitanya
Poom, Age

Pooranian, Zahra

Pop, Claudia Violeta

Pop, Horia F.

Pop, Ioan

Pop, Lucian - Cristian

Popa, Ana-Adela

Popa, Bogdan

Popa, Dan-Cristian

Popa, Gabriel Nicolae

Pop-Calimanu, Ioana-Monica

Popelka, Stanislav

Popescu, Mihaela

Popescu, Vlad

Poplawski, Tomasz

Popli, Nipun

Popov, Marjan

Popov, Vladimir

Popovic-Gerber, Jelena

Popp, József

Popp, Till

Poppe, Andras

Porcu, Roberto

Porru, Mario

Porumb, Radu

Posch, Alfred

Poskovic, Emir

Postacchini, Matteo

Postnikov, Ivan

Poulikidou, Sofia

Pouliquen, Mathieu

Poulopoulos, Panos

Pouquet, Annick

Pourakbari Kasmaei, Mahdi

Poure, Philippe

Pouresmaeil, Edris

Pourhashem, Ghadir

Pourhashem, Ghasideh

Pourmousavi Kani, Seyyed Ali

Povinelli, Richard J.

Powell, Tommy

Poxson, David

Poza-Vilches, Fátima

Pozio, Alfonso

Pózna, Anna I.

Pozo Vázquez, David

Pozzebon, Alessandro

Prabaswara, Aditya

Pradhan, Arpan Kumar

Pradhan, Bapi

Pradhan, Kallol 
Pradhan, Prajal

Prager, Maximilian

Prakht, Vladimir

Prakoso, Ari Bimo

Praks, Pavel

Praktiknjo, Aaron

Pramanik, Brahmananda

Prandtstetter, Matthias

Pranggono, Bernardi

Prapotnik Brdnik, Anita

Prasad, Abhnil

Prasad, Gautham

Prashant, Kumar

Prat, Marc

Pratama, Mahardhika

Pratt, Lawrence

Pravalie, Remus

Prażnowski, Krzysztof

Prazuch, Janusz

Precup, Radu-Emil

Predeanu, Georgeta

Prehofer, Christian

Preißinger, Markus

Premachandra, Chinthaka

Premaratne, Malin

Premier, Alessandro

Premrov, Miroslav

Prenc, Rene

Prenosil, Vaclav

Presciutti, Andrea

Prestileo, Fernanda

Prestipino, Mauro

Preston, Daniel J.

Prettico, Giuseppe

Priarone, Antonella

Prieto Herráez, Diego

Prieto, Borja

Prieto, Jesús-Ignacio

Prieto, Juan Luis

Prilandita, Niken

Principi, Paolo

Pritoni, Marco

Priyanto, Dedy Eka

Procesi, Monia

Prochazka, Petr

Prodan, Maria

Proença Brójo, Francisco Miguel Ribeiro

Proenca, Sara

Proesmans, Karel

Profeta, Adriano

Prokop, Ales
Pröll, Tobias

Prończuk, Mateusz

Proskuryakova, Liliana

Proszak-Miąsik, Danuta

Proto, Daniela

Prousalidis, John M.

Prša, Marija

Pruckner, Marco

Pruncu, Catalin I.

Prusek, Stanisław

Prussi, Matteo

Przybylek, Piotr

Przystupa, Krzysztof

Przywara, Artur

Psarros, Georgios N.

Psomopoulos, Constantinos S.

Ptacek, Michal

Ptak, Szymon

$\mathrm{Pu}$, Cong

Puchalka, Radoslaw

Pucher, Ernst

Puchlik, Monika

Puchovsky, Milosh T.

Pudykiewicz, Janusz

Puech, Pascal

Puertas, Antonio M.

Pueyo, Ana

Pugi, Luca

Pugliese, Francesco

Pugnetti, Carlo

Puigdollers, Joaquim

Puitel, Adrian Cătǎlin

Pujol Vazquez, Gisela

Pujol, Toni

Pujol-Nadal, Ramón

Pukalskas, Saugirdas

Pulidindi, Indra Neel

Pulido Arcas, Jesus

Pulka, Jakub

Pullano, Salvatore A.

Pullen, Keith Robert

Pulvirenti, Giulia

Puna, Jaime

Punov, Plamen Borisov

Punzo, Michele

Puranik, Tejas

Purgel, Mihály

Puri, Manas

Pusch, Andreas

Puschita, Emanuel

Pushkarev, Artem 
Pushkarev, Pavel

Puskar, Michal

Puszka, Andrzej

Puszkiel, Julián A.

Putilov, Arcady

Putrus, Ghanim

Putz, Łukasz

Pyakurel, Parakram

Pyra, Józef

Pyrc, Michał

Pyrgioti, Eleftheria

Pyzhev, Anton

Qadri, Muhammad Yasir

Qasem, Mamoun

Qi, Jing

Qi, Zheng

Qi, Zhiyuan

Qian, Jin-yuan

Qian, Xuejun

Qin, Jianjun

Qiu, Robin

Qiu, Zhiyong

Qolomany, Basheer

$\mathrm{Qu}$, Muchao

$\mathrm{Qu}$, Yanbin

Quan, Zhongyi

Quaranta, Emanuele

Quddus, Md Abdul

Qudrat-Ullah, Hassan

Quesada-Pineda, Henry

Quina, Margarida M. J.

Quintero M., Christian G.

Quinto, Ivana

Quirino, Rafael L.

Qyyum, Muhammad Abdul

Rabadán, José A.

Rábago, Daniel

Rabar, Danijela

Rabault, Jean

Rabe, Marcin

Rabelo, Ricardo

Rabiei, Kobra

Rabinovici, Raul

Rabkin, Eugen

Rąbkowski, Jacek

Raceanu, Mircea

Racewicz, Szymon

Rachel, Hoo Poh Ying

Raciti, David

Rącka, Izabela

Rackauskas, Simas
Raclavska, Helena

Rada, Elena

Radanliev, Petar

Radhakrishnan, Nikitha

Radi Ismail, Kamal Abdel

Radica, Gojmir

Radisavljevic-Gajic, Verica

Radočaj, Dorijan

Radon, Jan

Radosavljević, Jordan

Radu, Laura-Diana

Radu, Petru Valentin

Radu, Rosca

Radulescu, Magdalena

Radulescu, Mircea

Radulović, Aleksandra

Radulovic, Jovana

Radwan, Amr Ahmed A.

Radziszewska-Zielina, Elżbieta

Raeis-Hosseini, Niloufar

Rafael Valotta, Rodrigues

Rafael, Silviano

Rafajlovski, Goran

Rafal Damian, Figaj

Rafał, Marcin Laskowski

Rafalskaya, Tatyana

Raffelt, Klaus

Raffo, Antonio

Rafieenia, Razieh

Rafiq H. Siddiqui, Mohammed

Raganati, Federica

Raganati, Francesca

Ragazzi, Marco

Raghavalu Thirumalai, Durai Prabhakaran

Raghavan, Mala

Raghavan, Nagarajan

Ragusa, Antonella

Rahbar, Kiyarash

Rahbari Asr, Navid

Rahbari, Iman

Raheem, Adeeba Abdul

Ráhel', Jozef

Rahil, Abdulla

Rahimi, Mohammad

Rahimi-Gorji, Mohammad

Rahimzadeh, Iman

Rahman, Arifur

Rahman, Ashiqur

Rahman, Khondokar Mizanur

Rahman, Md Mahbubur

Rahman, Md Motiur 
Rahman, Mohammad Azizur

Rahman, Mostaqur

Rahman, Muhib

Rahman, Quazi

Rahmani, Omeid

Rahmanian, Nejat

Rahmati-Abkenar, Mahboubeh

Rahnavard, Rohola

Rai, Alan

Rai, Chandra S.

Raimbault, Juste

Raja Bose, Jefferson

Rajabloo, Talieh

Rajagopal, Deepak

Rajagopalan, Ashwin Kumar

Rajapakse, Athula

Rajapakshe, Chamara

Rajčić, Vlatka

Rajda, Janusz

Rajput, Shailendra

Rajski, Krzysztof

Rajsl, Ivan

Rak, Józef

Rakicka-Pustułka, Magdalena

Rakopoulos, Dimitrios

Rakowska, Aleksandra

Raluca Maria, Aileni

Ramabhotla, Sundari

Ramadan, Haitham Saad Mohamed

Ramadan, Zaher

Ramakrishnan, Sayanthan

Ramallo, Manuel V.

Raman, Gauthama

Ramana, Muvva V.

Ramaraj, Sukanya

Rambabu, Gutru

Ramea, Kalai

Ramírez Díaz, Alfredo

Ramirez, Antonio

Ramirez, Dionisio

Ramirez, Luis

Ramírez-Faz, José C.

Ramírez-Vargas, Carlos A.

Ramón-Cardona, José

Ramos Carvajal, Carmen

Ramos, Ana

Ramos, Célia M. Q.

Ramos, Helena M.

Ramos, Juan Carlos

Ramos, Nuno Manuel Monteiro

Ramos, Pedro
Ramos, Rute

Ramos, Victor

Ramos-López, Darío

Ramos-Real, Francisco Javier

Ramos-Suárez, Juan Luis

Rampa, Vittorio

Rampazzo, Mirco

Ramsey-Musolf, Darrel

Rana, Abu Ul Hassan Sarwar

Ranaee, Ehsan

Ranalli, Giancarlo

Ranalli, Joseph

Randewijk, Peter Jan

Rang, Toomas

Ranganathan, Prakash

Rangarajan, Shriram S.

Rangel, Jose

Rangel, Victor

Rangel-Hernandez, Victor

Ranjula, Bali Swain

Rantuch, Peter

Rao, Dantam

Rao, Jiwu

Rao, U. Mohan

Rapa, Mattia

Rapant, Stanislav

Raposo, Mauro

Raptis, Anastasios

Raptis, Ioannis-Panagiotis

Rasekh, Shahed

Rashedi, A M Mabrur Ahmad

Rasheduzzaman, Md

Rasheed, Eziaku

Rashid Bin Mohd Yusoff, Abd

Rashid, Gilmanur

Rashid, Khalid

Rasmussen, Morten Grud

Rasmussen, Tonny Wederberg

Rasolofondraibe, Lanto

Rasolomampionona, Desire D.

Rasouli, Erfan

Rassõlkin, Anton

Rastogi, Alok

Rata, Gabriela

Ratajczak, Katarzyna

Rathinam, Navanietha Krishnaraj

Rathod, Dhruvang

Ratner, Albert

Rau, Hsin

Rauch, Reinhard

Raud, Merlin 
Raush, Gustavo

Ravaghi, Zohreh

Ravaglioli, Vittorio

Ravelet, Florent

Ravelli, Davide

Ravelli, Silvia

Ravelo, Blaise

Raventós, Oriol

Ravindra (Ravi), Nuggehalli M.

Ravula, Sudhir

Ravyts, Simon

Ray, Ishita

Ray, Richard P.

Rayamajhee, Veeshan

Rayguru, Madan Mohan

Raymond, Jasmin

Razavi, Alireza

Razavi, Mehrdad

Razavykia, Abbas

Razban, Ali

Razzaghpanah, Zahra

Real, Julia

Reale, Fabrizio

Reali, Gianluca

Reatti, Alberto

Rebelo, Carlos

Rebennack, Steffen

Rebizant, Waldemar

Reboud, Christophe

Reczek, Lidia

Reda, Ahmed

Reddy, M. V.

Reddy, Sohail R.

Redel-Macías, María Dolores

Redlarski, Grzegorz

Redondo, Eduardo

Redutskiy, Yury

Rees, Simon

Rees, William E.

Reeves-McLaren, Nik

Refalo, Paul

Reggiani, Susanna

Regueira, Teresa

Regueiro-Ferreira, Rosa María

Rehan, Mohammad

Rehman, Sarish

Reichel, Agata Rudnicka

Reiff-Stephan, Jörg

Reimer, Uwe

Reinap, Avo

Reinbold, Vincent
Reinholds, Ingars

Reinisch, Elena C.

Reinmöller, Markus

Reinsch, Thomas

Reis Benatto, Gisele Alves Dos

Reis, Alberto

Reis, Anabela

Reis, João

Reis, Manuel J. C. S.

Rejdak, Michał

Relvas, Helder

Rem, Peter C.

Remes, Zdenek

Rempel, Alexandra R.

Ren, Guang

Ren, Hao

Ren, Huilong

Ren, Jingzheng

Ren, Yi

Renedo, Carlos J.

Reneses, Javier

Rengarajan, Amirtharajan

Renko, Sanda

Renno, Carlo

Rente, Bruno

Renz, Michael

Renzi, Emiliano

Repecho, Victor

Requier-Desjardins, Denis

Rescia Perazzo, Alejandro

Resende, João S.

Reshetenko, Tatyana

Reshetilov, Anatoly N.

Respondek, Zbigniew

Restuccia, Francesco

Revellame, Emmanuel

Revelou, Panagiota-Kyriaki

Revollar, Silvana

Rey Martinez, Francisco Javier

Rey-Boué, Alexis B.

Reyes Belmonte, Miguel Ángel

Reyes, Miriam

Rey-Hernández, Javier M.

Reynolds, Doug

Rez, Peter

Reza, M. Toufiq

Rezaei Gomari, Sina

Rezaei Rad, Aryan

Rezaeiha, Abdolrahim

Rezania, Shahabaldin

Rezek, Jiří 
Rezić, Tonči

Rezk, Ahmed

Rezk, Hegazy

Rhee, Chang Seop

Rhee, Kyu-Nam

Rhi, Seok-Ho

Rho, Hojung

Riaz, Fahid

Riba Ruiz, Jordi-Roger

Ribal, Agustinus

Ribas, Javier

Ribeiro Nunes, Leonel Jorge

Ribeiro, Ana Isabel

Ribeiro, Jorge T.

Ribeiro, Lucilia

Ribeiro, Luís Frölén

Ribeiro, Thierry

Ribeiro, Tiago

Ribeiro-Soriano, Domingo

Ribič, Janez

Ribickis, Leonids

Riboldi, Luca

Ricard, Ludovic

Ricca, Michela

Ricci, Alessio

Ricci, Daniele

Ricci, Stefano

Ricciardi, Giuseppe

Riccio, Giuseppe

Ricco, Mattia

Riccò, Raffaele

Richa, Kirti

Richards, Robert

Richelli, Anna

Richiedei, Dario

Richter, Marc

Richter, Wolfgang

Richtera, Lukáš

Ridao, Miguel A.

Rider, Andrew N.

Rieke, Roland

Riess, Gisbert

Rietbergen, Martijn G.

Rigas, Emmanouil S.

Riggi, Ezio

Righetti, Giulia

Righini, Davide

Rihar, Andraz

Rikos, Evangelos

Riley, Paul H.

Rill, Georg
Rimkus, Alfredas

Rinaldi, Fabio

Rinaldi, Giovanni

Rinaldi, Stefano

Rinderknecht, Stephan

Rindt, Camilo

Rineiski, Andrei

Ringel, Marc

Ringwood, John

Rinscheid, Adrian

Rios-Aguilar, Sergio

Rippel, Daniel

Riquelme Santos, Jesús Manuel

Risser, Ralf

Rituraj, Rituraj

Riva, Fabio

Rivas, Sandra

Rivera Dominguez, Jorge

Rivera, Sebastian

Rivera, Sergio Raul

Rizzo, Andrea Maria

Rizzo, Gianfranco

Rizzo, Giovanna

Rizzo, Giuseppe

Rizzo, Santi Agatino

Rjoub, Husam

Rman, Nina

Ro, Insoo

Ro, Jong-Suk

Robba, Michela

Robbins, Eleanora I.

Roberto, Corizzo

Roberto, Roberta

Roberts, Daniel

Roberts, Jeremy A.

Roberts, Mike

Roberts, Nicholas

Roberts, Ronald

Robescu, Lacramioara Diana

Robichaud, David

Robinson, Duane A.

Robinson, James

Robinson, Peter

Robles Medina, Alfonso

Robles, Miguel

Robles-Rodriguez, Carlos E.

Robson, Stephen

Robu, Valentin

Rocca, Michele

Rocca, Vera

Roche, Robin 
Rock, Brian

Rock, Michael

Rockstroh, Nils

Rockwood, Donald

Rodemann, Tobias

Rodenas, Pau

Rodger, James

Rodic, Miran

Rodino, Steliana

Rodler, Auline

Rodonò, Gianluca

Rodrigo, Llopis

Rodrigues, Abel

Rodrigues, Alírio

Rodrigues, Cristina Fernanda Alves

Rodrigues, Eduardo M. G.

Rodrigues, Eugénio

Rodrigues, Fernanda

Rodrigues, José Miguel

Rodrigues, Mónica

Rodrigues, Nelson E. V.

Rodrigues, Rafael Valotta

Rodríguez Amenedo, Jose Luis

Rodríguez Fonollosa, Javier

Rodríguez García, Antonio M.

Rodríguez Prieto, Gonzalo

Rodríguez Saiz, Ángel

Rodríguez, Camino Fernández

Rodríguez, Francisco J.

Rodríguez, Jaime

Rodríguez, Javier

Rodriguez, Joaquín

Rodriguez, Noel

Rodríguez-López, Nuria

Rodríguez-Molina, Jesús

Rodríguez-Monroy, Carlos

Rodríguez-Rodríguez, Ignacio

Rodríguez-Sánchez, Jesús

Rodríguez-Soalleiro, Roque

Rodriquez-Fernandez, Jose

Rodway, James

Roeber, Volker

Roekaerts, Dirk J. E. M.

Roelich, Katy

Rogala, Andrzej

Rogala, Zbigniew

Roger, Daniel

Rogers, Eric

Rogers, Juan

Rogers, Tom

Roggo, Dominique
Rogié, Brice

Rogolino, Patrizia

Rogowski, Krzysztof

Rogulj, Katarina

Roh, Changhyun

Rohacs, Jozsef

Rohan, Ali

Rojas, Christian

Rojas, Gabriel

Rojas-Delgado, Brenda

Rokosz, Krzysztof

Rolando, Luciano

Roldán Perez, Javier

Roldan Porta, Carlos

Roldán, Marcelo

Roldán-Blay, Carlos

Rolek, Jaroslaw

Rolewicz-Kalińska, Anna

Rolland, Sam A.

Romagnoli, Manuela

Romagnoli, Silvia

Romagnoni, Piercarlo

Román Gallego, Jesús Ángel

Roman, Adam

Roman, Kibria K.

Roman, Michał

Roman, Monika

Roman, Raul-Cristian

Romanach, Lygia

Romanello, Vincenzo

Romaniuk, Wacław

Romano, Rosa

Romanova, Inna

Romanowicz, Paweł J.

Romanowska-Duda, Zdzisława

Román-Sánchez, Isabel M.

Romanska-zapala, Anna

Romanuke, Vadim

Romanyuk, Yaroslav E.

Romão, Xavier

Romar, Henrik

Romeo, Luis M.

Romerio, Franco

Romero Lázaro, Rubén Augusto

Romero Mora, José Carlos

Romero Navarrete, José Antonio

Romero, David A.

Romero, Francisco P.

Romero, Rosenberg J.

Romero-Cadaval, Enrique

Romuli, Sebastian 
Ronanki, Deepak

Roncevic, Borut

Rondinelli, Concetta

Rong, Ben-Guang

Rong, Guoxin

Rong, Li

Ronga, Domenico

Ronny, Gelleschus

Roper, Courtney

Ropuszyńska-Surma, Edyta

Roque, Antonio

Rosa, Flavio

Rosales Asensio, Enrique

Rosasco, Paolo

Roscia, Mariacristina

Roscow, James

Roselli, Carlo

Rosendahl, Lasse Aistrup

Rosendo-Macías, José Antonio

Roshanmanesh, Sanaz

Rosi, Luca

Rosillo Calle, Frank

Rosina, Elisabetta

Rosinová, Danica

Rosin-Paumier, Sandrine

Roskosz, Maciej

Rosner, Daniel

Ross, Michael

Rossella, Francesco

Rossi, Andreina

Rossi, Edoardo

Rossi, Jessica

Rossi, Mansueto

Rosso, Federica

Rossomando, Francisco

Rostami, Ali Bakhshandeh

Rosu, Stefan George

Roszczyk, Paweł

Rotaru, Andrei

Rothengatter, Werner

Rottengruber, Hermann

Roubík, Hynek

Rouchier, Simon

Rougier, Esteban

Rouleau, Jean

Roulia, Maria

Roumpos, Christos

Roussanaly, Simon

Routh, Prahlad

Routimo, Mikko

Routray, Pratyush
Roux, Charlotte

Rouzbehi, Kumars

Rovella, Natalia

Rovira, Antonio

Rowland, Simon M.

Roy, Abhishek

Roy, Anurag

Roy, Arnab

Roy, Jinia

Roy, Nitin

Roy, Partho Sarothi

Roy, Spandan

Roy, Sukanta

Roy, Sumit

Roy, Swarup

Roy, Tushar Kanti

Royapoor, Mohammad

Royer, Jean-Jacques

Rozakis, Stelios

Różańska, Sylwia

Różański, Zenon

Rozga, Pawel

Różowicz, Sebastian

Rúa, Megan A.

Rubaszek, Michał

Rubiera, Fernando

Rubino, Guido

Rubino, Luigi

Rubino, Sandro

Rubio, Fernando

Rubio-Bellido, Carlos

Rucińska, Joanna

Ruddock, Lloyd W.

Ruderman, Alexander

Rudez, Urban

Rudolf, Pavel

Rudra, Souman

Rudrajit, Mitra

Ruello, Maria Letizia

Rufer, Alfred

Ruffino, Barbara

Rufin, Carlos

Ruggeri, Bernardo

Ruggieri, Gianluca

Ruggieri, Sergio

Ruggiero, Alessandro

Rughoobur, Girish

Rugnini, Lorenza

Rui, Zhenhua

Ruiz, Cristián A. Ducoing

Ruiz, Fredy 
Ruiz, Harold Steven

Ruiz, Héctor A.

Ruiz, Jesús Fernández

Ruiz-García, Alejandro

Ruiz-Gómez, Miguel J.

Ruiz-Rodriguez, Francisco Javier

Runacres, Mark C.

Rundo, Massimo

Ruocco, Concetta

Ruokamo, Enni

Ruoppolo, Giovanna

Ruparathna, Rajeev

Rus, Tania

Rusca, Aura

Rus-Casas, Catalina

Rusconi, Rosella

Rusowicz, Artur

Russo, Angela

Russo, Antonio

Russo, Marianna

Russo, Mario

Rusu, Calin

Rusu, Cristina

Rusu, Eugen

Rusu, Liliana

Rutkowska, Jaroslawa

Ruttert, Mirco

Ruuskanen, Vesa

Ruusunen, Mika

Ruz Ruiz, Mario Luis

Ruz, Francisco

Ružarovský, Roman

Ryabov, Artem

Ryan, Lisa

Rybach, Ladislaus

Rybak, Aurelia

Rybak, Jarosław

Rybak-Niedziolka, Kinga

Rybár, Radim

Rybarczyk, Dominik

Rybarczyk, Piotr

Rydosz, Artur

Rymanov, Alexander

Rymarczyk, Tomasz

Rymarski, Zbigniew

Ryms, Michal

Ryndin, Eugeny A.

Ryndzionek, Roland

Rynkowski, Piotr

Ryńska, Elżbieta

Rysak, Andrzej
Rytöluoto, Ilkka

Ryu, Jaiyoung

Ryu, Jong-hyun

Ryu, Ki-Wahn

Ryu, Kyunghyun

Ryu, Sangin

Ryzhakov, Pavel

Ryzhkov, Sergei

Ryzhov, Alexander

Rząsa, Janina

Rzucidlo, Pawel

Sa'Ed, Jaser

Saadaoui, Jamel

Saadaoui, Safa

Saadat, Mahdi

Saadatnia, Zia

Saakes, Michel

Saari, Jussi

Saavedra, Jorge

Sabater, Bartolome

Sabban, Albert

Sabhahit, Jayalakshmi N.

Sabzehgar, Reza

Saccani, Cesare

Sacchi, Barbara

Sacile, Roberto

Sadati, S. M. Sajed

Sadeq, Dhifaf

Sadi, Mohammad Ashraf Hossain

Sadiki, Amsini

Sadik-Zada, Elkhan Richard

Sadorsky, Perry

Sadraei, Razieh

Sadykov, Vladislav

Šadzevičius, Raimondas

Saeed, Muhammad

Saeed, Nagham

Saeedipour, Mahdi

Sægrov, Sveinung

Saeid Rahimian Koloor, Seyed

Saeidi, Roghayeh

Saeli, Manfredi

Sáez Bastante, Javier

Sáez Blázquez, Cristina

Saez, Marc

Safaei, Mohammad Reza

Safari, Farid

Safari, Mehdi

Safari, Momo

Safari, Nima

Šafarič, Luka 
Safaritirtashi, Mohammadreza

Safdari Shadloo, Mostafa

Saffari Pour, Mohsen

Saffari, Mohammad

Safonov, Alexander Aleksandrovich

Sager, Jalel

Saghi, Hassan

Saghir, Muhammad

Saghir, Ziad

Sagl, Günther

Saha, Bidyut Baran

Saha, Jhantu Kumar

Saha, Suvash

Sahami, Amirreza

Sahin, Atakan

Sahin, Furkan Emre

Sahoo, Deepak

Sahoo, Kamalakanta

Sahoo, Subrat

Sahoo, Sumanta

Sahroni, Taufik Roni

Saint-Drenan, Yves-Marie

Sainz-Gonzalez, Ruben

Saito, Kozo

Sáiz Agustín, Purificación

Saiz, Fernan

Saiz-Álvarez, José Manuel

Sajid, Muhammad

Sajid, Zaman

Sajjadian, Masoud

Sajn, Robert

Sakagami, Kimihiro

Sakai, Takeyasu

Sakar, Mohan

Sakata, Yoshitaka

Saket, Mohammad

Sakhabutdinov, Airat Zh.

Sakthivel, Kogularasu

Sala, Simone

Salagean, Tudor

Salahdine, Fatima

Salama, Amgad

Salamone, Francesco

Salas Ruiz, Adela

Salauddin, Md

Salazar, Mauro

Salceanu, Alexandru

Salceda-Delgado, Guillermo

Saleem, Arslan

Saleem, Muhammad

Salehfar, Hossein
Salehi, Mohammadsadegh

Salehi, Saeed

Salerno, Nunzio

Salewski, Mirko

Salgado, R.

Salgansky, Eugene Aleksandrovichn

Šalić, Anita

Salim, Muath Bani

Salina Borello, Eloisa

Salkuti, Surender Reddy

Salm, Cora

Salmas, Constantinos E .

Salmerón Lissén, José Manuel

Salom, Jaume

Salomon, Camila P.

Salomone, Roberta

Saloux, Etienne

Salvadori, Giacomo

Salvadori, Simone

Salvalai, Graziano

Salvati, Luca

Salvini, Coriolano

Salvo Rossi, Pierluigi

Saly, Vladimir

Salzano, Ernesto

Samaddar, Subhajyoti

Samadi, Sascha

Samaniuk, Joseph R.

Samaranayake, Premaratne

Sameti, Mohammad

Sampaio, Carlos Hoffmann

Sampoli, Maria Lucia

Samsun, Remzi Can

Samuel, Edmund

Sanabria, Janeth

Sanada, Kazushi

Sanaei, Pejman

Sanchez De La Flor, Francisco José

Sánchez García-Vacas, Daniel

Sanchez- Gonzalez, Alberto

Sanchez Iranzo, Javier

Sanchez Lasheras, Fernando

Sánchez Sutil, Francisco José

Sanchez, Alejandro

Sánchez, Antoni

Sánchez, Antonio Bernardo

Sánchez, Antonio L.

Sánchez, Bordel

Sanchez, Daniel

Sánchez, René-Vinicio

Sanchez-Cambronero, Santos 
Sanchez-Carmona, Alejandro

Sánchez-Fernández, José Ángel

Sánchez-Fernández, María Dolores

Sánchez-Moral, Simón

Sánchez-Rivas García, Javier

Sanchez-Ruiz, Alain

Sánchez-Velasco, Francisco Javier

Sandborn, Peter

Sanderse, Benjamin

Sandholz, Simone

Sandhu, Hardev S.

Sandou, Guillaume

Sandoval Goes, Luiz Carlos

Sandoval, Alicia Bayón

Sandoval-Herazo, Luis Carlos

Sandrolini, Leonardo

Sanduleac, Mihai

Sanei, Seyed Hamid Reza

Sang, Yuanrui

Sani, Luca

Sanjari, Mohammad

Sanjeet, Mehariya

Sanjou, Michio

Sanjurjo-Sánchez, Jorge

Sankaran, Ganapathy Subramanian

Sankarasubramanian, Shrihari

Sanmiquel, Lluís

Sannino, Raniero

Sansom, Chris

Sansoni, Paola

Santamaria, Laura

Santangelo, Paolo E.

Šantek, Mirela Ivančić

Santhanam, KSV

Santi, Giovanni

Santiago Carretero, Oscar

Santiago Chiquero, Isabel

Santiago, Molina

Santillán, David

Santiso, Cristian Ferreiro

Santoni, Andrea

Santoro, Ilaria

Santoro, Sergio

Santos Pérez, Carlos

Santos, Carla

Santos, Cleis

Santos, José

Santos, José Ignacio

Santos, Maria Jesus

Santos, Paulo

Santos, Rafael M.
Santos, Ricardo

Santos, Ronaldo G.

Santos, Vitor

Santos-Reyes, Jaime

Santucci, Valentino

Sañudo, Roberto

Sanvito, Francesco Davide

Sanyal, Udishnu

Sanz, Teresa

Sanz-Gorrachategui, Iván

Sanz-Hernández, Alexia

Sapena-Baño, Angel

Sapin, Paul

Sapińska-Śliwa, Aneta

Saprykina, Yana

Sarafidis, Yannis

Sarafraz, Mohammad Mohsen

Sarajcev, Petar

Sarakis, Lambros

Saralegui, Unai

Saratale, Ganesh Dattatraya

Sarauer, Jessica

Saravanakumar, Kandasamy

Sardi, Alberto

Sardianou, Eleni

Sari, Dany Perwita

Sarjas, Andrej

Šarkan, Branislav

Sarkar, Biswajit

Sarkar, Nurul I.

Sarker, Debalaya

Sarker, Shiplu

Sarker, Subrata

Sarlis, Nicholas

Sarnè, Giuseppe Maria Luigi

Sarniak, Mariusz

Sarout, Joel

Sarris, Ernestos

Sarris, Ioannis E.

Sartori, Luca

Sarwar, Suleman

Sasajima, Yasushi

Sasaki, Daisuke

Sasaki, Tohru

Saseendran, Vishnu

Sasi, Nayar

Sasiadek, Jurek

Sasiadek, Michal

Sasmito, Agus Pulung

Sassanelli, Claudio

Sassi, Mohamed 
Sasso, Maurizio

Sassu, Mauro

Sata, Noriko

Satchwell, Andrew

Sathya, Vanlin

Sathyan, Shelas

Sato, Hiroyuki

Satoshi, Irei

Satpathi, Kuntal

Satpute, Nitin

Sattarpanah Karganroudi, Sasan

Sattary, Sattar

Sattinger, Walter

Sauer, Nathalie

Sauhats, Antans

Saunders, Harry D.

Saunoris, James

Sava, Alexandre

Sava, Gabriela

Savabeghi, Mehdi

Savaghebi, Mehdi

Savaglio, Claudio

Savard, Christophe

Savino, Giovanni

Savino, Stefano

Savkin, Andrey V.

Savkovic, Borislav

Savoldi, Laura

Savuto, Elisa

Savvidou, Maria

Saxen, Henrik

Saxena, Saurabh

Sayed, Ehab

Sayed, Mohamed

Sayegh, Marderos

Sbrizzai, Roberto

Scajev, Patrik

Scala, Barbara

Scalera, Lorenzo

Scamarcio, Alessandro

Scamardella, Antonio

Scandurra, Giuseppe

Scarcelli, Riccardo

Scarfone, Antonio

Schabowicz, Krzysztof

Schade, Gunnar W.

Schadt, Martin

Schaefer, Jennifer

Schaeffer, Emmanuel

Schäfer, Helmut

Schaffarczyk, Peter
Schanen, Jean-Luc

Schatz, Markus

Schaumann, Peter

Scheepers, Fabian

Scheffe, Jonathan

Scheidl, Rudolf

Schell, Kristen

Scheller, Fabian

Schelly, Chelsea

Schenk, Johannes

Schenkendorf, Rene

Schepelmann, Philipp

Schettino, Giuseppe

Schiavone, Giuseppe

Schicker, Irene

Schimmel, Saskia

Schimmelmann, Arndt

Schimpe, Michael

Schirmer, Pascal

Schirone, Luigi

Schito, Eva

Schladitz, Katja

Schlegel, Steffen

Schlosser, Viktor

Schmeck, Hartmut

Schmid, Jan Fabian

Schmitt, Douglas

Schmitz, Gerhard

Schmitz, Katharina

Schneider, Jens

Schneider, Marius

Schnotale, Jacek

Schobert, Harold H.

Schoenefeld, Jonas

Schoenwald, David

Scholle, Markus

Schönfeldt, Patrik

Schönheit, David

Schottroff, Felix

Schrader, Wolfgang

Schrefler, Bernhard

Schreiber, Andrea

Schriemer, Henry

Schröder, Elisabeth

Schrötter, Martin

Schubert, Sebastian

Schuett, Reiner

Schuldt, Steven

Schülein, Erich

Schullerus, Gernot

Schultz, Heyko Juergen 
Schultz, Richard A.

Schulz, Detlef

Schulz, Nicola

Schulz, Wolfgang H.

Schumacher, Walter

Schumayer, Daniel

Schunert, Sebastian

Schuß, Matthias

Schutz, Jérémie

Schwanitz, Valeria Jana

Schwarz, Philip

Schwede, Dirk

Schweiger, Hans-Georg

Schweighofer, Bernhard

Schweiss, Ruediger

Schweizer, Thomas

Schwenzel, Julian

Schwiegelshohn, Fynn

Sciarretta, Antonio

Sciubba, Enrico

Sciuto, Grazia Lo

Scobie, James A.

Scoccia, Rossano

Scordia, Danilo

Scorpio, Michelangelo

Scorrano, Mariangela

Scotognella, Francesco

Scott, Alison

Scott, Daniel M.

Scott, Lloyd

Scott, Paul

Scott, Samuel W.

Scott, Stuart

Scotti, Ivano

Scribano, Vittorio

Scungio, Mauro

Scuro, Carmelo

Scutaru, Maria Luminita

Sdanghi, Giuseppe

Sean, Wu-Yang

Seay, Jeffrey R.

Sebastian Giraldo, Juan

Sebastian, Verhelst

Sechilariu, Manuela

Sedlacek, Petr

Sedlar, Milan

Seduikyte, Lina

Seelam, Prem Kumar

Seeling, Patrick

Seemala, Bhogeswararao

Segundo-Ramírez, Juan
Segura, Francisca

Seh, Zhi Wei

Seib, Matthew D.

Seifan, Mostafa

Sejkorová, Marie

Sęk, Jerzy

Sekioka, Shozo

Sekizaki, Shinya

Self, Ethan

Sellami, Nazmi

Sellers, Ewan

Selopal, Gurpreet Singh

Selosse, Sandrine

Selvaraj, Daisy

Selyanchyn, Roman

Seme, Sebastijan

Semin, Mikhail

Semlitsch, Bernhard

Semmar, Nadjib

Sempere, David Marroquí

Sen, Anando

Sen, Sujat

Sen, Sushobhan

Senčić, Tomislav

Sendin, Alberto

Sendžikienè, Eglè

Sene, Ndolane

Senjyu, Tomonobu

Senneca, Osvalda

Seo, Chulhun

Seo, Dong-Wook

Seo, Hun-Chul

Seo, Hyowon

Seo, Jae-Hyeong

Seo, Kyung-Min

Seo, Myung Won

Seo, Toru

Seo, Youngguk

Seo, Yutaek

Seok, Junhee

Seok, Ogyun

Seong, Nam-Chul

Sepasgozar, Samad M. E.

Sepasi, Saeed

Sepúlveda, Francisco José

Sepulveda-Escobedo, Exequiel

Sequeira, Claudia

Serageldin, Ahmed A.

Serajiantehrani, Ramtin

Serbanoiu, Adrian Alexandru

Seredyński, Mirosław 
Serfa Juan, Ronnie

Sergei, Shtykov

Sergiienko, Nataliia

Seritan, George

Sermet, Yusuf

Sermpinis, Georgios

Sermyagina, Tutkijatohtori Ekaterina

Serpi, Alessandro

Serrà, Albert

Serra, João

Serra, Maria

Serrano Lanzarote, Begoña

Serrano, Angel

Serrano, Antonio

Serrano, Jose

Serrano, Luis

Serrano-fontova, Alexandre

Serrano-Luján, Lucía

Seruga, Przemysław

Servadio, Pieranna

Sescu, Adrian

Sesetty, Varahanaresh

Sessa, Sebastian Dambone

Šestanović, Tea

Sestras, Adriana F.

Sestraș, Paul

Setlak, Lucjan

Setoodeh Jahromy, Saman

Sett, Soumyadip

Sevil, Hakki Erhan

Sevilla, Marta

Sevostianov, Igor

Seyed Ehsan, Hosseini

Seyedaghazadeh, Banafsheh

Seyedzadeh, Saleh

Seyfang, Bernhard

Seyr, Helene

Sferra, Adriana

Sfetsos, Athanasios

Sgouros, Aristotelis

Sgreccia, Emanuela

Sgroi, Mauro Francesco

Sha'aban, Yusuf

Shaaban, Mostafa

Shabani, Babak

Shabaninejad, Mehdi

Shabestari, Parisa M.

Shabgard, Hamidreza

Shabunko, Veronika

Shadike, Zulipiya

Shadloo, Mostafa Safdari
Shafiee, Mahmood

Shafiee, Shahin

Shafiei, Ali

Shafiq, Muhammad

Shafique, Muhammad

Shafiullah, GM

Shafiullah, Md

Shagar, Viknash

Shah, Krishna

Shah, Syyed Adnan Raheel

Shah, Zahir

Shahandeh, Hossein

Shahbazi, Mahmoud

Shaheen, Susan

Shahi, Mina

Shahnia, Farhad

Shahpar, Shahrokh

Shahparasti, Mahdi

Shahri, Abbas Abbaszadeh

Shahrokhi, Ava

Shahzad, Muhammad Wakil

Shakeel, Faiyaz

Shakerighadi, Bahram

Shakor, Pshtiwan

Shaldybin, Michail

Shamberger, Patrick

Shamim, Jubair A.

Shams Ghahfarokhi, Payam

Shamshiri, Redmond R.

Shamsuzzoha, Ahm

Shan, Kui

Shan, Xin

Shang, Chaoqun

Shang, Jun

Shang, Junlong

Shang, Yilun

Shanmugam, Bharanidharan

Shanmugam, Vinodh

Shanyi, Shen

Shao, Chenhui

Shao, Jiankun

Shao, Ling

Shao, Songdong

Shao, Yunli

Shapiro, Jonathan

Sharifi, Amin

Sharifi, Ayyoob

Sharifi, Nourouddin

Sharma, Amit

Sharma, Anurag

Sharma, Arvind 
Sharma, Harmandeep

Sharma, Jyotsna

Sharma, Pushpesh

Sharma, Ram P.

Sharma, Shiv K.

Sharple, Steve

Shateyi, Stanford

Shatnawi, Hashem

Shayesteh Moghaddam, Narges

Shea, John

Sheha, Moataz

Shehu, Rafael

Sheikhnejad, Yahya

Shekaramiz, Mohammad

Shekhar, Aditya

Shen, Bo

Shen, Haoting

Shen, Jingchun

Shen, Sheng

Shenashen, Mohamed A.

Sher, Farooq

Sherizadeh, Taghi

Shevgunov, Timofey

Shew, Aaron

Shewa, Wudneh

Shewarega, Fekadu

Shi, Donglu

Shi, Jian

Shi, Jing

Shi, Qingxin

Shi, Xilin

Shi, Zengqian

Shiau, Steven J. H.

Shie, Je-Lueng

Shieh, Hsin-Jang

Shieh, Jia-Min

Shiga, Seiichi

Shigeishi, Mitsuhiro

Shih, Arthur

Shih, Ming-Cheng

Shimada, Koji

Shimada, Susumu

Shimizu, Makoto

Shimizu, Youichi

Shimoi, Norihiro

Shimojo, Masayuki

Shin, Donghwa

Shin, Donghyuk

Shin, Hyundon

Shin, Jeong-Heon

Shin, Ki-Yeol
Shin, Myunghun

Shin, Seung Gu

Shin, Sunmi

Shin, Tea Ho

Shin, Yoon Hyuk

Shine, Philip

Shintake, Tsumoru

Shipman, Rob

Shirazi, Elham

Shirazi, Saeid Aghahossein

Shirvani Dastgerdi, Ahmadreza

Shirvani Moghaddam, Kamyar

Shishehbor, Mehdi

Shittu, Ekundayo

Shkatulov, Aleksandr

Shmeleva, Nadezhda

Shobande, Olatunji Abdul

Shoeb, Asaduzzaman

Shojaei Barjuei, Erfan

Shojaeighadikolaei, Amin

Shokouhimehr, Mohammadreza

Shorstkii, Ivan

Short, Michael

Shousha, Mahmoud

Shrestha, Lok

Shrestha, Som S.

Shrivastava, Kaustubh

Shtepliuk, Ivan

Shtessel, Yuri B.

Shu, Hung-Yee

Shuaib, Khaled

Shukuya, Masanori

Shun, Yao

Shvetsova, Olga A.

Shwageraus, Eugene

Shyrokau, Bary

Siadatan, Alireza

Siakwah, Pius

Siano, Pierluigi

Sibielak, Marek

Sibilla, Maurizio

Sibilski, Krzysztof

Sica, Francesco

Siddiqui, Osamah

Siddiqui, Sauleh

Sidea, Dorian

Sidiras, Dimitrios

Sidorov, Denis N.

Sidrach, Mariano

Siebenhofer, Matthäus

Sieber, Ingo 
Siedlecka, Urszula

Siedlecki, Rafal

Siegel, Stefan

Siemiątkowski, Piotr

Sieni, Elisabetta

Sienkiewicz, Łukasz

Siergiejczyk, Mirosław

Sierla, Seppo

Sierpiński, Grzegorz

Sierra Fernández, José María

Siew, Renard Yung Jhien

Signoretto, Michela

Siirde, Andres

Sikora, Jan

Sikora, Paweł

Sikorski, Tomasz

Sikorski, Wojciech

Šikšnelytè, Indrè

Silaghi, Helga

Silber, Siegfried

Sileghem, Louis

Silva Llanca, Luis

Silva, Adão

Silva, Alejandro

Silva, André R. R.

Silva, Eliana Costa e

Silva, Fernando

Silva, José

Silva, Manuel Santos

Silva, Susana

Silva-Perez, Manuel

Silvestre, Miguel

Silvestre, Santiago

Silvia, Lorenzo Freire

Silwal, Bishal

Silwal, Sushil

Sim, Hyung Sub

Sim, Sang Jun

Šimáček, Pavel

Simagina, Valentina

Simanaviciene, Zaneta

Simani, Silvio

Šimek, Jiří

Simenfalvi, Zoltan

Simeonov, Ivan

Simić, Srboljub

Simic, Vladimir

Simikić, Mirko

Simion, Cezar Petre

Simionescu, Mihaela

Simo, Anne
Simó, Attila

Simões, Nádia

Simões, Teresa

Simon, Balint

Simon, Christopher A.

Simon, Franz-Georg

Simon, John

Simon, Sebastien

Simonelli, Laura

Simonescu, Claudia Maria

Simonič, Marjana

Simo-Tagne, Merlin

Simpson, Greg D.

Simunovic, Goran

Sinabell, Franz

Sinclair, Anthony N.

Singer, Sigmond

Singh, Abhishek

Singh, David

Singh, Deepak Pratap

Singh, Gurwinder

Singh, Harpreet

Singh, Kamaljit

Singh, Kuldeep

Singh, Madhav

Singh, Mehakpreet

Singh, Nadia

Singh, Raghuveer

Singh, Utkarsh

Singh, Veena

Singsaas, Eric

Sinha Ray, Saikat

Sinha Ray, Sumit

Sinha, Amit K.

Sinha, Jasmine

Sinha, Nityanand

Sinicropi, Adalgisa

Siodla, Krzysztof

Sioshansi, Ramteen

Siozios, Kostas

Siqueira De Carvalho, Ricardo

Siristatidis, Charalampos

Sirodoev, Igor

Siroky, Jaromir

Siroux, Monica

Sirovy, Martin

Sismanis, Panagiotis

Sitko, Dorota

Siu, King Man

Siuta-Olcha, Alicja

Sivák, Peter 
Sivanandam, Sivasankaran

Sivasubramanian, Jayahar

Siwińska, Agata

Sjöblom, Rolf

Skaf, Dorothy W.

Skandalos, Nikolaos

Skarka, Wojciech

Skeie, Nils-Olav

Skiba, Marta

Skillen, Nathan

Skoczko, Iwona

Skoczkowski, Tadeusz

Skoczylas, Norbert

Skok, Srđan

Skok, Srdjan

Skordoulis, Michalis

Skotniczny, Przemysław

Skoulikaris, Charalampos

Skoulou, Vasiliki

Skouras, Eugene D.

Skrickij, Viktor

Skrucany, Tomas

Skrzypacz, Janusz

Skrzypkowski, Krzysztof

Škugor, Branimir

Skunik-Nuckowska, Magdalena

Skvarciany, Viktorija

Sładek, Sławomir

Sladic, Sasa

Slaets, Peter

Slama, Mohammed El Amine

Slanina, Zdenek

Slaoui, Fouad Hasnaoui

Slaski, Grzegorz

Slate, Anthony

Slavinskas, Stasys

Ślefarski, Rafał

Slegers, Nathan J.

Šlégr, Jan

Ślęzak, Radosław

Sliwa, Tomasz

Śliwiński, Paweł

Slocum, Alexander $\mathrm{H}$.

Slowakiewicz, Miroslaw

Słupska, Monika

Ślusarczyk, Beata

Smardon, Richard

Smerek, Lukáš

Smeulders, David

Šmídl, Václav

Smiech, Slawomir
Smierciew, Kamil

Smieszek, Miroslaw

Smit, Gerard J. M.

Smith, Aidan Mark

Smith, Alan

Smith, Edward H.

Smith, Eric

Smith, Erik Robert

Smith, Ian

Smith, Martin

Smith, Paul F.

Smith, Thomas J.

Smoczyński, Piotr

Smokers, Richard

Smoleń, Andrzej

Smoliner, Juergen

Smoliński, Adam

Smołka, Krystian

Smółka, Krzysztof

Smoluk-Sikorska, Joanna

Smuleac, Laura

Smułek, Wojciech

Smulko, Janusz

Smyk, Adam

Smyk, Emil

Snudden, Stephen

So, Byoungjin

So, Hongyun

Soares Dos Santos, Marco P.

Soares, Carlos Guedes

Soares, João

Soares, Nelson

Soares, Tiago

Soava, Georgeta

Sobczak, Izabela

Sobczak, Krzysztof

Sobczynski, Dariusz

Sobianowska-Turek, Agnieszka

Sobieski, Wojciech

Sobola, Dinara

Sobota, Tomasz

Šobra, Jan

Sobrido, Ana Jorge

Sobus, Jan

Sockeel, Nicolas

Soco, Eleonora

Soedarmo, Auzan

Šogenova, Alla

Soh, Chew Beng

Șoimoșan, Teodora Melania

Sokama-Neuyam, Yen Adams 
Sokoloff, Dmitry Dmitrievich

Sokolov, Oleg

Sokolovsky, Vladimir

Sola, Yolanda

Solazzi, Luigi

Soldatenko, Sergei A.

Soldati, Alessandro

Soldatos, Gerasimos T.

Soldo, Jure

Soldo, Vladimir

Soleimani, Majid

Soler, Jaime

Soliman, Abdel-Hamid

Solina, Vittorio

Soliwoda, Michał

Solomin, Evgeny V.

Solomon, Barry

Solouk, Ali

Sołowiej, Piotr

Soltani, Madjid

Soltanian, Reza

Soltaninejad, Mohammadreza

Solteiro Pires, Eduardo José

Sołtysik, Maciej

Šoltysová, Zuzana

Solval, Kevin Mis

Somà, Aurelio

Soma, Gian Giuseppe

Somasundaram, Sivanand

Somma, Renato

Sommerfeldt, Nelson

Somnath, Suhas

Somorin, Tosin

Šomplák, Radovan

Son, Changmin

Son, Hungsun

Son, Jae Sung

Son, Joonwoo

Son, Min

Son, Min-Kyu

Son, Moon

Son, Sung-Yong

Son, Su-Won

Son, Yeongkwon

Sonar, Santosh

Song, Eugene

Song, Hanjung

Song, Hongqing

Song, Jian

Song, Juntae

Song, Meng
Song, Seungho

Song, Seung-Yeong

Song, Simon

Song, Xiaoqing

Song, Yang

Song, Yongze

Song, Yooseob

Song, Young Hoon

Song, Young-Chae

Song, Young-hak

Sonnino, Giorgio

Sontakke, Atul D.

Soo, Vi Kie

Sookhak, Mehdi

Soomro, Abid

Soomro, Mahfooz

Soon, Jong Jeong

Sorandaru, Ciprian

Sorce, Alessandro

Sørensen, Jens

Soriano, Jose A.

Sorohan, Stefan

Sorrentino, Giancarlo

Sorrentino, Marco

Sorrentino, Nicola

Sosnina, Elena

Sosnowski, Marcin

Sotelo Monge, Marco Antonio

Soto Rodriguez, Paul Eduardo David

Soto, Paul

Sotome, Masato

Sottile, Joseph

Soudan, Bassel

Souhan, Brian

Soukis, Konstantinos

Souliotis, Manolis

Souppez, Jean-Baptiste R. G.

Sourbron, Maarten

Sousa Brito, Isabel Sofia

Sousa, Ana

Sousa, Joao Miguel

Sousa, Joaquim

Sousa, Nuno

Sousa, Rita

Sousa, Rita L.

Sousa, Sara

Sousa, Tiago J. C.

Sousounis, Marios Charilaos

Sowa, Jerzy

Sowale, Ayodeji

Sowizdzal, Anna 
Sowiżdżał, Krzysztof

Sowlat, Mohammad H.

Sozańska, Maria

Spaanenburg, Lambert

Spada, Marco

Spadavecchia, Maurizio

Spagnolo, Bernardo

Sparber, Wolfram

Spasiano, Danilo

Spatari, Sabrina

Spătaru, Niculae

Spataru, Sergiu

Spearing, A. J. S. (Sam)

Specht, Eckehard

Speight, James G.

Spencer, Adrian

Spengler, Anderson Wedderhoff

Speretta, Stefano

Šperka, Petr

Sperlì, Giancarlo

Spertino, Filippo

Spiazzi, Giorgio

Špiláček, Michal

Spiliotis, Evangelos

Spiridigliozzi, Luca

Spiridon, Stefan-Ionut

Spitas, Vasilios

Spiteri Staines, Cyril

Spitler, Jeffrey

Spitzer, Karl-Heinz

Špoljarić, Željko

Sprajc, Polona

Spreng, Daniel Theodor

Springer, Martin

Sproul, Alistair

Sreerama, Lakshmaiah

Srinivas, Keerthi

Srinivasamurthy, Sharath

Srinivasan, Bhuvanesh

Sriram, Lalitha Madhavi Konila

Srivastava, Shubham

Srivastava, Vishal

Sroka, Zbigniew J.

Srpak, Dunja

SSennoga, Twaha

Stabauer, Martin

Stabile, Giovanni

Stabile, Luca

Stachowiak, Dorota

Stacul, Stefano

Stadelmann-Steffen, Isabelle
Stadie, Nicholas P.

Stadlober, Ernst

Staffell, Iain

Stafford, Edwin

Stagg-Williams, Susan M.

Staicu, Lucian

Stala-Szlugaj, Katarzyna

Stamatakis, Michael

Stamatelos, Anastassios M.

Stamatelos, Tassos

Stamatin, Ioan

Stamatiou, Anastasia

Stanciu, Camelia

Stanculescu, Marilena

Standar, Aldona

Stănescu, Nicolae-Doru

Stanev, Rayko

Staniek, Marcin

Staninska-Pięta, Justyna

Stanko, Davor

Stanko, Stefan

Stanković, Siniša

Stansby, Peter K.

Starace, Giuseppe

Starikovskiy, Andrey

Starnoni, Michele

Starosta, Roman

Staszczuk, Anna

Stathi, Panagiota

Stathopoulos, Panagiotis

Stauffer, Philip

Stavrakakis, George S.

Stec, Agnieszka

Stechel, Ellen

Steckiewicz, Adam

Steel, Brent S.

Steeneveld, Gert-Jan

Stefani, Giovanni L.

Stefania, Moioli

Stefaniuk, Dawid

Stefanizzi, Michele

Stefanizzi, Pietro

Stefanov, Miroslav

Steffen, Thomas

Stegenta-Dąbrowska, Sylwia

Stegnar, Gasper

Stehel, Vojtech

Steinbach, David

Steinbauer, Miloslav

Steinberg, Spencer

Steinberger-Wilckens, Robert 
Steinhauer, Stephan

Steller, Janusz

Stelson, Kim

Stelzer, Franz

Štemberk, Petr

Stenstrøm, Yngve

Stepenko, Serhii

Stephan, Richard

Stephen, Bruce

Stephens, Thomas

Stepien, Jacek

Stepien, Mariusz

Stergiopoulos, Fotis

Stergiopoulos, Thomas

Stesina, Fabrizio

Stetter, Ralf

Steup, Christoph

Steurer, Elmar

Stevanovic, Ivica

Stevanović, Žana

Stevens, Gunnar

Stević, Željko

Stewart, Brian G.

Stewart, David M.

Stewart, Rodney

Sthel, Marcelo Silva

Stiebler, Manfred

Stipetic, Stjepan

Štirmer, Nina

Stjepanović, Marija

Stluka, Petr

Stoch, Paweł

Stock, Adam

Stoeck, Tomasz

Stoica, Adrian-Mihail

Stoica, Virgil

Stoican, Florin

Stoicuță, Olimpiu

Stoilova, Svetla

Stojceska, Valentina

Stojčić, Nebojša

Stojkov, Marinko

Stolarski, Mariusz

Stoll, Daniel

Stone, Richard

Stopic, Srecko

Stopka, Ondrej

Stopková, Mária

Stoppato, Anna

Storchak, Michael

Stork, Milan
Stornelli, Vincenzo

Storti Gajani, Giancarlo

Stouraiti, Christina

Stovpchenko, Ganna

Stoyanov, Ludmil

Stoyka, Kateryna

St-Pierre, Jean

Strachan, Paul

Strąkowska, Anna

Strąpoć, Dariusz

Stratakis, Antonios

Straub, Jeremy

Stravoravdis, Spyros

Streblow, Rita

Streckiene, Giedre

Streimikiene, Dalia

Strekowski, Rafal

Strelec, Stjepan

Strielkowski, Wadim

Stritih, Uroš

Stritzke, Susan

Striugas, Nerijus

Stroe, Daniel-Ioan

Strohbach, Martin

Ström, Henrik

Struchtrup, Henning

Struková, Zuzana

Strulak-Wójcikiewicz, Roma

Struminska-Parulska, Dagmara I.

Strzałkowski, Karol

Strzałkowski, Paweł

Strzałkowski, Piotr

Strzelecki, Ryszard

Strzemiecka, Beata

Stuart, Thomas

Stuber, Matthew D.

Stubos, Athanassios

Stuckey, David C.

Studzinski, Jan

Stull, Roland

Štumberger, Bojan

Štumberger, Gorazd

Stumpf, Peter

Stuparu, Adrian

Sturm, Bodo

Sturm, Guido

Stürmer, Bernhard

Styczynski, Zbigniew Antoni

Stylianakis, Minas

$\mathrm{Su}$, Chung-Hwei

$\mathrm{Su}$, Jung-Chieh 
Su, Ming-Shou

$\mathrm{Su}$, Xiaojun

$\mathrm{Su}$, Yingchao

$\mathrm{Su}$, Yuehong

Suárez, Eduardo

Suarez, Elisabet

Suárez, Rafael

Suarez-Alcantara, Karina

Suárez-García, Andrés

Suárez-López, María José

Suarez-Rivera, Roberto

Subburaj, Anitha Sarah

Subiantoro, Alison

Subocz, Jan

Subotić, Vanja

Subramaniam, Aravinth

Subramaniam, Umashankar

Subramanian, Chelakara

Subtirelu, Gheorghe-Eugen

Suchorab, Zbigniew

Sudac, Davorin

Sudolska, Agata

Sudzina, Frantisek

Sugahara, Takeshi

Sugano, Masashi

Sugar Gabor, Oliviu

Suh, Jangwon

Suh, Jeongmeen

Suhail Hussain, S. M.

Sui, Dan

Sui, Jay

Sui, Ran

Suicmez, Vural Sander

Sujova, Andrea

Sukič, Primož

Sukin, Ivan

Šulc, Radek

Sułowicz, Maciej

Sultana, Ummul Khair

Sultonov, Mirzosaid

Sum, Yee Loon

Šumak, Boštjan

Sumelka, Wojciech

Summhammer, Johann

Sun, Haiying

Sun, Hongbo

Sun, Kien-Wen

Sun, Li

Sun, Lixin

Sun, Pengnan

Sun, Qiao
Sun, Shichen

Sun, Susan Isaya

Sun, Wei

Sun, Wenqiang

Sun, Xia

Sun, Xiaodong

Sun, Xindi

Sun, Xiuxuan

Sun, Yongxia

Sun, Zeyi

Sundararajan, Aditya

Šunde, Viktor

Sunderland, Keith

Sunderland, Peter

Sundström, Agneta

Sung, Bongsuk

Sung, Guo-Ming

Sungthong, Rungroch

Suntio, Teuvo

Suomalainen, Emilia

Suprun, Emiliya

Suriamin, Fnu

Šurina, Igor

Surowiak, Agnieszka

Surugiu, Maria Claudia

Surup, Gerrit Ralf

Suryawanshi, Mahesh

Suslov, Konstantin

Suslov, Sergey

Susnea, Ioan

Suszanowicz, Dariusz

Sütő, Zoltán

Sutton, Adam T.

Suwała, Wojciech

Suwardi, Ady

Suzdaḷenko, Aleksandrs

Suzuki, Yasushi

Suzuki, Yoshikazu

Svabova, Lucia

Švajlenka, Jozef

Svatos, Jakub

Svecko, Rajko

Svendsen, Svend

Sverbilov, Victor

Svetlana, Tretsiakova-McNally

Svetlík, Jozef

Svidroňová, Mária

Świć, Antoni

Swierc, Ágata

Świercz, Rafał

Świetlicka, Izabela 
Świętochowski, Adam

Swinarew, Andrzej

Świt, Grzegorz

Syed, Ali Muslim

Sylvestre, Alain

Symon, Sean

SYNÁK, František

Synoradzki, Karol

Szabo, Lajos

Szabó, László (Hungary)

Szabó, László (Japan)

Szabo, Lorand

Szabó, Tamás

Szabolcsi, Róbert

Szabova, Zuzana

Szaferski, Waldemar

Szafran, Jacek

Szafranko, Elżbieta

Szajerski, Piotr

Szajt, Marek

Szałowski, Karol

Számel, László

Szarucki, Marek

Szász, Csaba

Szczegielniak, Tomasz

Szczepanek, Marcin

Szczepaniak, Włodzimierz

Szczepanik, Jerzy

Szczepańska, Agnieszka

Szczes, Alexandra

Szcześniak, Paweł

Szczęśniak, Sylwia

Szczypinski-Sala, Wojciech

Szeidert, Iosif

Székely, Edit

Szelag, Adam

Szeląg, Wojciech

Szeleszczuk, Łukasz

Szemes, Pétér Tamás

Szénási, Sándor

Szép, Tekla

Szewczyk, Roman

Szinai, Julia

Szirbik, Nick B.

Szkoda, Mariusz

Szkodo, Marek

Szmyd, Janusz S.

Szodrai, Ferenc

Szopińska, Kinga

Szoplik, Jolanta

Szőri, Milán
Szostek, Małgorzata

Szpica, Dariusz

Szpilko, Danuta

Sztekler, Karol

Sztubecka, Małgorzata

Sztyber, Anna

Sztykiel, Michal

Szufa, Szymon

Szul, Tomasz

Szulc, Piotr

Szulc, Przemyslaw

Szultka, Seweryn

Szumelda, Tomasz

Szurgacz, Dawid

Szwaja, Stanisław

Szwast, Maciej

Szymanda, Jaroslaw

Szymańska, Magdalena

Szymanski, Jerzy R.

Szymborski, Tomasz

Szymczyk, Anna

Szymczyk, Jacek

Szymona, Karolina S.

Szyszka, Jerzy

Szyszkowicz, Mieczysław

Szyszlak-Bargłowicz, Joanna

Ta, Van-Dai

Tabakaev, Roman B.

Tabaran, Flaviu

Tabassum, Shawana

Tabil, Lope G.

Taczanowski, Jakub

Tadanaga, Kiyoharu

Tafarojnoruz, Ali

Taghia, Javad

Taghizadeh, Seyedfoad

Taghizadeh-Hesary, Farhad

Taguchi, Hiroyuki

Tahavori, Maryamsadat

Taher Azar, Ahmad

Taherdangkoo, Reza

Taheri, Pooya

Taherian, Hessam

Tahmas, Faham

Tahmasebi, Pejman

Tahmasebinia, Faham

Tailony, Rauf

Tajani, Francesco

Tajduś, Krzysztof

Tajiri, Kazuya

Takács, Ján 
Takács-György, Katalin

Takahashi, Eiichi

Takahashi, Hidemi

Takahashi, Katsuyuki

Takanori, Hino

Takarics, Béla

Takashima, Toshihiro

Takeya, Satoshi

Takeyama, Akinori

Takyi, Gabriel

Talaat, Ahmed

Talbot, Paul

Talebizadeh Sardari, Pouyan

Taleghani, Sahar Taslimi

Taler, Dawid

Taler, Jan

Taliotis, Constantinos

Tallapally, Venkatesham

Tallini, Marco

Talluri, Lorenzo

Talon, Laurent

Talpur, Saifal

Talukdar, Krishan

Talukdar, Saurav

Tam, Ivan CK

Tamainot-Telto, Zacharie

Tamaru, Yutaka

Tamas Janos, Katona

Tamašauskaitė-Tamašiūnaitè, Loreta

Tamberg, Gert

Tambovceva, Tatiana

Tamer, Aykut

Tamor, Michael A.

Tampio, Nicholas

Tamura, Junji

Tamus, Zoltán Ádám

Tan, Alex Yong Kwang

Tan, Chee-Keong

Tan, Chin Yaw

Tan, Chun Liang

Tan, Hua

Tan, Kuang-Hsiung

Tan, Lei

Tanabe, Yuji

Tanaka, Tadashi

Tanase, Corneliu

Tanasiev, Vladimir

Taner, Tolga

Tang, Chaolong

Tang, Shiang-Feng

Tang, Wallace Kit-Sang
Tang, Xiaochao

Tang, Xin

Tangerås, Thomas

Taniguchi, Shigeru

Taniguchi, Takuya

Tanikawa, Wataru

Tankova, Trayana

Tano, Mauricio

Tansini, Alessandro

Tanveer, Sheik

Tao, Chengcheng

Tao, Jin

Tao, Lei

Taran, Narges

Tarasov, Ivan

Tarczewski, Tomasz

Tarfaoui, Mostapha

Tariq, Muhammad

Tariq, Zeeshan

Tarkowski, Maciej

Tarnapowicz, Dariusz

Tarroja, Brian

Tartakovsky, Leonid

Tarulescu, Radu

Tasco, Vittorianna

Tashpulatov, Sherzod N.

Taslimi Taleghani, Sahar

Tatar, Erdinc

Tateda, Masafumi

Tatomir, Alexandru

Tatsis, Vasileios

Taubert, Lutz

Tauš, Peter

Tauveron, Nicolas

Taveira Pinto, Francisco

Tavin, Bertrand

Tayal, Dev

Taylor, Adam

Taylor, Martin

Taylor, Nathaniel

Taylor, Nigel George

Taylor, Spencer

Tbaileh, Ahmad

Tchagang, Alain

Tchatoka, Firmin Doko

Tchawou Tchuisseu, Batista Eder

Tcvetkov, Pavel

te Heesen, Henrik

Teah, Heng Yi

Tebekaemi, Eniye

Tedesco, Francesco 
Teekaraman, Yuvaraja

Tefferi, Mattewos

Teghil, Roberto

Teh, Ying Khai

Tei, Alessio

Teichert, Olaf

Teijón-López-Zuazo, Evelio

Teimourzadeh Baboli, Payam

Teitel, Meir

Teixeira, José Carlos Fernandes

Teixeira, Senhorinha

Tejedor, Blanca

Tejedor-Flores, Nathalia

Tejero González, Ana

Telejko, Marek

Teleszewski, Tomasz

Teli, Despoina

Telyakovskiy, Aleksey

Teng, Jen-Hao

Teng, Kah Hou

Tenorio, Jose Antonio

Tenorio, Victor Octavio

Tenreiro, Claudio

Teo, How Wei Benjamin

Teo, Nicholas

Teodorescu, Remus

Teodoriu, Catalin

Teodosio, Luigi

Tepljakov, Aleksei

Terry, Stephen D.

Teruel, Enrique

Tescasiu, Bianca

Teschner, Tom-Robin

Tesiero, Raymond C.

Teslyuk, Vasyl

Tesoriere, Giovanni

Teunissen, Peter

Teymouri, Ali

Teyssedre, Gilbert

Thaduri, Adithya

Thakur, Garima

Thakur, Nirmalya

Thakur, Subhasis

Thakur, Vijay Kumar

Thalfeldt, Martin

Tham, Kwok Wai

Thangavel, Sangeetha

Thapa, Rajan

The Vu, Mai

Thengane, Sonal

Theocharis, Andreas
Theodosiou, Theodoros

Theodossopoulos, Dimitris

Theoharis, Babanatsas

Theotokatos, Gerasimos

Theristis, Marios

Thil, Stéphane

Thomaidis, Nikolaos S.

Thomas, Sean

Thomas, Valerie

Thompson, Gregory J.

Thompson, Richard L.

Thompson, Shirley

Thomsen, Leon

Thorarinsdottir, Ragnheidur

Thorpe, David

Thresher, Robert

Thür, Alexander

Tian, Fang-Bao

Tian, Fengchun

Tian, Gui Yun

Tian, Hailin

Tian, Mi

Tian, Wei

Tian, Weisong

Tian, Xiange

Tian, Zhao

Tian, Zhongbei

Tiari, Saeed

Tiba, Chigueru

Tiberiu Petrescu, Florian Ion

Tiberiu, Tudorache

Tic, Wilhelm Jan

Tidwell, Abe

Tien, Ching-Ho

Tien, Chuen-Lin

Tien, Jerry C.

Tietze, Ingela

Tietze-Jaensch, Holger

Tillenkamp, Frank

Time, Rune W

Timon, Rabczuk

Timoshkin, Igor

Tina, Giuseppe Marco

Tinarelli, Roberto

Tinjum, James M.

Tinni, Ali Ousseini

Tinti, Francesco

Tiow, Ooi Kim

Tipantuña, Christian

Tirler, Werner

Tirnovan, Radu-Adrian 
Tisato, Nicola

Tiseira, Andrés Omar

Tishchenko, Victor

Tisma, Marina

Tito, Shafiqur Rahman

Tivanski, Alexei V.

Tkach, Oleksandr

To, Peter

To, Wai Ming

Toader, Dumitru

Toan, Sam

Tochitsky, Sergei

Tochkov, Kiril

Toczylowska-Maminska, Renata

Todeschini, Grazia

Todeschini, Sara

Tofanica, Bogdan Marian

Toffolo, Andrea

Tofoli, Fernando Lessa

Tojo, Tomohiro

Tokarska, Magdalena

Tokede, Olubukola

Toledo, Carlos

Tolj, Ivan

Tolkou, Athanasia

Toma, Lucian

Tomac, Ingrid

Tomalty, Ray

Toman, Petr

Tomanek, Mateusz

Tomar, Anuradha

Tomasiunas, Rolandas

Tomaszewska, Barbara

Tomazzoli, Claudio

Tombros, Stylianos F.

Tomczak, Janusz

Tomczewski, Andrzej

Tomczyk, Krzysztof

Tomić, Tihomir

Tomków, Jacek

Tomm, Jens

Tomoyoshi, Nishimura

Tomporowski, Andrzej

Tompsett, Geoffrey

Tondelli, Simona

Tong, Linyue

Tong, Wenming

Tong, Yen Wah

Tong, Zheming

Tonoli, Andrea

Topel, Monika
Topic, Danijel

Topic, Marko

Topliceanu, Liliana

Topor, Marcel

Toprakci, Hatice Aylin Karahan

Tora, Barbara

Torchio, Marcello

Torchio, Riccardo

Torell, Gregory

Torii, Shuichi

Torikai, Hiroyuki

Tormos, Bernardo

Tornatore, Cinzia

Torok, Adam

Torok, Zoltan

Toroń, Bartłomiej

Torralba, Antonio

Torre, Carmelo Maria

Torres Maldonado, José Francisco

Torres, David

Torres, José A.

Torres, Lizeth

Torrisi, Vincenza

Tortella, Andrea

Torzillo, Giuseppe

Toscani, Andrea

Tostado-Véliz, Marcos

Tosti, Silvano

Tosti, Tomislav

Tosun, Jale

Tota-Maharaj, Kiran

Tóth, Ágota Bányai

Toth, Gergely

Tóth, Tivadar M.

Toubeau, Jean-François

Touloupaki, Eleftheria

Tounsi, Hafssa

Touria, El Mezyani

Tousa, Julia

Towery, Colin

Town, Graham

Townsend, Chris

Toyinbo, Oluyemi

Trafczyński, Marian

Tran, Carolyn

Tran, Quang Anh

Tran, Quynh

Tran, Tan Tai

Tran, Thanh Son

Tran, ThanhToan

Trapanese, Marco 
Traverso, Marzia

Trbušić, Mislav

Tretiakov, Konstantin

Trianni, Andrea

Triantafyllidis, Stavros

Triantafyllos, Tatoulis

Triantis, Dimos A.

Trias, F. Xavier

Tribioli, Laura

Tricase, Caterina

Trif, Monica

Trigo, Juan Francisco

Trigui, Rochdi

Trimboli, Scott

Trinh, Minh Hoang

Trinh, Thuat

Tripathi, Ravi Nath

Tripathy, Sunil Kumar

Trippetta, Fabio

Tristiu, Ion

Trivedi, Japan

Triviño, Alicia

Trkulja, Bojan

Trnka, Pavel

Troian-Gautier, Ludovic

Trojan, Marcin

Trojanek, Radoslaw

Trømborg, Erik

Tronchin, Lamberto

Tronci, Enrico

Trontl, Krešimir

Tropina, Albina

Trovato, Michele

Trovò, Andrea

Trubetskaya, Anna

Truchet, Guillaume

Truchot, Benjamin

Trull, Óscar

Truntič, Mitja

Truong, Nguyen Le

Trybuła, Marcela E.

Trybus, Bartosz

Trygg, Kristina

Tryk, Donald

Trzcinski, Grzegorz

Trzmiel, Grzegorz

Tsai, Chen-Chi

Tsai, Chen-Yen

Tsai, Huan

Tsai, Kang-Ting

Tsai, Kuang-Chung
Tsai, Ming-Jong

Tsai, Ming-Tang

Tsai, Tsuey-lin

Tsai, Wen-Chang

Tsai, Wen-Hsien

Tsai, Wen-Tien

Tsang, Kim Fung

Tsangas, Michail

Tsangrassoulis, Aris

Tsani, Stella

Tsantopoulos, Georgios

Tsatsaronis, Georgios

Tsau, Chun-Huei

Tsau, Jyun-Syung

Tsaur, Ruey-Chyn

Tscharaktschiew, Stefan

Tse, Daniel W. K.

Tse, Ka Kui

Tsekouras, George J.

Tseng, I-Hsiang

Tseng, King

Tseng, Ming-Lang

Tseng, Shu-Ming

Tseng, Yuan-Wei

Tsiakas, Ilias

Tsiamitros, Dimitrios

Tsikaloudaki, Katerina

Tsiligkaridis, John

Tsipouras, Markos G.

Tsiropoulou, Eirini Eleni

Tso, Chi Yan

Tso, Edwin

Tsoka, Stella

Tsolakis, Apostolos

Tsoulfidis, Lefteris

Tsoumanis, Georgios

Tsoutsanis, Elias

Tsoutsos, Theoharis

Tsovilis, Thomas E.

Tsow, Francis

Tsubaki, Koutaro

Tsubaki, Shuntaro

Tsuchiya, Tatsuhiro

Tsuji, Yutaka

Tsujimoto, Yoshinobu

Tsutsui, Waterloo

Tsvetkov, Nikolai

Tsvetkov, Pavel

Tsvetkova, Anastasia

Tsyntsarski, Boyko

$\mathrm{Tu}$, Michael 
$\mathrm{Tu}, \mathrm{Xin}$

Tuchowski, Wojciech

Tucki, Karol

Tudor, Cristiana

Tudoroiu, Nicolae

Tuegeh, Maickel

Tuffner, Frank

Tularam, Gurudeo Anand

Tulbure, Adrian

Tun, Maw Maw

Tuominen, Pekka

Tupa, Jiri

Tupènaitè, Laura

Tupsakhare, Swanand

Turanoglu Bekar, Ebru

Turcios, Ariel E.

Turcu, Antoniu

Turenne, Sylvain

Turner, Jamie

Turren-Cruz, Silver-Hamill

Tusek, Ana Jurinjak

Tusset, Angelo Marcelo

Tutak, Wojciech

Tutueva, Aleksandra

Tutunaru, Lucian

Tuzun, Ugur

Tveit, Svenn

Twary, Scott

Tworek, Katarzyna

Tyliszczak, Bożena

Tymiński, Tomasz

Tyrologou, Pavlos

Tyshchenko, Oleksii

Tywoniak, Jan

Tzabar, Nir

Tzeremes, Panayiotis G.

Tziourtzioumis, Dimitrios N.

Tzouvanas, Panagiotis

Tzu, Fu-Ming

Ubong, Etim

Uceda, Antonio Lopez

Uceda, Javier

Uche Marcuello, Francisco Javier

Uchida, Susumu

Uchida, Tsutomu

Uchida, Yohei

Uchiyama, Kenji

Uchman, Wojciech

Uchroński, Mariusz

Udalov, Oleg

Uddin, Gazi Salah
Udegbunam, John Emeka

Udovichenko, Aleksei

Udris, Dainius

Uemura, Yoshimitsu

Ueno, Satoshi

Ugwu, Johnson

Ugwuanyi, Nnaemeka Sunday

Uhrig, Stephanie

Uibu, Mai

Uihlein, Andreas

Ukrainczyk, Neven

Ulazia, Alain

Ulgiati, Sergio

Ullah, Zia

Ulness, Darin J.

Ulyashin, Alexander G.

Umbleja, Kadri

Ungureanu, Constantin

Unich, Andrea

Upare, Pravin P.

Upham, Jeremy

Upreti, Simant

Uprety, Prakash

Upton, John

Úradníček, Juraj

Urata, Junji

Urbancl, Danijela

Urbaniak, Wiesław

Urbaniec, Krzysztof

Urbanik, Marek

Urbańska, Weronika Martyna

Urbański, Mariusz

Urbikain, Gorka

Urbina, Antonio

Urbonas, Rolandas

Urlić, Branimir

Uroić, Tessa

Ursu, Ioan

Uruba, Václav

Usaola, Julio

Uscilowska, Anita

Ushakov, Nikolay M.

Ushakov, Sergey V.

Ushida, Akiomi

Ushifusa, Yoshiaki

Uslar, Mathias

Usman, Muhammad

Usmani, Zeba

Ußmüller, Thomas

Ustun, Taha Selim

Utkin, Vadim 
Uykur, Ece

Uzhga-Rebrov, Oleg

Uzunlar, Erdal

Vaagensmith, Bjorn

Vac, Sebastian Călin

Vacca, Andrea

Vaccarini, Massimo

Vaccaro, Luis

Václavíková, Natália

Vadasz, Peter

Vagapov, Yuriy

Vaghasiya, Jayraj

Vagin, Mikhail

Vagiona, Dimitra G.

Vagnoni, Elena

Vahab, Mohammad

Vahdati, Maria

Vahedi, Hani

Vaiana, Nicolò

Vaiciene, Marija

Vaida, Calin

Vaimann, Toomas

Vaisi, Salah

Vajda, Istvan

Vakkilainen, Esa

Vakros, John

Vakulchuk, Roman

Vala, Jiri

Valančius, Kęstutis

Valant, Matjaz

Valderrama Sakuyama, Carlos

Valdez, Jose

Valdiserri, Paolo

Valdmanis, Vivian

Vale, Zita

Valeev, Dmitry

Valencia, Andres

Valencia-Palomo, Guillermo

Valente, Antonio

Valente, Marieta

Valente, Virgilio

Valenti, Francesca

Valentin, Ilea

Valentini, Roberto

Valentino, Roberto

Valenzuela, Miguel

Valerio, Apicella

Valero, Antonio

Valero, Mario M.

Valero, Sergio

Valič, Blaž
Valin, Sylvie

Valipour, Mohammad

Valkealahti, Seppo

Vallejo, Bertha

Valles, Manel

Vallianatos, Filippos

Valls, Pedro

Valogianni, Konstantina

Valouch, Viktor

Valov, Nikolay

Valtchev, Stanimir

Valtierra-Rodriguez, Martin

Valvano, Stefano

Van Belle, Douglas

Van Bommel, Wout

Van Dam, Noah

Van Den Bossche, Alex

Van Den Brom, Helko

Van Der Kam, Mart

Van Der Laan, Paul

Van Der Meer, Theo H.

Van Der Schoor, Tineke

Van Der Weijde, Adriaan Hendrik

Van Dijk, Meine Pieter

Van Driel, Williem Dirk

Van Duijsen, Peter

Van Goeverden, Kees

Van Herk, Alex

Van Hoydonck, Wim

Van Laethem, Dries

Van Loo, Robert

Van Neste, Charles

Van Nguyen, Trung

Van Niekerk, Adriaan

Van Rooijen, Willem Frederik Geert

Van Sark, Wilfried

Van Staden, Raluca

Van Vliet, Oscar

Van Willigenburg, Gerard

Van Wyk, Hans-Werner

Vand, Behrang

Vandevelde, Lieven

Vandevender, John Pace

Vaněk, Jiří

Vaněk, Michal

Van-Hai, Bui

Vanneste, Johan

Vapnik, Yevgeny

Vaqueiro Contreras, Michelle

Varadwaj, Pradeep R.

Varady, Robert 
Varbanov, Petar

Vardakas, John S.

Vardanyan, Yelena

Vardasca, Ricardo

Vardelle, Armelle

Varfolomeev, Mikhail A.

Varfolomejeva, Renata

Varga, Bogdan

Varin, Robert A.

Variny, Miroslav

Varlam, Mihai

Varma, Rajender S.

Varma, Vijaykumar

Varotsos, Costas

Vasandani, Paresh

Vasar, Cristian

Vásárhelyi, Balázs

Vaschetto, Silvio

Vasco-Correa, Juliana

Vasconcelos, Helena Cristina

Vasilateanu, Andrei

Vasilevich, A. V.

Vasiliev, Alexander

Vasiliev, Leonard

Vasiliev, Mikhail

Vasilievici, Gabriel

Vasiljević, Goran

Vasilyev, Andrey

Vasmara, Ciro

Vasquez Padilla, Ricardo

Vassalini, Irene

Vassilev, Igor

Vassiliades, Constantinos

Vasudevan, Suraj

Vâtcă, Sorin

Vathy-Fogarassy, Ágnes

Vatin, Nikolai

Vaverková, Magdalena Daria

Vavrek, Roman

Vaz, Warren

Vázquez Hurtado, Federico

Vázquez, Javier

Vázquez, Manuel

Vedarajan, Raman

Veeneman, Wijnand

Veerakumar, Pitchaimani

Vega, Almudena

Vega-Fuentes, Eduardo

Veintimilla, Salvador García-Ayllón

Veje, Christian
Velamati, Ratna Kishore

Velasco Vélez, Juan J.

Velazquez, Luis

Velazquez-Perez, Jesus E.

Velichkova, Rositsa

Velkavrh, Igor

Velusamy, Gandhimathi

Vencloviene, Jone

Venkatashamy Reddy, Mogalahalli

Venkatesh

Venkiteswaran, Vinod Kumar

Ventouras, Erricos-Chaim

Ventura, Cristina

Ventura, Joao Oliveira

Venturini, Osvaldo José

Venugopal, Chitra

Venuturumilli, Sriharsha

Vepsäläinen, Jari

Vera, David

Verbeeck, Griet

Verbelen, Florian

Verbois, Hadrien

Verde, Francesco

Verdejo Espinosa, María Ángeles

Verga, Francesca

Vergalli, Sergio

Vergura, Silvano

Verhelst, Sebastian

Verma, Amit

Verma, Amrit

Verma, Santosh Kumar

Vermeşan, Horațiu

Verna, Alessio

Vernardou, Dimitra

Vernon, Hodge

Versaci, Mario

Vershinina, Kseniya

Ververidis, Dimitrios

Vescovi, Dalila

Vesnaver, Aldo

Vespasiano, Giovanni

Veszteg, Róbert Ferenc

Veuger, Jan

Via, Brain

Viana, David Leite

Viatkin, Aleksandr

Vibhute, Sandip M.

Viccione, Giacomo

Vicencio, Rodrigo Barraza

Vicente, David J.

Vicente, Paula 
Vicente, Rodriguez Montequin

Vicente, Sandoval

Vicente, Yolanda Sánchez

Vicinanza, Diego

Vidal Vazquez, Francisco

Vidal, Yolanda

Vidal-Albalate, Ricardo

Vidhi, Rachana

Vido, Lionel

Vidović, Danko

Viegas, João Carlos

Vieira, Abel

Vieira, Mário

Vienažindienè, Milita

Viere, Tobias

Viganò, Daniele

Viggiano, Annarita

Vignati, Michele

Vijay, Avinash

Vikulova, Mariya Aleksandrovna

Vila, Carlos

Vilaca, Philipe

Vilanova, Ramon

Vilău, Radu

Vilcekova, Silvia

Vilhelm, Jan

Vilke, Siniša

Villa, Andrea

Villa, Luiz Fernando Lavado

Villa-Arrieta, Manuel

Villacampa Naverac, María Belén

Villacorta, Byron

Villadangos, Jesus

Villalta, Ismael Vallejo

Villani, Manfredi

Villante, Carlo

Villarino, Alberto

Villasmil, Willy

Villaverde, Miguel Ángel Naya

Villeneuve-Faure, Christina

Vilsen, Soren

Vinagre, Eugénia

Vinatoru, Mircea

Vinayagam, Arangarajan

Vincent-Bonnieu, Sebastien

Vincenzi, Donato

Vincenzo, Dovì

Vinh, Nguyen Quang

Vinnik, Denis

Vinod, Ashwin

Vinokurov, Sergey
Vinokurov, Vladimir

Vinothkannan, Mohanraj

Vinuesa, Ricardo

Vio, Gareth

Viola, Fabio

Violano, Antonella

Virag, Zdravko

Virgala, Ivan

Virgone, Joseph

Virtič, Peter

Virtuani, Alessandro

Viscito, Luca

Visconti, Paolo

Visvizi, Anna

Viswanathan, Harish

Viswanathan, Vimal

Viswanathan, Vish

Vita, Vasiliki

Vital, Joaquim Silvério Marques

Vitale, Gianpaolo

Vitázek, Ivan

Vitiello, Silvia

Vítor, Geraldes

Vítová, Milada

Viveiros, Carla

Viviani, Massimo

Vivo, Paola

Vivoda, Vlado

Vižintin, Goran

Vizza, Pasquale

Vlachokostas, Alex

Vlachokostas, Christos

Vlachopoulos, Nick

Vlachos, Nicholas

Vlad, Ciprian

Vladescu, Marian

Vladimir, Kindra

Vladimir, Nikola

Vlahakis, Eleftherios

Vlahinic, Sasa

Vlase, Sorin

Vo, Minh

Vo, Truong

Vocale, Pamela

Vodnar, Dan C.

Voglitsis, Dionisios

Vogtländer, Joost

Voica, Marian Catalin

Voicu, Gheorghe

Voinov, Alexey

Voit, Klaus 
Vojtovic, Sergej

Volat, Christophe

Volkov, Konstantin

Volkova, Anna

Volkova, Luba

Vollbrecht, Joachim

Vollmer, Ina

Volochaev, Vadim A.

Volosencu, Constantin

Volovlikova, Olga

Volpe, Maurizio

Volpe, Richard James

Volpe, Rosaria

Von Cossel, Moritz

Von Gratowski, Svetlana

Von Jouanne, Annette

Von Roon, Serafin

Von Tüllenburg, Ferdinand

Vona, Marco

Vora, Ankit

Vorobieff, Peter V.

Vorobiev, Pavel

Voronenkov, Vladislav

Voropai, Nikolai

Voß, Ursula

Voudouri, Antigoni P.

Vouros, Stavrous

Vovk, Ruslan

Vovos, Panagis

Voyer, Damien

Voz, Cristobal

Vozikis, Dimitrios

Vrålstad, Torbjørn

Vranayová, Zuzana

Vrančić, Damir

$\mathrm{Vu}$, Anh D.

Vucinic, Dean

Vujacic, Marija

Vujević, Slavko

Vujkovic, Milica

Vujović, Igor

Vukobratović, Marko

Vuković Domanovac, Marija

Vukovic, Vladimir

Vuletic, Dijana

Vulic, Milivoj

Vulin, Domagoj

Vun Jack, Chin

Vuong, Quan-Hoang

Vusic, Dinko

Vuta, Liana Ioana
Vyas, Shruti

Vyazovkin, Sergey

Vycital, Vaclav

Vyšvařil, Martin

Wa Li, Danny Hin

Wachowski, Sebastian

Wackernagel, Mathis

Wacławek, Stanisław

Wade, Matthew

Wadrzyk, Mariusz

Waffenschmidt, Eberhard

Wagh, Priyesh A.

Wagner, Beatrice

Wagner, Michael

Wagner, Natalia

Wagner, Norman

Wahed, Arifeen

Waibel, Christoph

Wai-Keung, Fung

Waindok, Andrzej

Wajs, Jan

Walczak, Krzysztof

Walczak, Paweł

Wald, Frantisek

Walendziuk, Wojciech

Walentek, Andrzej

Walheer, Barnabé

Walker, Andy

Walker, Iain

Walker, Ross M.

Walker, Sara Louise

Wallberg, Ola

Wallen, Scott

Walnum, Hans Jakob

Walter, Arnaldo

Waluś, Konrad

Wan, Gang

Wanat, Leszek

Wang, Baicun

Wang, Bing

Wang, Can

Wang, Chaofeng

Wang, Chen

Wang, Cheng-Yu

Wang, Chi-Chuan

Wang, Chih-Liang

Wang, Chuanlong

Wang, Danhong

Wang, Danling

Wang, Enhua

Wang, Fan 


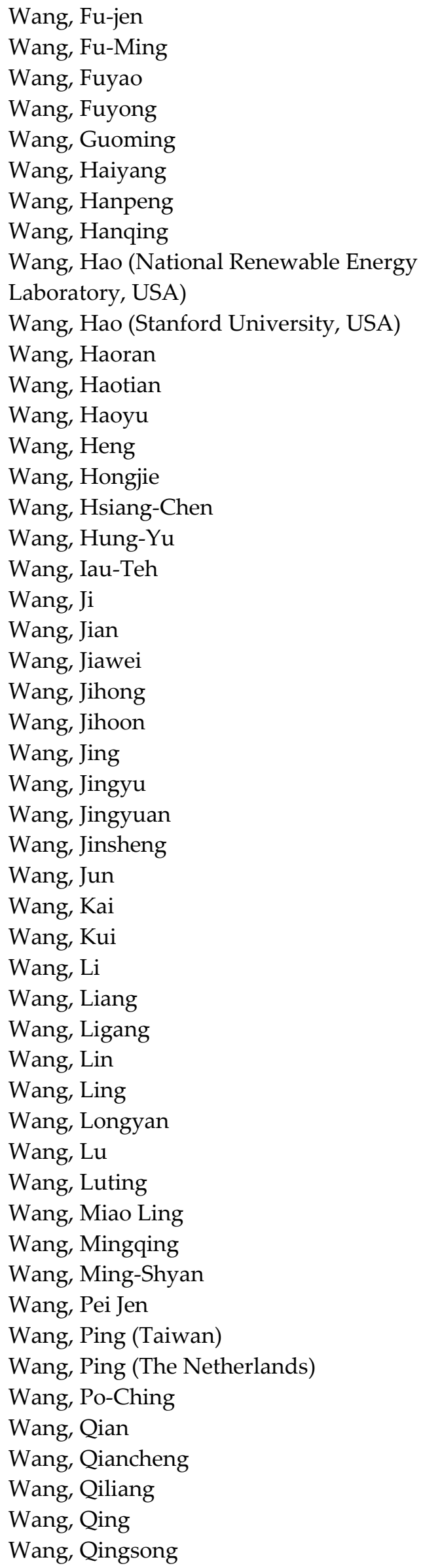

Wang, Qiugu

Wang, Renjie

Wang, Ruixue

Wang, Ruofan

Wang, Sanmin

Wang, Sheng

Wang, Shen-Tsu

Wang, Shiyuan

Wang, Shu

Wang, Shuangyi

Wang, Shuoqi

Wang, Shuoshi

Wang, Sujing

Wang, Taiping

Wang, Teng

Wang, Wei (Australia)

Wang, Wei (China)

Wang, Wei (USA)

Wang, Weizhi

Wang, Xiaoen

Wang, Xiaolin (Australia)

Wang, Xiaolin (USA)

Wang, Xiaoyong

Wang, Xilin

Wang, Xinlin

Wang, Xinyan

Wang, Xiuling

Wang, Xizu

Wang, Xuan

Wang, Xueju

Wang, Xuezhi

Wang, Yanbo

Wang, Yaw-Juen

Wang, Yih-Wen

Wang, Yinzhi

Wang, Yishen

Wang, Youjiang

Wang, Yu

Wang, Yun

Wang, Yun-Che

Wang, Zhanle

Wang, Zhenyu

Wang, Zhipeng

Wanjiku, John

Wanke, Peter

Wanniarachchi, Ayal

Wanniarachchige, Pabasara

Warczek, Jan

Wardach, Marcin

Wardal, Witold Jan 
Warmann, Emily

Warzecha, Adam

Washizu, Ayu

Wasiak, Andrzej

Wasilczuk, Michał

Watanabe, Daisuke

Watanabe, Norihiro

Watanabe, Tsunemi

Watanabe, Yasumasa

Watkins, Kenton Bradley

Wątorek, Marcin

Watrobski, Jaroslaw

Watson, Neville

Watt, Simon

Watter, Holger

Wawro, Aleksandra

Wawrzyniak-Guz, Kamila

Ważny, Mariusz

Wcislik, Miroslaw

Wciślik, Sylwia

Weatherley, Dion

Webborn, Ellen

Weber, Christoph

Weber, Jürgen

Weber, Klaus

Weber, Konradin

Weber, Ludger

Weeks, Brandon

Wegiel, Tomasz

Węglarski, Mariusz

Węglarz, Magdalena

Wehrle, Erich

Wei, An-Chi

Wei, Bo

Wei, Chih-Chiang

Wei, Mingzhen

Wei, Ning

Wei, Tiwei

Wei, Wei

Wei, Zhongbao

Weidlich, Ingo

Weier, Tom

Weindl, Christian

Weintrit, Adam

Weiss, Karl-anders

Wen, Chuang

Wen, Guanghui

Wen, Jialong

Weng, Lei

Wen-Jeng, Ho

Wenk, Hans-Rudolf
Werhahn, Olav

Werle, Peter

Werner, Tim

Wernik, Jacek

Wessel, Ryan

Westerdick, Stephan

Westerman, Wim

Wetula, Andrzej

Whaley, David

Wheeler, Keaton A.

White, Martin

Whitehill, Andrew

Whiteley, Michael

White-Sanchez, Juan

Whitman, Christopher J.

Whitman, Lawrence

Wiatkowski, Mirosław

Wiatowski, Marian

Wichliński, Michał

Wiciak-Pikuła, Martyna

Widen, Joakim

Widera, Barbara

Widera, Marek

Widger, Phillip

Widiatmojo, Arif

Widl, Edmund

Widziewicz-Rzońca, Kamila

Wieczorek, Łukasz

Wiedermann, Alexander

Wielgosiński, Grzegorz

Wierach, Peter

Wierling, August

Wierzbicki, Damian

Wierzbicki, Sławomir

Wigger, Henning

Wiktorski, Tomasz

Wilberforce, Tabbi

Wilcoxon, Ross

Wild, Jochen

Wildi, Walter

Wilhelm, Erik

Wilk, Andrzej

Wilk, Joanna

Wilkosz, Kazimierz

Willems, Bert

Williams, Alan

Williams, Arthur

Williams, Nathan

Willich, Caroline

Willis, Katharine S.

Wilson, Thomas H. 
Winczek, Jerzy

Winiarski, Tomasz

Winjobi, Olumide

Winkler, Hartmut

Winter, Jennie

Winterstein, Franziska

Wiora, Józef

Wirl, Franz

Wiśniewska, Agnieszka

Wiśniewski, Paweł

WISZNIEWSKI, Andrzej

Witczak, Marcin

Witczak, Piotr

Witkowski, Artur

Witonska, Izabela

Witos, Franciszek

Witos, Miroslaw

Witte, Henk

Wladyslaw, Mitianiec

Włodarczyk, Aneta

Wlodarczyk, Barbara

Włodarczyk, Paweł P.

Włodarczyk, Renata

Włodek, Tomasz

Wöhrer, Alexander

Wojciech, Giernacki

Wojciechowski, Jerzy

Wojciechowski, Kamil

Wojciechowski, Rafal M.

Wójcik, Janusz

Wójcik, Marta

Wójcik-Tabol, Patrycja

Wojnárovits, Laszlo

Wojnarowska, Magdalena

Wojnarowski, Paweł

Wojs, Marcin K.

Wojtaszek, Henryk

Wojtaszek, Małgorzata

Wojtkowski, Wojciech

Wójtowicz, Ryszard

Wolak, Artur

Wołek, Marcin

Wolf, Christoph

Wolf, Robert C.

Wolniak, Radoslaw

Wolny, Ada

Wolny, Lidia

Wolny, Stefan

Wołosz, Krzysztof J.

Wołoszyn, Jerzy
Wołowicz, Marcin

Wolsink, Maarten

Won, Myounggyu

Won, Seunggun

Wong, Bryan

Wong, Danny K. Y.

Wong, David

Wong, Ka Kan

Wong, Ling-tim

Wong, Voon-Loong

Wongkasem, Nantakan

Woo, Chang Gyu

Woo, Heesung

Woo, Seong Tak

Woo, Wai Lok

Wood, David

Wood, Joe

Wood, Tony A.

Workie, Bizuneh

Worrall, Stephen

Worwag, Malgorzata

Wosiek, Małgorzata

Wotzka, Daria

Wouters, Peter

Woydt, Mathias

Woźniak, Jacek

Wrenger, Burkhard

Wright, Andrew John

Wróbel, Karol

Wrobel, Luiz

Wróbel, Marek

Wrobel, Rafal

Wrona, Paweł

Wronski, Jorrit

Wrzesiński, Grzegorz

Wu, Bing

$\mathrm{Wu}$, Chienhsun

Wu, Chih-Da John

Wu, Chyan-Chyi

Wu, Deliang

Wu, Di (McGill University, Canada)

Wu, Di (North Dakota State University, USA)

Wu, Di (Pacific Northwest National

Laboratory, USA)

$\mathrm{Wu}$, Haimeng

$\mathrm{Wu}$, Hongwei

$\mathrm{Wu}$, Hui

$\mathrm{Wu}$, Jason (Dayong)

$\mathrm{Wu}$, Jianqing

$\mathrm{Wu}$, Jinn-Chang

Wu, Jiyan 


\begin{tabular}{|c|c|}
\hline Wu, Kai & Xiao, Dequan \\
\hline Wu, Kejun & Xiao, Dunhui \\
\hline Wu, Kuop Jui & Xiao, Jinsheng \\
\hline WU, Lihua & Xiao, Liang \\
\hline Wu, Lixiang & Xiao, Xin (UK) \\
\hline Wu, Ming-Chung & Xiao, Xin (USA) \\
\hline Wu, Nan & Xiao, Xinxin \\
\hline Wu, Pinghui & Xiao, Yang \\
\hline Wu, Qing & Xiao, Zhigang \\
\hline Wu, Qunfang & Xie, Chunping \\
\hline Wu, Rome-Ming & Xie, Jiahan \\
\hline Wu, Shenghua & Xie, Jie \\
\hline Wu, Shih-Jeh & Xie, Jing \\
\hline Wu, Suiwen & Xie, Jingxiu \\
\hline Wu, Wei (Hong Kong) & Xie, Xiaoyan \\
\hline Wu, Wei (Taiwan) & Xie, YuLong \\
\hline Wu, Xiaogang & Xie, Yun \\
\hline Wu, XiaoYu & Xie, Yunchuan \\
\hline Wu, Xinru & Xing, Guichuan \\
\hline Wu, Xuejian & Xing, Lei \\
\hline Wu, Xuezhi & Xing, Yangang \\
\hline Wu, Yonghua & Xiong, Jing \\
\hline $\mathrm{Wu}$, You & Xiros, Nikolaos \\
\hline $\mathrm{Wu}, \mathrm{Yu}$ & $X u$, Bin \\
\hline $\mathrm{Wu}, \mathrm{Yu}-\mathrm{Chi}$ & $X u$, Bo \\
\hline Wu, Yu-En & Xu, Chao (China) \\
\hline Wu, Yujie & Xu, Chao (USA) \\
\hline Wu, Yu-Ting & Xu, Charles \\
\hline Wu, Zhong & Xu, Chungang \\
\hline Wu, Ziping & $\mathrm{Xu}$, Dianguo \\
\hline Wulandana, Rachmadian & Xu, Haowen \\
\hline Wulf, Christina & $\mathrm{Xu}$, Jingyuan \\
\hline Wurz, Marc Christopher & $\mathrm{Xu}$, Jinze \\
\hline Wycisk, Ryszard & Xu, Jiu \\
\hline Wydra, Michal & Xu, Jun \\
\hline Wydra, Sven & Xu, Kaichen \\
\hline Wysocka, Izabela & Xu, Kunning Gabriel \\
\hline Wysocka-Czubaszek, Agnieszka & $\mathrm{Xu}, \mathrm{Li}$ \\
\hline Wyszynski, Miroslaw & $\mathrm{Xu}, \mathrm{Lu}$ \\
\hline Xavier, Ana Maria Rebelo Barreto & Xu, Mingtian \\
\hline Xavier, Pradip & Xu, Pengpeng \\
\hline Xi, Kai & Xu, Shanshan \\
\hline Xia, Guanglin & $\mathrm{Xu}, \mathrm{Tao}$ \\
\hline Xia, Hua & $\mathrm{Xu}$, Tianhua \\
\hline Xia, Shiwei & Xu, Wei \\
\hline Xia, Shunxiang & $\mathrm{Xu}, \mathrm{Xiangguo}$ \\
\hline Xia, Zhenyuan & Xu, Xiangyang \\
\hline Xian, Liang & Xu, Xiaojie \\
\hline Xiang, Ji & $\mathrm{Xu}$, Xuesong \\
\hline Xiang, Lin & $\mathrm{Xu}$, Yanhe \\
\hline Xiao, Boqi & $\mathrm{Xu}, \mathrm{Zhe}$ \\
\hline
\end{tabular}




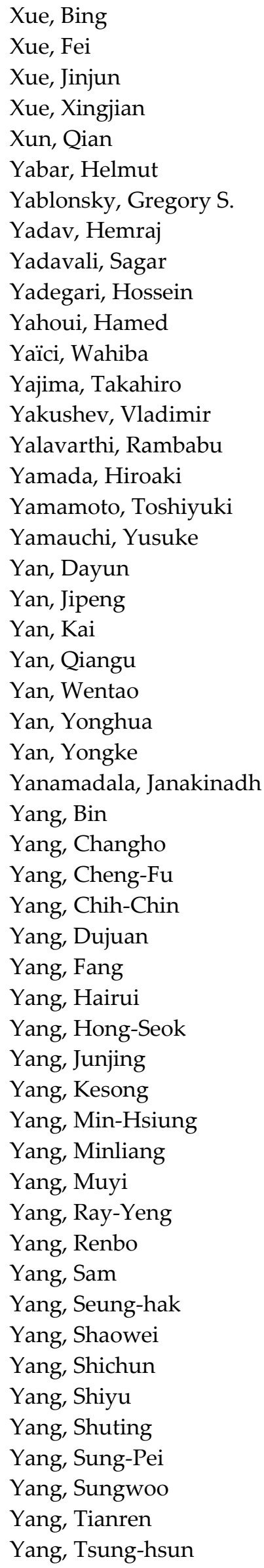

Yang, Wei (Australia)

Yang, Wei (USA)

Yang, Wei-Bin

Yang, Weihong

Yang, Weijia

Yang, Wendong

Yang, Won

Yang, Wuqiang

Yang, Xiaohan

Yang, Xiaolei

Yang, Xiaoyun

Yang, Xinbo

Yang, Xueqing

Yang, Yi (Denmark)

Yang, Yi (USA)

Yang, Yingchen

Yang, Yongheng

Yang, Yoonseok

Yang, Youngoo

Yang, Yu-Chiao

Yang, Yuguang

Yang, Zhaoqing

Yang, Zixu

Yanikara, F. Selin

Yaningsih, Indri

Yannopoulos, Stavros

Yano, Akira

Yano, Mitsuaki

Yao, Bin

Yao, Chunde

Yao, Dingding

Yao, Guolin

Yao, Kai

Yao, Koffi Pierre

Yao, Leehter

Yao, Wenxuan

Yao, Wu-Sung

Yao, Yuchen

Yapici, Murat Kaya

Yaqoob, Raziq

Yasin, Sohail

Yasukawa, Masahiro

Yatchev, Ivan

Yau, Yeu-Torng

Yau, Yung

Yaw, Sean

Yazdani Asrami, Mohammad

Yazdani, Maziar

Yazdani, Morteza

Yazdi, Saeed Varasteh

Ye, Mingxiao 


Ye, Zhi-Ting
Yeh, Chih-Feng
Yelisetti, Subbarao
Yen, Tianming
Yen, Yee-wen
Yentekakis, Ioannis
Yeo, Myoungsouk
Yeom, Chunho
Yermolaev, Oleg Petrovich
Yfoulis, Christos
Yi, Hwang
Yi, Jialiang
Yi, Jin-Hak
Yi, Won-Jae
Yi, Zhehan
Yildirim Yurusen, Nurseda
Yilmaz, Muhittin
Yilmaz, Ozden
Yilmaz, Selin
Yim, Man-Sung
Yim, Yongbin
Yin, Chungen
Yin, Shen
Yin, Wuliang
Yin, Yanting
Yiotis, Andreas
Yitmen, Ibrahim
Yñiguez, Rocío
Yodo, Nita
Yogarathnam, Amirthagunaraj
Yokochi, Alexandre
Yonggang Wen, Wen
Yoo, Chang Geun
Yoo, Kwan-Hee
Yoo, Sungjun
Yoon, En Sup
Yoon, Hwan-Sik
Yoon, Hyun Chul
Yoon, Insun
Yoon, Jeong-won
Yoon, Jeoung S.
Yoon, Keun-Young
Yoon, Kwang S.
Yoon, Minyoung
Yoon, Seong-Min
Yoon, Sungmin
Yörük, Can Rüstü
Yoshida, Kazunari
Yoshihiko, Sano
Yoshimichi, Ohki
Yoshino, Atsushi

Yoshino, Hiroshi

You, Kehua

You, Shutang

You, Tian

You, Young-Hwan

Youn, Jong-Sang

Young, Timothy M.

YoungSoo, Yoon

Yourey, William

Yousef, Samy

Yousefi, Mojtaba

Yousefian, Sajjad

Yousefi-Talouki, Arzhang

Yousif, Charles

Yousif, Jabar H.

Youssef, Ali

Youssef, Tarek

Yrjas, Patrik

$\mathrm{Yu}$, Caoyang

$\mathrm{Yu}$, Chang Wu

Yu, Ding

$\mathrm{Yu}$, Fu-wing

$\mathrm{Yu}$, Guopeng

Yu, Haibo

Yu, Haoran

$\mathrm{Yu}$, Haoshui

Yu, Huai-Te

Yu, Huangchao

$\mathrm{Yu}$, Huapeng

$\mathrm{Yu}$, Ing-Song

$\mathrm{Yu}$, Jaecheul

$\mathrm{Yu}$, Jian

Yu, Lean

Yu, Ruei-Sung

$\mathrm{Yu}$, Ruiyang

$\mathrm{Yu}$, Sangseok

Yu, Tzyy Leon

$\mathrm{Yu}$, Wang

Yu, Wei

$\mathrm{Yu}$, Wenbin

$\mathrm{Yu}, \mathrm{Xi}$

$\mathrm{Yu}$, Xinghuo

$\mathrm{Yu}$, Xinting

$\mathrm{Yu}, \mathrm{Yao}$

$\mathrm{Yu}$, Zhenzhen

Yuan, Haitao

Yuan, Min-Hao

Yuan, Qingcong

Yuan, Quan

Yuan, Tao

Yuan, Titus 


\author{
Yuan, Weijie \\ Yuan, Zhiming \\ Yudintsev, Sergey \\ Yue, Yuan \\ Yuen, Kum Fai \\ Yuldashev, Sh. \\ Yum, Kevin Koosup \\ Yun, Sang-Yun \\ Yurchenko, Daniil \\ Yurkevich, Nataliya V. \\ Yurtsever, Ekim \\ Yurukcu, Mesut \\ Yusuf, Jubair \\ Yuzbasi, Nur Sena \\ Zaballos Diego, Agustín \\ Zabek, Daniel \\ Zabierowski, Wojciech \\ Zabini, Flavio \\ Zabkowski, Tomasz \\ Zabulionis, Darius \\ Zaccaria, Valentina \\ Žáček, Martin \\ Zacharewicz, Gregory \\ Zachariades, Christos \\ Zachariadis, Theodoros \\ Zachariáš, Jiří \\ Zacharof, Myrto-Panagiota \\ Zadorozhnaya, Elena \\ Zafeiratou, Igyso \\ Zafeiriou, Eleni \\ Zaghi, Stefano \\ Zaghib, Karim \\ Zagorowska, Marta \\ Zagorščak, Renato \\ Zagorskas, Jurgis \\ Zagrajek, Krzysztof \\ Zagubień, Adam \\ Zahedmanesh, Arian \\ Zaidan, Martha Arbayani \\ Zając, Grzegorz \\ Zając, Piotr \\ Zajacs, Aleksandrs \\ Zajaczkowski, Bartosz \\ Zajc, Matej \\ Zajkowski, Maciej \\ Zaka Ullah, Malik \\ Zakinyan, Arthur \\ Zakir Abdul Hamid, Umar \\ Zakis, Janis \\ Zambon, Josep B. \\ Zamboni, Giorgio
}

Zambonin, Giuliano

Zambrano, Miller

Zambrano-Martinez, Jorge Luis

Zambroni De Souza, Antônio Carlos

Zamfir, Andreea-Ileana

Zamfir, Rares Halbac-Cotoara

Zamiri, Ali

Zamora, Blas

Zamora, Ramon

Zamparas, Miltiadis

Zamudio-Rivera, Luis S.

Zanchini, Enzo

Zanforlin, Stefania

Zang, Guiyan

Zang, Haixiang

Zangheri, Paolo

Zannis, Theodoros

Zanoletti, Alessandra

Zanon, Bruno

Zapałowicz, Zbigniew

Zapata, Jaime W.

Zapletal, Pavel

Zapp, Petra

Zaragoza, Jordi

Zaragoza, Sonia

Zárate-Miñano, Rafael

Zardin, Barbara

Zare Naghadehi, Masoud

Zarebska, Katarzyna

Zargar, Omid

Zargartalebi, Mohammad

Zarkadoulas, Athanasios

Zarnikau, Jay

Zarraga, Ainhoa

Zarrella, Angelo

Zarzyka, Iwona

Zastawna-Rumin, Anna

Zaton, Marta

Zavadskas, Edmundas Kazimieras

Zavala-Araiza, Daniel

Zavorotynska, Olena

Zawalinska, Katarzyna

Zawieja, Iwona

Zbigniew, Gmyrek

Zbigniew, Leonowicz

Zboinska, Malgorzata A.

Zdankus, Tadas

Zdarta, Jakub

Zdenek Muller, Zdenek

Zdunek-Wiegolaska, Justyna

Zecchin, Roberto 
Zednik, Ricardo

Zehir, Mustafa Alparslan

Zeiler, Wim

Żelazna, Agnieszka

Zemite, Laila

Zemitis, Jurgis

Zempila, Melina-Maria

Zemtsov, Valeriy

Zemtsovskii, Aleksandr V.

Zendehboudi, Sohrab

Zender-Świercz, Ewa

Zeng, Huang

Zeng, Jia

Zeng, Jianwu

Zeng, Wei

Zenger, Kai

Zenith, Federico

Zerefos, Stelios

Zervas, Efthimios

Zetterling, Carl-Mikael

Zewail, Rami

Zeydan, Engin

Zglobicki, Wojciech

Zgraja, Jerzy

Zhai, Shengli

Zhai, Yao

Zhan, Lingwei

Zhan, Meng

Zhan, Siyuan

Zhan, Tianzhuo

Zhan, Xiaowen

Zhang, Alex J.

Zhang, Aron

Zhang, Bo

Zhang, Chaomin

Zhang, Chen

Zhang, Chengbin

Zhang, Cong

Zhang, Dengjun

Zhang, Di

Zhang, Eric J.

Zhang, Feng

Zhang, Fengshou

Zhang, Guannan

Zhang, Gui-Rong

Zhang, Guoyin

Zhang, Hai

Zhang, Han

Zhang, Hangfeng

Zhang, Haochun

Zhang, Haoran
Zhang, Heng

Zhang, HengYun

Zhang, Hongying

Zhang, Jiachen

Zhang, Jian

Zhang, Jiaxin

Zhang, Jiaying

Zhang, Jie

Zhang, Jintao

Zhang, Jize

Zhang, Junxian

Zhang, Kevin H.

Zhang, Laibao

Zhang, Lei

Zhang, Li

Zhang, Lijun

Zhang, Lin

Zhang, Miao

Zhang, Peng (Korea)

Zhang, Peng (USA)

Zhang, Puyang

Zhang, Qi (China)

Zhang, Qi (USA)

Zhang, Qian

Zhang, Qianqian

Zhang, Rui

Zhang, Sheng

Zhang, Shiqiang

Zhang, Tao

Zhang, Tieling

Zhang, Wei (China)

Zhang, Wei (UK)

Zhang, Weige

Zhang, Weiwen

Zhang, Wenbin

Zhang, Wenjun (Chris)

Zhang, Wennan

Zhang, Xiang

Zhang, Xiaohu

Zhang, Xiaoxiao

Zhang, Xiaoxing

Zhang, Xin (China)

Zhang, Xin (UK)

Zhang, Xing

Zhang, Xingxing

Zhang, Xudong

Zhang, Xuesong

Zhang, Xuewei

Zhang, Xuyang

Zhang, Yahui

Zhang, Yan 
Zhang, Yang

Zhang, Yao

Zhang, Yi

Zhang, Yifeng

Zhang, Yiming

Zhang, Yinyan

Zhang, Youwen

Zhang, $\mathrm{Yu}$

Zhang, Yue

Zhang, Yufei

Zhang, Yun

Zhang, Yunyan

Zhang, Yuzhi

Zhang, Zhedong

Zhang, Zhen

Zhang, Zhenbin

Zhang, Zhien

Zhang, Zijun

Zhang, Ziqian

Zhao, Evan Wenbo

Zhao, Haisen

Zhao, Hui

Zhao, Jialin

Zhao, Jiecheng

Zhao, Jingyi

Zhao, Lianfeng

Zhao, Lurui

Zhao, Mingfei

Zhao, Nan

Zhao, Nana

Zhao, Peng

Zhao, Q.

Zhao, Qiuying

Zhao, Ruaikai

Zhao, Rui

Zhao, Shen

Zhao, Shiquan

Zhao, Tianqiao

Zhao, Wenchao

Zhao, Xinran

Zhao, Xinxin (Cindy)

Zhao, Xinyu

Zhao, Xudong

Zhao, Yao

Zhao, Zeyu

Zhao, Zhiming

Zhen, Qi

Zhen, Zhao

Zheng, Bo

Zheng, Bocong

Zheng, Dongdong
Zheng, Fei

Zheng, Jun

Zheng, Kun

Zheng, Minzhang

Zheng, Wandong

Zheng, Xiaofeng

Zheng, Xu-Qian

Zheng, Yi

Zheng, Yiteng

Zheng, Zhixue

Zhi Yung, Tay

Zhironkin, Sergey

Zhong, Lexuan

Zhong, Mingjun

Zhong, Xiangyu

Zhong, Xinjue

Zhong, $\mathrm{Yu}$

Zhou, Bin

Zhou, Hui

Zhou, Jiafeng

Zhou, Jingcheng

zhou, Joe

Zhou, Kaiqing

Zhou, Kan

Zhou, Lei

Zhou, Qun

Zhou, Ran

Zhou, Shan

Zhou, Wenbin

Zhou, Xiang

Zhou, Yaneng

Zhou, Yang

Zhou, Yunlai

Zhou, Yuqing

Zhou, Zhibin

Zhu, Cheng

Zhu, Guoniu

Zhu, Ling

Zhu, Lingxiang

Zhu, Mingming

Zhu, Qilun

Zhu, Qingzi

$\mathrm{Zhu}$, Shan

Zhu, Yanan

Zhu, Ziqiang

Zhuang, Li

Zhuang, Shiqiang

Zhuang, Xiaoying

Zhukov, Victor

Zhuo, Guan-Yu

Zhuo, Yueran 
$\mathrm{Zi}$, Lin

Zi, Yunlong

Zia, Muhammad Fahad

Ziaja, Jan

Zic, Mark

Zicmane, Inga

Zielinska-Dabkowska, Karolina M.

Zieliński, Marcin

Zietkiewicz, Joanna

Zikria, Yousaf Bin

Zilinskas, Antanas

Žilková, Jaroslava

Zima, Krzysztof

Zima, Wieslaw

Zimmerman, Robert $W$.

Zimmerman, William

Zimmermannová, Jarmila

Zimon, Dominik

Zimon, Grzegorz

Zimparov, Ventsislav

Zinovi, Dashevsky

Ziogou, Chrysovalantou

Ziółkowski, Paweł

Ziolo, Magdalena

Ziouche, Katir

Zissis, Georges

Zito, Domenico

Zitti, Gianluca

Zivkovic, Vladimir

Životský, Ondřej

Zizzo, Gaetano

Žlender, Bojan

Zmarzły, Paweł

Zmeureanu, Radu

Zmywaczyk, Janusz

Zobaa, Ahmed

Zoican, Sorin

Zoładek, Maciej

Zöldy, Máté

Zolfaghari, Ashkan

Zoltán, Gabnai

Zoltowska, Izabela
Zong, Yi

Zoppi, Corrado

Zorita-Lamadrid, Angel L.

Zoromba, Mohamed Shafick

Zorpas, Antonis

Zöttl, Gregor

Zou, Changfu

Zou, Liangchao

Zou, Suli

Zou, Xin

Zsembinszki, Gabriel

Zsiborács, Henrik

Zubair, Muhammad

Zuberi, M. Jibran S.

Zubert, Mariusz

Zubitur, Manoli M.

Zubo, Rana

Zucaro, Floriana

Zuccari, Fabrizio

Zucchi, Martina

Zuccolini, Marino Vetuschi

Zuccon, Paolo

Zufferey, Thierry

Zühlsdorf, Benjamin

Zukowski, Miroslaw

Zukowski, Witold

Zúñiga, Andrés A.

Zuo, Wenqiang

Žurovec, David

Žvar Baškovič, Urban

Zverev, Vladimir I.

Zvěřinová, Iva

Zwierzchowski, Ryszard

Zych, Dawid

Życzyńska, Anna

Zydron, Pawel

Zygarlicki, Jarosław

Zygmanowski, Marcin

Żyromski, Andrzej

Zyśk, Janusz

Żywczak, Antoni 